\title{
D-instantons and Effective Couplings in Intersecting D-brane Models
}

Nikolas Akerblom

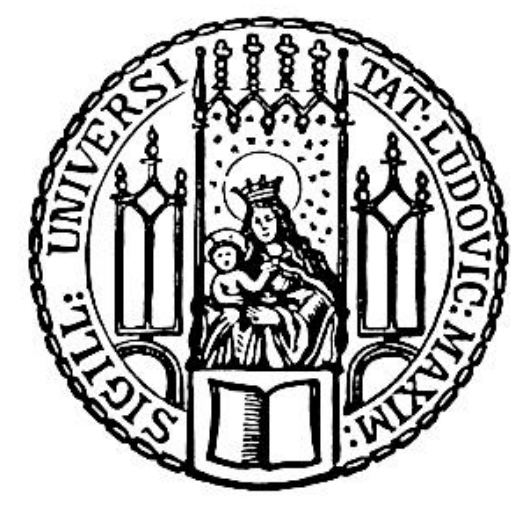

München 2008 



\title{
D-instantons and Effective Couplings in Intersecting D-brane Models
}

Nikolas Akerblom

\author{
Dissertation \\ an der Fakultät für Physik \\ der Ludwig-Maximilians-Universität \\ München
}

vorgelegt von

Nikolas Akerblom

aus Jönköping/Schweden

München, April 2008 
Erstgutachter: PD Dr. Ralph Blumenhagen Zweitgutachter: Prof. Dr. Dieter Lüst Tag der mündlichen Prüfung: 15. Juli 2008 


\section{SECOND Witch}

When the hurlyburly's done,

When the battle's lost and won.

W. Shakespeare, Macbeth, Act 1, Scene 1.

To Heike 



\section{Contents}

\begin{tabular}{ll}
\hline Zusammenfassung & ix
\end{tabular}

Summary $\quad$ xi

\begin{tabular}{lll}
\hline & Introduction and Overview & 1
\end{tabular}

1.1 Why Go Beyond the Standard Model? . . . . . . . . . . . . . . . . . . . . 2

1.2 String Theory . . . . . . . . . . . . . . . . . . . . . . . . . . . . . . . . . . .

$1.2 .1 \quad$ Intersecting D6-brane Models . . . . . . . . . . . . . . . . . . . . . 13

1.2 .2 Discussion . . . . . . . . . . . . . . . . . . . . . . . . . . . . . . . . . . . . . . .

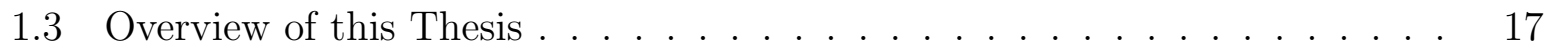

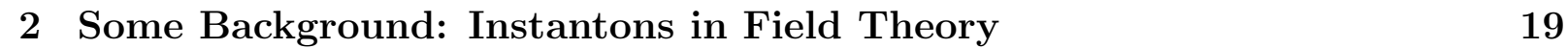

2.1 Introduction . . . . . . . . . . . . . . . . . . . . . . . . . . . . . . . 20

2.2 Instantons in Yang-Mills Theory . . . . . . . . . . . . . . . . . . . . 21

2.3 Instantons in the Euclidean Path Integral . . . . . . . . . . . . . . . . . 25

$2.4 \quad$ Instanton Resolution of the $U(1)$ Problem . . . . . . . . . . . . . . . . . . . 28

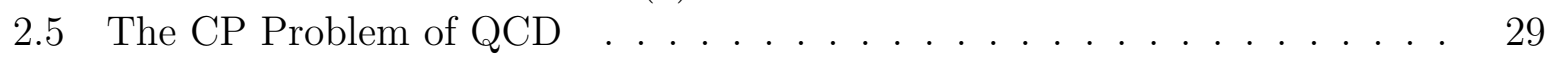

2.6 Baryon and Lepton Number Violations . . . . . . . . . . . . . . . . . . . . . . . . . . . . . . . . . .

2.7 ADHM Construction . . . . . . . . . . . . . . . . . . . . . . . . . . . 32

2.8 The ADS Superpotential . . . . . . . . . . . . . . . . . . . . . . . 33

\begin{tabular}{|lll|}
\hline 3 & The ADS Superpotential from D-instantons & 39
\end{tabular}

3.1 Introduction . . . . . . . . . . . . . . . . . . . . . . . . 40

3.2 Instanton Generated Superpotential . . . . . . . . . . . . . . . . . . . . . . . . . 40

3.3 SQCD from an Intersecting D6-brane Model . . . . . . . . . . . . . . . . . . . . . . . . 44

3.3 .1 Field Theory Limit . . . . . . . . . . . . . . . . . . . . . . . . . 47

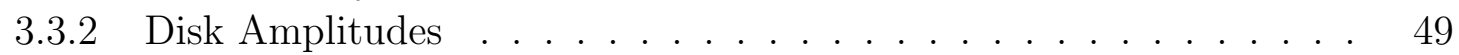

3.4 Calculating the ADS Superpotential. . . . . . . . . . . . . . . . . . . . . . . . . . . . . . . . . . . 53

3.4 .1 Fermionic Zero Mode Integration . . . . . . . . . . . . . . . . . . 53

3.4 .2 Bosonic Zero Mode Integration . . . . . . . . . . . . . . . . . . . . 54

3.5 Relation to Field Theory . . . . . . . . . . . . . . . . . . . . . . . . . . 58 
4 One-loop Corrections to Gauge Kinetic Functions $\quad 61$

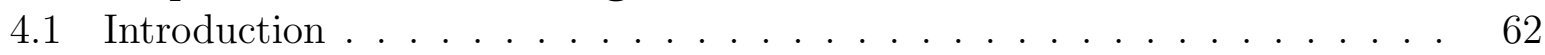

4.2 Non-renormalization Theorems f . . . . . . . . . . . . . . . . . . . 62

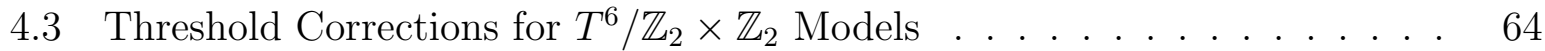

4.3 .1 Background and Calculations . . . . . . . . . . . . . 65

4.4 Extracting $f_{a}^{1-\text { loop }}$ from Gauge Threshold Corrections . . . . . . . . . . . . 77

4.4.1 Results for $T^{6} / \mathbb{Z}_{2} \times \mathbb{Z}_{2}$ Models $\ldots \ldots \ldots$. . . . . . . . 78

\begin{tabular}{|lll}
5 & D-instanton Corrections to Low Energy Effective Couplings & 83
\end{tabular}

5.1 Introduction . . . . . . . . . . . . . . . . . . . 84

5.2 Contributions to the Superpotential . . . . . . . . . . . . . . . . . . 85

5.3 Corrections to Gauge Kinetic Functions . . . . . . . . . . . . . . . . . . . . 89

5.4 Consequences for Toroidal Orbifold Models . . . . . . . . . . . . . . . . . . 90

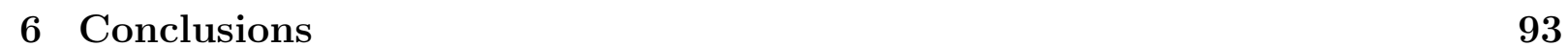

\begin{tabular}{|ll}
\hline A Four Dimensions of Spacetime & 97
\end{tabular}

\begin{tabular}{ll}
\hline B Contributions of Instantons & 103
\end{tabular}

\begin{tabular}{ll}
\hline C Euclidean Field Theory & 105
\end{tabular}

\begin{tabular}{ll}
\hline D Some Relations in Linear Algebra & 109
\end{tabular}

\begin{tabular}{|l|l}
\hline Acknowledgements & 113
\end{tabular}

\begin{tabular}{ll}
\hline Bibliography & 115
\end{tabular} 


\section{Zusammenfassung}

Diese Dissertation beschäftigt sich mit zwei Aspekten von Intersecting D6-brane Modellen: Wir behandeln einige von E2-Instantonen erzeugte Effekte und betrachten Einschleifenkorrekturen zu den eichkinetischen Funktionen in der effektiven Niederenergiefeldtheorie.

Wir beginnen Kapitel 1 mit einer allgemeinen Einführung, in welcher der Frage nachgegangen wird, warum man Stringtheorie als einen Kandidat für eine fundamentale Theorie der Naturgesetze in Betracht ziehen sollte. Hiernach behandeln wir in Kapitel 2 einige Themen, die mit Instantonen in Feldtheorie zusammenhängen und schließen mit dem von Instantonen erzeugten Superpotential der supersymmetrischen QCD, dem sogenannten AffleckDine-Seiberg (ADS) Superpotential.

In Kapitel 3 gehen wir dann daran, das ADS Superpotential in einem Intersecting D6brane Modell als den Effekt eines E2-Instantons herzuleiten. Dazu legen wir zunächst den für die Berechnung des Beitrags eines E2-Instantons zum Superpotential notwendigen Formalismus dar, konstruieren daraufhin eine Realisierung von SQCD in einem (lokalen) Intersecting D6-brane Modell und zeigen schließlich, daß der Formalismus in der Tat das Affleck-Dine-Seiberg Superpotential reproduziert.

In Kapitel 4 betrachten wir Einschleifenkorrekturen zu eichkinetischen Funktionen. Diese Einschleifenkorrekturen hängen in recht komplizierter Art und Weise mit Schwellenkorrekturen zu Eichkopplungen zusammen und wir berechnen diese für $\mathbb{Z}_{2} \times \mathbb{Z}_{2}$ Torus-Orbifold Modelle.

Eine Analyse des Formalismus für die Berechnung des Superpotentialbeitrages eines E2Instantons wirft die Frage auf, ob dieser Beitrag eine holomorphe Größe ist. Wir gehen dieser Frage in Kapitel 5 nach und zeigen, daß der Beitrag in der Tat holomorph ist. Außerdem diskutieren wir, unter welchen Umständen E2-Instanton Korrekturen der eichkinetischen Funktionen zu erwarten sind.

Es schließen sich eine Anzahl von Anhängen an, von denen einige Themen behandeln, die im Haupttext nur gestreift werden und andere notwendige Formeln bringen. 


\section{Summary}

This thesis is concerned with two aspects of intersecting D6-brane models: We treat some of the effects that are induced by E2-instantons and we consider one-loop corrections to the gauge kinetic functions in the low-energy effective field theory.

We begin in chapter 1 with a general introduction covering the question why to consider string theory as a candidate theory of nature. After that, in chapter 2, we go over some topics related to field theory instantons and finally discuss the instanton generated superpotential in supersymmetric QCD (SQCD), the so-called Affleck-Dine-Seiberg (ADS) superpotential.

In chapter 3 we then proceed to reproduce the ADS superpotential in an intersecting D6brane realization of SQCD as the effect of a single E2-instanton. In order to do so, we first explicate the necessary formalism for calculating the E2-instanton contribution to the superpotential, then construct a (local) intersecting D6-brane realization of SQCD, and finally show that the formalism indeed leads to the Affleck-Dine-Seiberg superpotential.

In chapter 4 we consider one-loop corrections to the gauge kinetic functions. These one-loop corrections are related quite intricately to gauge threshold corrections which we compute for intersecting D6-brane models on the $\mathbb{Z}_{2} \times \mathbb{Z}_{2}$ toroidal orbifold.

An analysis of the formulas for calculating the E2-instanton contribution to the superpotential raises the question whether this contribution is a holomorphic quantity. In chapter 5 we take up this question showing that indeed it is holomorphic. In addition, we discuss under which circumstances one can expect E2-instanton corrections to the gauge kinetic functions.

There follow a number of appendices, some elaborating on points touched upon in the main text, some collecting necessary formulas. 
Chapter 1

\section{Introduction and Overview}




\subsection{Why Go Beyond the Standard Model?}

Physics is not a finished logical system. Rather, at any moment it spans a great confusion of ideas, some that survive like folk epics from the heroic periods of the past, and others that arise like utopian novels from our dim premonitions of a future grand synthesis.

Steven Weinberg, Gravitation and Cosmology.

As this is being written, the $S U(3) \times S U(2) \times U(1)$ model of particle physics (patched suitably to take into account neutrino masses), the standard model, accounts for every experimental fact of the subatomic world and it does so with marvelous precision

For instance, its QED sector alone accounts for the anmalous magnetic moment of the electron to nine significant figures. The experimental value preferred by the particle data group [153] is

$$
\left(\frac{g-2}{2}\right)_{\exp }=1159.6521810(7) \times 10^{-6},
$$

which is to be contrasted with the current best theoretical value [14] (to eigth order in the fine structure constant $\alpha$ )

$$
\left(\frac{g-2}{2}\right)_{\text {th }}=1159.65218279(7.71) \times 10^{-6} .
$$

The reason why the QED sector alone can account for the electron's anomalous magnetic moment to such high accuracy is because its mass is comparatively small, so that loop corrections from massive particles are more or less negligible. The situation is different for the muon $\left(m_{\mu} \simeq 200 m_{e}\right)$, making measurements of its magnetic moment a potential laboratory for detecting hitherto unknown particles.

As another success of the standard model (and there are a quasi infinity of them) let us only mention that QCD lattice calculations determine the mass of e.g. the proton to an accuracy of a few percent, given the constituent quark masses [58]. In comparison to the theoretical value of the electron's anomalous magnetic moment, a few percent is, of course, not very precise but given the fact that in the scheme of things the proton mass looks quite random it is nevertheless a remarkable achievement.

However, the bain of fundamental physics has always been that in some places things don't seem to fit. This was so around 1900 where applications of classical statistical mechanics gave nonsensical answers for black-body radiation, culminating in the end in quantum mechanics, and this certainly is so today. The standard model has shortcomings and downright defects.

Some Open Points in the Standard Model. One particular shortcoming of the standard model which comes to mind is, in fact, a gap in our analytic understanding of 
the QCD sector of the standard model. It is currently not possible to understand the confinement of quarks observed in nature in an analytic way, although numerical lattice calculations gives confidence that QCD indeed shows confining behavior (see, for instance, [106]).

Another shortcoming is the arbitrariness of the standard model. The gauge group and particle content is put in by hand, as are the masses of the particles, their representantions, couplings etc. All of these could in principle be just about anything, the only restriction being cancellation of gauge anomalies. In the 19th century such arbitrariness of a physical theory (cf. materials constants) was not considered a problem but nowadays physicists feel confident that large chunks of the world can not only be described but explained. If explanation, then, is the goal, it is a desideratum to further and further reduce the number of parameters in fundamental physical theory.

The list of shortcomings of the standard model clearly could be extended almost indefinitely but let us just leave it at that and mention a few of its defects.

The most conspicuous defect clearly is that the standard model, by design, does not encompass the gravitational interaction. At low energies, leaving out gravity is an excellent approximation but at very high energies gravity becomes the dominant interaction. Such high energies were presumably reached in the very early universe, thus rendering the standard model inapplicable there. Unfortunately, it is not straightforward to include gravity in the standard model. It is, of course, possible to adjoin Einstein gravity to the standard model as a non-renormalizable effective field theory [74, 62, 63, 64, 137, 68. However, since the gravitational sector is non-renormalizable, this theory will not be applicable at high energies, which is the regime we were interested in in the first place.

Even if one questions the practical necessity of formulating a quantum theory of gravitation, for reasons touching on the logical consistency of physics it appears to be absolutely necessary. It is not possible to have one part of physics obey quantum mechanical laws while another part strictly follows classical laws, for this would presumably allow to violate the uncertainty principle. What we thus need, is a quantum theory which in suitable limits reduces to the standard model (particle physics) or general relativity (gravity).

Perhaps related to the fact that the standard model does not know about gravity is the cosmological constant problem, arguably the biggest problem in fundamental physics today. To describe it, let us digress slightly to give a quick overview of the standard (or concordance) model of cosmology.

The Standard Model of Cosmology $\left.\right|^{1}$ The standard model of cosmology takes as its basic principles:

1. The validity of general relativity, including Einstein's equation $R_{\mu \nu}-\frac{1}{2} R g_{\mu \nu}-\Lambda g_{\mu \nu}=$

\footnotetext{
${ }^{1}$ For an elegant and easily accessible overview of cosmology see [112].
} 


\begin{tabular}{l|c|c|c} 
& Radiation & Matter & Vacuum Energy $(\Lambda)$ \\
\hline$p=w \rho$ & $p=\frac{1}{3} \rho$ & $p=0$ & $p=-\rho$
\end{tabular}

Table 1.1: Equations of state for the 'matter' in the universe.

$8 \pi G T_{\mu \nu}$, at large distance scales.

2. Homogeneity and isotropy of the universe's 'matter ${ }^{2}$ distribution on large scales (an assumption confirmed by observation).

The second principle allows one to model the 'stuff' in the universe as perfect fluids, i.e. by energy momentum tensors of the form

$$
T_{00}=\rho, \quad T_{i j}=p g_{i j},
$$

with equation of state

$$
p=w \rho .
$$

It turns out to be an experimental fact that the 'matter' in the universe at large can be modeled by just three types of energy momentum tensors: matter, radiation, and vacuum energy. The corresponding equations of state are summarized in table 1.1. The total energy momentum tensor of all the 'matter' in the universe then is (for convenience we move the cosmological term to the right hand side of Einstein's equation)

$$
T_{\mu \nu}=T_{\mu \nu}^{\text {matter }}+T_{\mu \nu}^{\text {radiation }}+T_{\mu \nu}^{\Lambda} .
$$

From the second principle also follows that the metric describing the spacetime of the universe takes the general form

$$
d s^{2}=-d t^{2}+a^{2}(t) d \Sigma_{k}^{2},
$$

with $a(t)$ some function, called the scale-factor, and $d \Sigma_{k}^{2}$ the metric of any maximally symmetric three-dimensional space (barring topological distinctions there are three of them, and $k=-1,0,1$ accordingly).

Invoking the first principle allows to determine the scale-factor $a(t)$ given the spatial curvature $k$ and the amount of the various types of 'matter' in the universe. The parameters for the current epoch of the FRW model for our universe are taken from observation and are given in table 1.2. A glance at that table tells us that the radiation content of the universe is negligible, the matter ${ }^{3}$ content is roughly $30 \%$ and the bulk of the universe, i.e.

\footnotetext{
${ }^{2}$ In the nomenclature of general relativity, 'matter' (without the quotes) denotes everything which contributes to the right hand side of Einstein's equation.

${ }^{3}$ The term matter here is slightly deceptive, as matter known to us (composed of the known quarks and leptons) only gives $\Omega_{\text {baryon }}=0.044 \pm 0.003$, leaving us with the situation that most of the matter in the universe is unkown to us. This unknown matter is referred to as dark matter. Current suggestions for its interpretation include light superpartners in supersymmetric extensions of the standard model and also axions - particles proposed to solve the $\theta$-problem of QCD (cf. section 2.5).
} 


\begin{tabular}{c|c|c|c} 
Spatial Curvature $k$ & $\Omega_{\text {matter }}$ & $\Omega_{\text {radiation }}$ & $\Omega_{\Lambda}$ \\
\hline 0 & $0.258 \pm 0.030$ & $\sim 10^{-4}$ & $0.742 \pm 0.030$
\end{tabular}

Table 1.2: Favored FRW parameters for the present epoch of our universe [131]. The density parameters $\Omega_{i}$ are defined as $\Omega_{i}=\rho_{i} / \rho_{c}$, where the $\rho_{i}$ are the energy densities of the various 'matter' components, and $\rho_{c}=3 H^{2} M_{P}^{2}$ with $H$ the Hubble parameter and $M_{P}$ the Planck mass.

$70 \%$, is made up of vacuum energy, whose density can be summarized in the (in-)famous cosmological constant [131]

$$
\Lambda \simeq 10^{-123}
$$

in Planckian units.

It turns out that the standard model of cosmology (concordance model, $\Lambda$ CDM model), with its parameters experimentally fixed, is a rather succesful model of the universe. In particular, it reproduces Hubble's law, it explains the relative abundance of light elements and the big bang part of the mode 4 is a quite compelling explanation for the 2.7 Kelvin microwave background radiation permeating the universe. The model does have some potential problems. Many of them (horizon problem, flatness problem, ... ) have a solution in assuming a brief inflationary phase of the early universe but the so-called cosmological constant problem cannot be fixed in this way:

The Cosmological Constant Problem. Since $\Lambda$ is interpreted as the energy density of empty space, it ought to have an interpretation in terms of microscopic physics, i.e. quantum field theory or perhaps string theory. We feel it's safe to say that to date no such explanation exists (cf. [43]). Typically, all attempts at providing a microscopic explanation either give zero or something much to large for the cosmological constant. This is referred to as the cosmological constant problem and it is a notoriously difficult problem. The inability of the standard model (of particle physics) to account for the cosmological constant can clearly be traced back to its ignorance of gravity: vacuum energy in ordinary field theory is physically meaningless, as only energy differences matter there. In a way, this only reinforces the necessity of formulating a quantum theory of gravity $5^{5}$

Since there exist anthropic arguments suggesting the value [146]

$$
\Lambda \simeq 10^{-120}
$$

and there are no good ideas for mechanisms fixing the cosmological constant to the observed value, some scientists have proposed that $\Lambda$ has no microscopic explanation at all but is somehow anthropically selected for.

\footnotetext{
${ }^{4}$ The dense and hot phase of the universe at early times.

${ }^{5}$ Let us hasten to add, even though this means jumping ahead of the story, that string theory, purportedly a quantum theory of gravity, currently does not offer a microscopic explanation of the value of the cosmological constant 122 .
} 
If one rejects anthropic reasoning, then the inability of the standard model of particle physics to predict the value of $\Lambda$ clearly has to be counted as a defect.

Our aim in this section has been to point out that, although the standard model of particle physics is very successful, there clearly are reasons to go beyond it (and we have selected only a few such reasons). Some such reasons come from aesthetic judgement (arbitrariness), while others come from gaps in our ability to explain observations (cosmological constant problem; also the smallness of the QCD $\theta$-parameter comes to mind).

Now, there are many suggestions for theories beyond the standard model. Grand unified theories try to unify, so to speak, the standard model's gauge group, supersymmetric extensions, such as the MSSM, address the hierarchy problem (which we have omitted from our discussion) and string theory which we shall now outline, aims at providing a completely unified description of all interactions, including gravity.

\subsection{String Theory}

We cannot possibly give a complete or even self-contained introduction to string theory here. For this, we refer to any and all of the books [80, 81, 111, 118, 119, 92, 90, 133, 154, [22, 65, 99] on which we have drawn extensively here. Also, the review [105] has proven to be useful in the preparation of this section. The basic idea of string theory, however, is explained quite easily: The fundamental constituents of the world are not zero-dimensional point particles, but tiny one-dimensional objects. Hence the name string theory.

The rudiments of this theory then might go like this (we are following here the sketch given in [151]): A point particle moving in Minkowksi space is described by giving its coordinates $X^{\mu}$ as functions of some parameter $\tau$ (which we can think of as proper time measured from some event). A possible action for this particle then is

$$
S=-\frac{1}{2} \int d \tau \eta_{\mu \nu} \frac{d X^{\mu}}{d \tau} \frac{d X^{\nu}}{d \tau}
$$

If the particle is massless, we supplement this by the constraint $\left(\partial_{\tau} X\right)^{2}+1=0$, saying that the particle moves at the speed of light. A geometrical way to think about (1.9) is to interpret it as the length of the particle's world-line in Minkowski space. Now, a one-dimensional string will trace out a two-dimensional surface in Minkowski space ${ }^{6}$ The coordinates of the string therefore depend on two parameters: $X^{\mu}=X^{\mu}\left(\sigma^{\alpha}\right)$, with

\footnotetext{
${ }^{6}$ If the string is a little loop, a closed string, it will trace out a tube. If the string is open, it will trace out a surface which does not close on itself. In both cases, one speaks of the world-sheet of the string.
} 
$\sigma^{\alpha}=(\sigma, \tau)$. We take as its action the following obvious generalization of 1.9

$$
S=-\frac{1}{2 \alpha^{\prime}} \int d \sigma d \tau \eta_{\mu \nu} \frac{\partial X^{\mu}}{\partial \sigma^{\alpha}} \frac{\partial X^{\nu}}{\partial \sigma^{\alpha}}
$$

We take the string to be massless and thus, as in the point particle case, we have to impose some constraints. They are $\left(\partial_{\tau} X \pm \partial_{\sigma} X\right)^{2}=0.7$ We have also introduced a constant $\alpha^{\prime}$, called the Regge slope. To make the action come out with the right dimension, we take this constant to have dimension (length) ${ }^{2}$. The theory we have arrived at is technically known as the bosonic string in conformal gauge. When the theory is manipulated according to the rules of some suitable quantization prescription and 'patched' to take into account interactions of strings by splitting and joining, one finds that: (1) the theory requires 26 dimensions of spacetime for its consistency, (2) the spectrum of quantized excitations of the string contains a massless spin-two particle which can be interpreted as the graviton, the supposed exchange quantum of the gravitational field, and (3) the theory incorporates gauge-type interactions. Point (1) calls for some way of making the unwanted extra 22 dimensions small, and we will comment on compactification later on. The other points, however, do look encouraging. Unfortunately, the bosonic string further: (A) contains a particle of negative mass (a tachyon), and (B) has no fermionic particles amongst its excitations. Of these two points (A) is undesirable, but (B) is a disaster, rendering the bosonic string theory a toy-model.

A way to keep the good features of the bosonic string theory and also to have fermions in the spectrum is to modify the field theory in two-dimensions represented by 1.10 . One adds additional anticommuting degrees of freedom $\psi^{\mu}(\sigma, \tau)$, referred to as world-sheet fermions. The action then becomes

$$
S=-\frac{1}{2 \alpha^{\prime}} \int d \sigma d \tau \eta_{\mu \nu}\left(\frac{\partial X^{\mu}}{\partial \sigma^{\alpha}} \frac{\partial X^{\nu}}{\partial \sigma^{\alpha}}-i \bar{\psi}^{\mu} \rho^{\alpha} \frac{\partial}{\partial \sigma^{\alpha}} \psi^{\nu}\right) .
$$

The $\rho^{\alpha}$ are two-dimensional generalizations of the Dirac-matrices and this action too must be supplemented by additional constraints, which we omit here. The two-dimensional field theory defined by $(1.11)$ exhibits $\mathcal{N}=1$ supersymmetry relating the bosonic fields $X^{\mu}$ and the fermionic ones $\psi^{\mu}$, and the closed string theory taking as its starting point (1.11) is referred to as the (type II) superstring. In addition to supersymmetry on the world-sheet, the theory also exhibits $(\mathcal{N}=2)$ supersymmetry in spacetime, that is, after a manipulation known as the GSO projection. It turns out that for the GSO projection one can make two physically distinct choices, one yielding a non-chiral theory called type IIA, the other yielding a chiral theory called type IIB.

The type II theories are not the only supersymmetric string theories in existence. There is also a theory called type I and two so-called heterotic string theories with gauge groups $E_{8} \times E_{8}$ and $S O(32)$. The heterotic theories are theories of closed strings ${ }^{8}$ There are

\footnotetext{
${ }^{7}$ In this overview we do not want to discuss the origin of these Virasoro constraints. For this we refer to the literature mentioned earlier.

${ }^{8}$ Recently, it has been suggested that the $S O(32)$ heterotic string also includes open strings [121].
} 


\begin{tabular}{l||c|c|r} 
Theory & SUSY $\left(\mathcal{N}_{10}\right)$ & Chiral? & Gauge group \\
\hline \hline Type I & 1 & yes & $S O(32)$ \\
Type IIA & 2 & no & none \\
Type IIB & 2 & yes & none \\
Heterotic $S O(32)$ & 1 & yes & $\operatorname{Spin}(32) / \mathbb{Z}_{2}$ \\
Heterotic $E_{8} \times E_{8}$ & 1 & yes & $E_{8} \times E_{8}$
\end{tabular}

Table 1.3: The five superstring theories in ten dimensions. Indicated is the type of supersymmetry in ten dimensions, whether the respective theory is chiral, and its gauge group.

thus five superstring theories but, starting in the mid 1990s, evidence has mounted that these five theories are connected by an intricate web of dualities. This, in turn, has led to speculations that they are all just manifestations of one single theory. A definition of this theory is, as of yet, not known but researchers usually refer to it by the name of M-theory.

Let us describe the five superstring theories in a little more detail. Besides exhibiting spacetime supersymmetry, thus having fermions, all superstring theories require 10 dimensions of spacetime. They do not contain tachyons as the bosonic string does, but they do contain gravity and some exhibit gauge interactions. The massless bosonic spectrum of all five theories also contains the dilaton field $\Phi$ which fixes the string coupling constant $t^{9} g_{s}$ by its vev: $g_{s}=\left\langle e^{\Phi}\right\rangle$. Table 1.3 lists some salient features of the five superstring theories in ten dimensions.

The massless (bosonic and fermionic) excitations of the superstring theories and their interactions are describable by supergravity theories, in some cases coupled to super YangMills theory. This is summarized in table 1.4. In addition to the massless particles which can be described by low energy effective (local) field theories, each string theory also contains infinitely many excitations of arbitrarily high mass and spin. It is remarkable that in string theory it is possible to calculate the interactions of these states, as there appear to be no local quantum field theories describing fields of spin greater than 2 (cf., for instance, 143, section 5.7). From this one can see that string interactions have a non-local character.

Now, the five superstring theories mentioned so far were known already in the mid 1980s, but as mentioned already, in the mid 1990s it became clear that all these theories are in one way or another related to each others by dualities. For instance, the type I theory for $g_{s}$ large (strong coupling) is dual to the heterotic $S O(32)$ theory for small $g_{s}$ and vice versa, the type II theories compactified on a circle are dual to each other upon inversion of the circle's radius (T-duality) and so on and so forth. We will not describe all these dualities here in detail (see [133] for a lucid discussion). However, see figure 1.1 for a visualization of the situation.

\footnotetext{
${ }^{9}$ The string coupling, of course, features in string interactions. See below.
} 


\begin{tabular}{l||l} 
Theory & Low energy effective field theory \\
\hline \hline \multirow{2}{*}{ Type I } & $\begin{array}{l}\mathcal{N}=1 S O(32) \text { super Yang-Mills coupled to } \\
\text { Type I Supergravity }\end{array}$ \\
\hline Type IIA & Type IIA Supergravity \\
\hline Type IIB & Type IIB Supergravity \\
\hline Heterotic $S O(32)$ & $\begin{array}{l}\mathcal{N}=1 S p i n(32) / \mathbb{Z}_{2} \text { super Yang-Mills cou- } \\
\text { pled to Type I Supergravity }\end{array}$ \\
\hline Heterotic $E_{8} \times E_{8}$ & $\begin{array}{l}\mathcal{N}=1 E_{8} \times E_{8} \text { super Yang-Mills coupled to } \\
\text { Type I Supergravity }\end{array}$
\end{tabular}

Table 1.4: A listing of the low energy effective theories for the five superstrings in ten dimensions.

\begin{tabular}{ll}
\hline D $p$-branes & $p$ \\
\hline Type I & $1,5,9$ \\
Type IIA & $0,2,4,6,8$ \\
Type IIB & $-1,1,3,5,7$ \\
\hline
\end{tabular}

Table 1.5: The kinds of stable (BPS) $\mathrm{D} p$-branes present in the five superstring theories. The worldvolume of a $\mathrm{D} p$-brane is a $(p+1)$-dimensional timelike submanifold of spacetime. The $\mathrm{D}(-1)$-brane of type IIB string theory thus has a worldvolume which is a point; it resembles a spacetime instanton in ten dimensions.

An important aspect of these discoveries in the 1990s was the realization that (super-)string theory is not a theory of strings alone but that there also ought to exist a cornucopia of extended objects, to which one refers to collectively as branes 10 One particular class of branes are the so-called D-branes, which are distinguished by the property that they are the objects on which open strings end. Quantum mechanically, not all dimensionalities of D-branes are stable but those that are, are listed in table 1.5. The interest of D-branes for our purposes (see 1.2.1 below) lies in the fact that the massless excitations, i.e. zero modes, of open strings ending on them (typically) make up a supersymmetic gauge theory on their worldvolume.

Before closing this section, let us remark on the general structure of string interactions. The basic idea is that individual strings interact via splitting and joining. In spacetime this means that for, say, three interacting strings there is but a single worldsheet. The idea is illustrated in figure 1.2. For such a string diagram, as it is called, one can then write an amplitude analogous to point particle theories. In figure 1.2 there is only shown the lowest order of perturbation theory for the interaction depicted there. In fact, in perturbative string theory, defined via the Polyakov path integral, there is the instruction to sum over all possible topologies of world-sheets connecting the initial and final string

\footnotetext{
${ }^{10}$ It is true that already in the 1980s it was known that type II string theory contains branes. However, at first they were slightly neglected and only came into the limelight, so to speak, some time later on.
} 


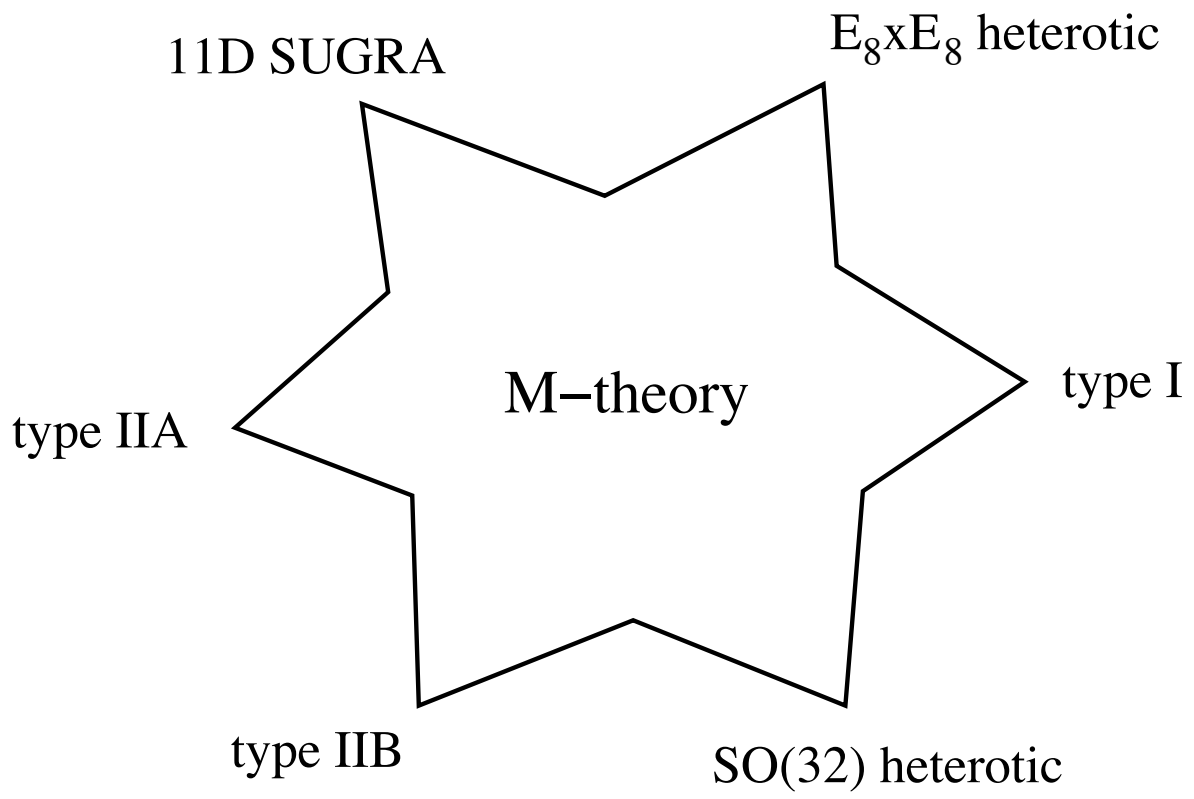

Figure 1.1: All five superstring theories are related by an intricate web of dualities. This fact is taken as an indication that they are all just manifestations of a single underlying theory, tentatively called M-theory. Interestingly, tangled up in this web of dualities is also supergravity in eleven dimensions. Therefore, M-theory will surely not be a theory in strictly ten dimensions - perhaps the notion of 'dimension' will not even be applicable.

configurations. Technically, one has to work with the Euclidean version of the world-sheet theory so that the path integral is, in some sense, defined. All two-dimensional manifolds, i.e. Riemann surfaces, have been topologically classified in the nineteenth century. They can be obtained from the sphere by gluing on handles, cutting out holes, and attaching crosscaps. If one adds crosscaps, the resulting world-sheets are non-orientable and this is the case for, e.g., the type I string theory. For the simpler case of oriented world-sheets, the resulting perturbation theory is easily illustrated, see figure 1.3. With each hole there is associated a factor of $g_{s}^{2}$. Generally, for any scattering amplitude $A\left(g_{s}\right)$ in (perturbative) string theory one has a topological expansion

$$
A\left(g_{s}\right) \propto \sum_{\chi} g^{-\chi} A_{\chi}
$$

where the sum is organized according to the Euler number $\chi$ of each diagram $A_{\chi}$. The crucial point here is that the expansion is in terms of powers of the string coupling constant $g_{s}$. The calculation of any term $A_{\chi}$ in that expansion, in turn, is performed via the methods of conformal field theory and this often proceeds in a perturbative fashion as well, namely order by order in the Regge slope $\alpha^{\prime}$. Now, generally speaking, such a perturbative expansion might not completely capture the observable. First of all, the series usually does not converge, but only is to be interpreted as an asymptotic expansion. Second, observables may have a dependence on the coupling constant not amenable to power series 


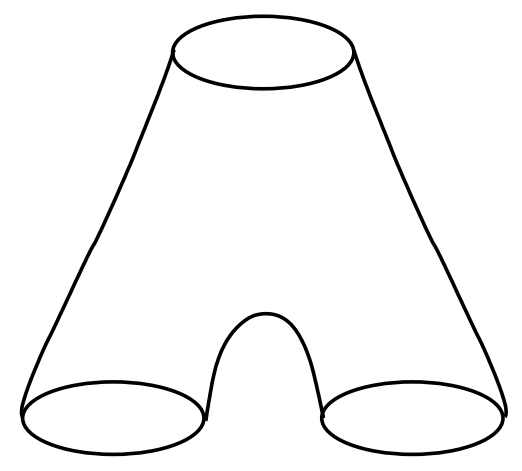

(a)

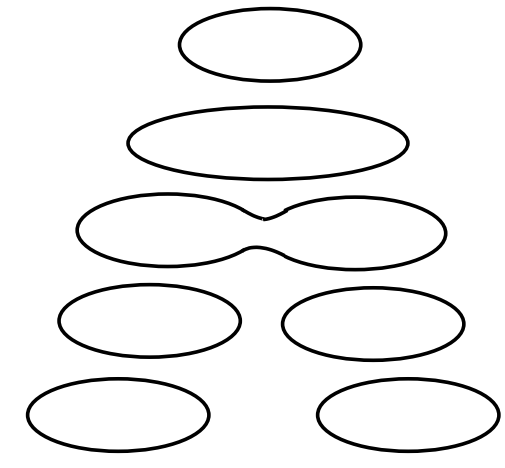

(b)

Figure 1.2: Strings interact via splitting and joining. (a) shows the worldsheet for the joining of two closed strings to form a single closed string. (b) shows the same process at successive instances in time.

expansions. In fact, all instanton effects are of this form! What this means, then, is that one ought to add 'non-perturbative' terms to the perturbative expansion, accounting for instanton contributions, which have to be calculated by some other means. Clearly, there can be such corrections to the expansion in $g_{s}$ [127], called spacetime instanton corrections, but also to the expansion in $\alpha^{\prime}$ mentioned above, called world-sheet instanton corrections [66, 67]. We mentioned earlier that $g_{s}$ is not a parameter but is the vev of some field. This is in line with the general fact that string theory has no dimensionless parameters. Of course, for the application of perturbation theory one then has to assume that $g_{s} \ll 1$.

String Phenomenology. Broadly speaking, string phenomenology refers to that part of research in string theory which directly seeks to make contact between the theory and the observed world. The five superstring theories mentioned in the previous section do have quite remarkable features (all contain gravity, some in addition are chiral and have gauge symmetry). However, as they stand they are clearly unrealistic. The most conspicuous shortcoming is that they are defined in ten-dimensional Minkowski space. In addition, there is also the problem that their low energy particle spectrum is incongruous with what is observed in nature. It is remarkable, that these problems are somehow related. Namely, trying to solve the first problem (too many dimensions) shows the way to solving the other.

Let us go back to (1.11). In that equation there is the metric of Minkowski space $\eta_{\mu \nu}$. It is tempting to try to formulate any of the five superstring theories in spacetimes with a different ('non-trivial') metric and topology. In particular, it appears promising to consider ten-dimensional spacetimes $M$ of the form ${ }^{11}$

$$
M=M_{4} \times K,
$$

\footnotetext{
${ }^{11}$ This is just the simplest ansatz one can make.
} 


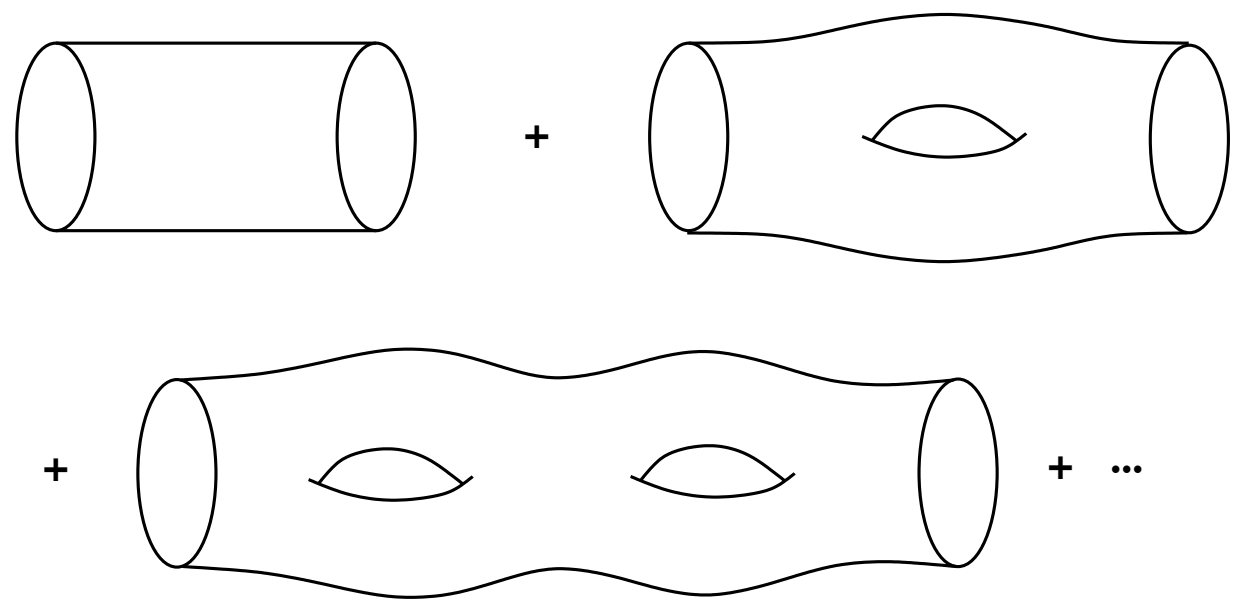

Figure 1.3: Topological expansion for the propagation of a single closed oriented string. Each hole carries a factor of $g_{s}^{2}$.

where $M_{4}$ is four-dimensional Minkowksi-space and $K$ is some six-dimensional compact manifold. This is referred to as compactification. Just why precisely four dimensions should be large we cannot tell, except by appealing to experience or perhaps to anthropic reasoning. This is, of course, quite unfortunate. For the anthropic perspective, see appendix A.

The question then arises what kind of manifold $K$ should be. It turns out that first of all, it should be Ricci flat ${ }^{12}$ The further details depend on the particular string theory one is working with. For the heterotic string, for example, one could require the compactified theory to give $\mathcal{N}=1$ supersymmetry in four dimensions. The remarkable result of this requirement is that $K$ is then restricted to be a so-called Calabi-Yau manifold, which means that it is complex (here, three-dimensional), Kähler, and Ricci flat. There are, of course, also other compactifications involving the various string theories, say on orbifolds (which are not manifolds at all, but can be thought of as singular limits of manifolds), on K3 manifolds and so on and so forth. We obviously cannot survey all known classes of compactifications here. Let us only point out that choosing the background fixes the lower dimensional physics completely, i.e. the type of matter present and its interactions. Thus, in a way, string phenomenology reduces to getting the background right. This is about as difficult as it can get, but over the years remarkable progress has been made. Nevertheless, choosing some background by fiat clearly is an arbitrary act and therefore the hope is shared by some that by a currently unknown dynamical process a particular compactification might be singled out.

\footnotetext{
${ }^{12}$ This requirement is modified if one chooses to include other background fields from the massless spectrum of the respective string theory. Namely, then the Einstein equations (in principle including modifications) with the background fields as sources have to be satisfied. Also, in this case, the simple product ansatz 1.13 perhaps has to be modified. So-called flux compactifications are examples for compactifications with non-trivial background fields.
} 
Now, in the preceding paragraph the words 'compactification' and 'background' can be understood quite broadly to mean also to include background fields different from the metric, and perhaps also D-branes. Including D-branes is especially attractive, as it allows one to construct compactifications giving quite realistic physics in four dimensions in a rather lucid way. In the next section we give a brief discussion of so-called intersecting D6-brane models.

\subsubsection{Intersecting D6-brane Models}

We mentioned above that, under suitable conditions, a supersymmetric gauge theory is induced on the world-volume of a D-brane. This observation is the starting point for string phenomenology with intersecting D-branes. Since we will be working with such particular models later on, we give a brief summary of the general framework here. We will be quite brisk and refer to the review articles [108, 32, 35] and references therein for further information. We also freely cite from these sources here and in later chapters.

For intersecting D6-brane models we are dealing with orientifolds of type IIA superstring theory compactified on a Calabi-Yau threefold $X{ }^{13}$ i.e. the geometric background is of the form

$$
M=\left(M_{4} \times X\right) /(\Omega \bar{\sigma}),
$$

where $M_{4}$ denotes four-dimensional Minkowski space, and the worldsheet parity $\Omega$ acts as $(\sigma, \tau) \mapsto(-\sigma, \tau)$. Further, the antiholomorphic involution $\bar{\sigma}$ in local complex coordinates $z_{i}$ on the Calabi-Yau is often just complex conjugation: ${ }^{14}$

$$
\bar{\sigma}: z_{i} \mapsto \bar{z}_{i}, \quad i=1, \ldots, 3 .
$$

Orientifolding introduces orientifold O6-planes wrapping the fixed locus of the orientifold map which is a union of special Lagrangian three-cycles in the Calabi-Yau. We focus on the case of one orientifold plane and denote its wrapping cycle by $\Pi_{\mathrm{O} 6}$.

We then introduce stacks of D6-branes into this background, where four directions of each D6-brane fills out $M_{4}$ completely. On the internal Calabi-Yau the branes wrap topologically non-trivial special Lagrangian three-cycles $\Pi_{a} \subset X$. Then, their orientifold images will wrap certain three-cycles $\Pi_{a}^{\prime}$. The orientifolding mentioned above, under certain conditions to be discussed, cancels the integrated RR charge and tension introduced by the D6-branes into the Calabi-Yau. Intuitively, this is necessary to satisfy the generalized Gauss' law, as the charge cannot escape to infinity in a compact space. Technically, the orientifolding makes possible RR tadpole cancellation, i.e. the mutual cancellation of the divergences of

\footnotetext{
${ }^{13}$ It is customary to refer to the compactification manifold as a Calabi-Yau, even though it can also be a toroidal orbifold. We will follow this custom quite often.

${ }^{14}$ We have left out a factor of $(-1)^{F_{L}}$, where $F_{L}$ is the left-moving fermion number, as this is of no great importance at the level of the geometric background. However, for the world-sheet theory it is important and we refer to the reviews mentioned earlier for details.
} 


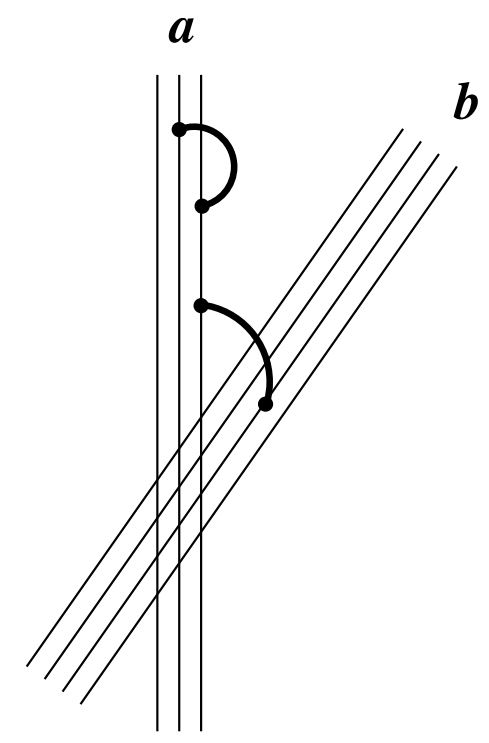

Figure 1.4: In intersecting D6-brane models, matter transforming under (non-)abelian gauge symmetries arises at the intersections of stacks of D6-branes. The strings starting and ending on one and the same stack give gauge bosons and adjoint matter, while the strings starting and ending on different stacks give bi-fundamental, often chiral, matter. In this figure the relevant gauge groups would be $U(3)$ (as $N_{a}=3$ ) and $U(4)$ (as $N_{b}=4$ ).

the Klein bottle, annulus, and Möbius (vacuum) diagrams. For this to happen, the D6branes have to wrap (special Lagrangian) three-cycles in such a way that the homological equation

$$
\sum_{a} N_{a}\left(\Pi_{a}+\Pi_{a}^{\prime}\right)=4 \Pi_{\mathrm{O} 6}
$$

is satisfied, where $N_{a}$ is the number of branes on stack $a$. Since we will only be having to do with supersymmetric configurations, we do not need to elaborate on the further condition of absence of NSNS tadpoles. For supersymmetric configurations it is automatically satisfied.

Gauge bosons in intersecting D6-brane models come from open strings whose end points are attached to the same stack, while (bi-fundamental) matter comes from open strings whose end points are attached to different stacks. For simplicity we consider only the case where all branes wrap cycles not invariant under the antiholomorphic involution. Then the gauge symmetry is $\prod_{a} U\left(N_{a}\right) \cdot{ }^{15}$ In figure 1.4 we show two stacks of branes and the open strings associated with them. In table 1.6 we summarize the chiral matter and the representations of $U(N)$ it tranforms under. In some instances, e.g. in chapter 3 , it is desirable to have nonchiral matter. We will give the particular setup for this in situ. The low energy physics of intersecting D-brane models is (typically) governed by $\mathcal{N}=1$ supergravity. Part of this description are the superpotential for the matter fields and gauge kinetic functions.

\footnotetext{
${ }^{15}$ Dropping the requirement of non-invariance under the antiholomorphic involution, the other possible gauge groups are of the $S O(N)$ and $S p(N)$ type.
} 


\begin{tabular}{l|l} 
Representation & Multiplicity \\
\hline$A_{a}$ & $\frac{1}{2}\left(\Pi_{a}^{\prime} \circ \Pi_{a}+\Pi_{\mathrm{O} 6} \circ \Pi_{a}\right)$ \\
$S_{a}$ & $\frac{1}{2}\left(\Pi_{a}^{\prime} \circ \Pi_{a}-\Pi_{\mathrm{O} 6} \circ \Pi_{a}\right)$ \\
$\left(\bar{N}_{a}, N_{b}\right)$ & $\Pi_{a} \circ \Pi_{b}$ \\
$\left(N_{a}, N_{b}\right)$ & $\Pi_{a}^{\prime} \circ \Pi_{b}$
\end{tabular}

Table 1.6: Chiral spectrum of intersecting D6-brane models. $A_{a}$ denotes the anti-symmetric representation of $U\left(N_{a}\right)$, while $S_{a}$ denotes its symmetric representation. $(\cdot, \cdot)$ denotes the (anti-)bi-fundamental representations of $U(N)$.

The tree-level (in $g_{s}$ ) results for these quantities receive corrections. The gauge kinetic functions receive one-loop perturbative corrections and both can receive non-perturbative corrections. The non-perturbative corrections in $g_{s}$, 'spacetime instanton corrections', come from D-instantons, which, for intersecting D6-brane models, are D2-branes wrapped on three-cycles of the Calabi-Yau. It will be convenient to call these branes E2-instantons. Generally, Ep-instantons have $(p+1)$-dimensional world-volume. Therefore, the a priori candidate Ep-instantons we would have to consider in intersecting D6-brane models would be E0-, E2-, and E4-instantons. However, since there are no topologically non-trivial oneand five-cycles on a Calabi-Yau, the only instantons left are our E2-instantons. Since a D2-brane has three-dimensional world-volume, an E2-instanton necessarily is point-like in four-dimensional Minkowski space. We will come back to E2-instantons in chapters 3 and 5.

\subsubsection{Discussion}

We began this chapter by pointing out some open points in the standard models of particle physics and cosmology taken together. Therefore, we ought to give some kind of assessment whether these problems are addressed in the current understanding of string theory. Our feelings about this are mixed. The answers are all somehow interrelated but let us go through the (select) open points we mentioned and what string theory currently has to say about them one by one.

Analytic Understanding of QCD. In recent years there has been an enormous amount of activity in the so-called AdS/CFT correspondence, holographically relating certain string theories and conformally invariant field theories [6]. This correspondence has been applied with some success to baryonic processes at the RHIC experiment [130]. This in itself is quite remarkable and has led to hopes that perhaps string theory might some day feature as a calculational tool in elucidating the confinement problem of QCD. 
Arbitrariness of the Standard Model. In certain respects, the structure of string theory seems to be remarkably rigid (recall that all known (super-)string theories appear to be limits of a single theory, M-theory). However, this uniqueness is lost to some extent in compactifying down to four dimensions: There are a 'quasi-infinity' of choices for the compactification manifold, branes, fluxes etc. Currently, these choices are driven almost exclusively in a so-called bottom up approach, by the desire to agree with the standard model of particle physics $1{ }^{16}$ It is, of course, possible that there simply is no compelling theoretical explanation that will make the seeming arbitrariness of the standard model understandable and that the background in string theory is determined anthropically [125, 132 . However, this would be a pity judging by initial hopes of explanation from the early days of string theory. Moreover, there is the argument that the background in string theory ought to become a dynamically determined object one day in order to comply with a certain type of spacetime philosophy originating with general relativity. Maybe in such a formulation-to-be of string theory uniqueness will be restored. At least such are the hopes.

Quantum Gravity. Generally speaking, the quantum gravity provided by perturbative string theory is of a type which would be expected in a particle physics context, conceptually almost identical with what one gets when adjoining general relativity to the standard model (cf., for instance, [89]). Namely, the gravitational interaction is mediated by the graviton propagating on a fixed background manifold. A remarkable feature of (perturbative) string theory is that one has a complete description of the graviton's interactions with all the other states of the theory, up to arbitrarily high energies. This definitely is a marvelous improvement over the effective field theory approach to general relativity. There have also been great achievements in the holographic description of quantum gravity and the calculation of black hole entropy, vindicating the results of Bekenstein and Hawking. Still, it is, in our opinion, unfortunate that one is forced to work with a fixed background selected by hand since in this way the deep conceptual problems of quantum gravity are, arguably, sidestepped.

The Cosmological Constant Problem. Possibly related to the problem of the type of quantum gravity provided by string theory is the fact that currently string theory cannot predict the observed smallness of the cosmological constant except by invoking the idea that it is anthropically selected. The invocation of the principle in string theory has been made possible by showing that there are huge number of string theory backgrounds giving metastable ground states with widely varying positive cosmological constant [39, 91]. It is conceivable that this understanding will change over time, perhaps through a better understanding of string cosmology.

\footnotetext{
${ }^{16}$ In fact, the goal often is to agree with the MSSM, as the belief is firm amongst many theorists that this extension of the standard model is bound to be correct.
} 
The above modest assessment of the situation of string theory should make it clear that a lot needs to be done. In particular, constructing ever more realistic phenomenological models of string theory is a major part of current research. This is indeed a worthwhile enterprise as it allows to understand the current formulation of string theory better, leaving aside dreams of a future version. However, the stability and hence consistency of string compactifications surely depends on extensions of the usual perturbative scheme. Here we are thinking of instanton effects. Since these extensions can be made in a more or less controlled manner this seems a good place to extend string theory beyond its perturbative formulation and indeed, instanton effects in string theory have been the subject of intense research since the 1980s (a selection of classic publications is [66, 67, 23, 150, 78, 79, 83, 152, 41, 20, 21]). However, for intersecting D-brane models, questions about spacetime instanton effects, induced by Ep-branes, have been largely ignored until quite recently. Since then, there has been a flurry of activity ${ }^{17}$ and this thesis is concerned with some aspects of this $[7,10,9$, , 8 .

\subsection{Overview of this Thesis}

This thesis is concerned with two aspects of intersecting D6-brane models: We treat some of the effects that are induced by E2-instantons and we consider one-loop corrections to the gauge kinetic functions in the low-energy effective field theory.

Recall that Ep-instantons are $\mathrm{D} p$-branes which wrap topologically non-trivial $(p+1)$-cycles of the compactification manifold and are pointlike in four-dimensional spacetime. These Ep-instantons are not physical, in the sense that they are not a part of the background. Rather, they show up in the (fictitious) spacetime string path integral, giving contributions to certain observables weighted by a factor $e^{-C / g_{s}}$. Now, as is well-known (cf. appendix B), such contributions cannot be interpreted as arising from a perturbative expansion in $g_{s}$.

In chapter 3 we present a detailed derivation of the so-called Affleck-Dine-Seiberg (ADS) superpotential [5] as the effect of a single E2-instanon in an intersecting D6-brane realization of supersymmetric QCD (SQCD). This involves a presentation of the formalism necessary for calculating the contribution of E2-instantons to the superpotential and also a construction of SQCD as a (local) intersecting D6-brane model. To give the proper psychological background, we discuss the ADS superpotential in chapter 2, where we have also included some substantial material on other field theory instanton effects.

In chapter 4 we consider one-loop corrections to the gauge kinetic functions in intersecting D6-brane models. These one-loop corrections are related quite intricately to gauge threshold corrections which we compute for intersecting D6-brane models on the $\mathbb{Z}_{2} \times \mathbb{Z}_{2}$ toroidal orbifold. One of the motivations for considering these one-loop corrections is that

\footnotetext{
${ }^{17}$ For a selection of related publications, see the conclusions in chapter 6 .
} 
it is necessary to understand the perturbative expansion before looking at non-perturbative contributions.

Finally, in chapter 5, we take up possible spacetime instanton corrections in intersecting D6-brane models. We examine the holomorphy of E2-instanton contributions to the superpotential and investigate under which circumstances one can expect corrections to the gauge kinetic functions. Interestingly, the above mentioned gauge kinetic functions in $\mathbb{Z}_{2} \times \mathbb{Z}_{2}$ toroidal orbifolds, for example, will not receive any (single) E2-instanton corrections, as there are no three-cycles $\Xi$ with $b_{1}(\Xi)=1$ in such models.

There follow a number of appendices, some elaborating on points touched upon in the main text, such as appendix A, and some collecting formulas necessary for the technical developments in the main text. 
Chapter 2

Some Background: Instantons in Field Theory 


\section{$2.1 \quad$ Introduction}

In the field theory context, instantons (or pseudoparticles) are certain finite action solutions of field theories in Euclidean spacetime, and in the path integral approach to quantum field theory they turn out to be of great dynamical importance. For instance, in a certain lower dimensional analog of QCD, instantons allow for a quantitative understanding of confinement 42]. Unfortunately, despite initial enthusiasm, it turned out that this mechanism cannot work for real QCD. As another example, we mention that instantons lead to baryon non-conservation in QCD [135] (cf. section 2.6) which, although highly suppressed, might still have an impact on our understanding of the early universe.

Broadly speaking, instantons belong to the class of non-perturbative effects 11 Their contribution to the path integral in gauge theories is of the form (see, e.g. [144], chapter 23)

$$
g^{-n} \exp \left(-8|\nu| \pi^{2} / g^{2}\right),
$$

with $g$ the running gauge coupling and $n \in \mathbb{Z}_{>0}, \nu \in \mathbb{Z} \backslash\{0\}$. The precise value of $n$ depends on the particular gauge theory (e.g. $n=12$ for QCD) and $\nu$ is the instanton winding number, a topological number characterizing any given instanton solution. ${ }^{2}$ As explained in appendix B, a function such as (2.1) has no useful expansion in terms of powers of $g$, hence the term 'non-perturbative.'

As the paradigmatic example for instantons, we will first discuss the $S U(2)$ instanton solution due to Belavin, Polyakov, Schwarz, and Tyupkin [24], before considering the contribution of these instantons to the path integral. We then go on to discuss some important effects that instantons have in quantum field theory: The solution of the $U(1)$ problem, the $C P$ problem of QCD, as well as baryon and lepton number violations. Finally, we consider supersymmetry breaking by instantons, that is to say, we present the AffleckDine-Seiberg (ADS) superpotential for supersymmetric QCD.

Thus, the intention of this chapter is to collect the field theory instanton background material upon which the treatment of instantons in string theory constantly relies for intuition. In particular, this chapter serves the purpose of presenting the ADS superpotential in the proper context. For the material in this chapter we have drawn heavily on the excellent treatment given by Rajaraman [123]. We are also indebted to the lectures of Coleman [46], the book by Cheng and $\mathrm{Li}$ [44], and have profited greatly from unpublished lectures by Lüst [107] and Peskin [116]. The original literature is, by any standards, extremely vast and thus we can only cite a comparatively small number of original articles in the sequel. For a more comprehensive collection of gauge theory instanton literature we refer the reader to the reprint volume [128] and references therein.

\footnotetext{
${ }^{1}$ We characterize instantons as non-perturbative effects, as their contributions to the path integral do not show up in perturbation theory. Nevertheless, they are wedded to the semi-classical approximation, i.e. to small gauge coupling.

${ }^{2}$ One can also consider an instanton sector with $\nu=0$ and this gives the perturbative power series expansion in $g$.
} 


\subsection{Instantons in Yang-Mills Theory}

In this section we review the instanton solution, due to Belavin, Polyakov, Schwarz, and Tyupkin (BPST) of Euclidean $S U(2)$ Yang-Mills theory in four dimensions ${ }^{3}$ Since every non-Abelian semi-simple gauge group contains an $S U(2)$ subgroup, this solution will establish the existence of instanton solutions for every Euclidean Yang-Mills theory.

As mentioned in the introduction to this chapter, instantons are finite action solutions of Euclidean field theories. The action here is the Euclidean Yang-Mills action

$$
S\left[A_{\mu}\right]=-\frac{1}{2 g^{2}} \int d^{4} x \operatorname{tr} F_{\mu \nu} F_{\mu \nu},
$$

and the field equations are the Euclidean Yang-Mills equations

$$
D_{\mu} F_{\mu \nu}=\partial_{\mu} F_{\mu \nu}+\left[A_{\mu}, F_{\mu \nu}\right]=0 .
$$

The gauge group is $S U(2)$. Thus, our task is to find solutions $A_{\mu}$ to 2.3 with finite action (2.2.).

For finite action solutions, $F_{\mu \nu}$, which is a function of $A_{\mu}$, clearly has to vanish as $x \rightarrow \infty$. Obviously, this implies the following boundary condition for the gauge potential from which $F_{\mu \nu}$ is derived:

$$
\lim _{x \rightarrow \infty} A_{\mu}(x)=U^{-1}(\hat{x}) \partial_{\mu} U(\hat{x})
$$

where $\hat{x}=x /|x|$. In other words, $A_{\mu}(x)$ must approach a pure gauge at infinity. Pictorially, the siuation looks like in figure 2.1 .

From the argument so far, we see that every field configuration $A_{\mu}$ of finite action defines a map $U(\hat{x})$ of $S^{3}$ into $S U(2)$. It turns out that if we are only interested in finite action solutions up to continuous deformations (without ever violating the requirement of finite action in the deformation process) we can classify them according to the homotopy class ${ }^{4}$ of $U(\hat{x})$. Phrased differently, the homotopy class of the 'gauge transform at infinity' of finite action field configurations provides a topological classification for them.

Since the gauge group $S U(2)$ is semi-simple, there are an infinite number of homotopy classes $\left(\pi_{3}(S U(2))=\mathbb{Z}\right)$, each characterized by an integer called the winding number or Pontryagin index, meaning that the winding number is constant along finite action fields $A_{\mu}$ of the same homotopy class. This winding number $\nu$ is given by

$$
\mathbb{Z} \ni \nu\left(A_{\mu}\right)=-\frac{1}{32 \pi^{2}} \epsilon_{\mu \nu \lambda \rho} \operatorname{tr} \int d^{4} x F_{\lambda \rho} F_{\mu \nu}
$$

\footnotetext{
${ }^{3}$ For remarks on Euclidean field theory, and for our conventions concerning Yang-Mills theory, see appendix C.

${ }^{4}$ We refer to [113] for general topological background.
} 


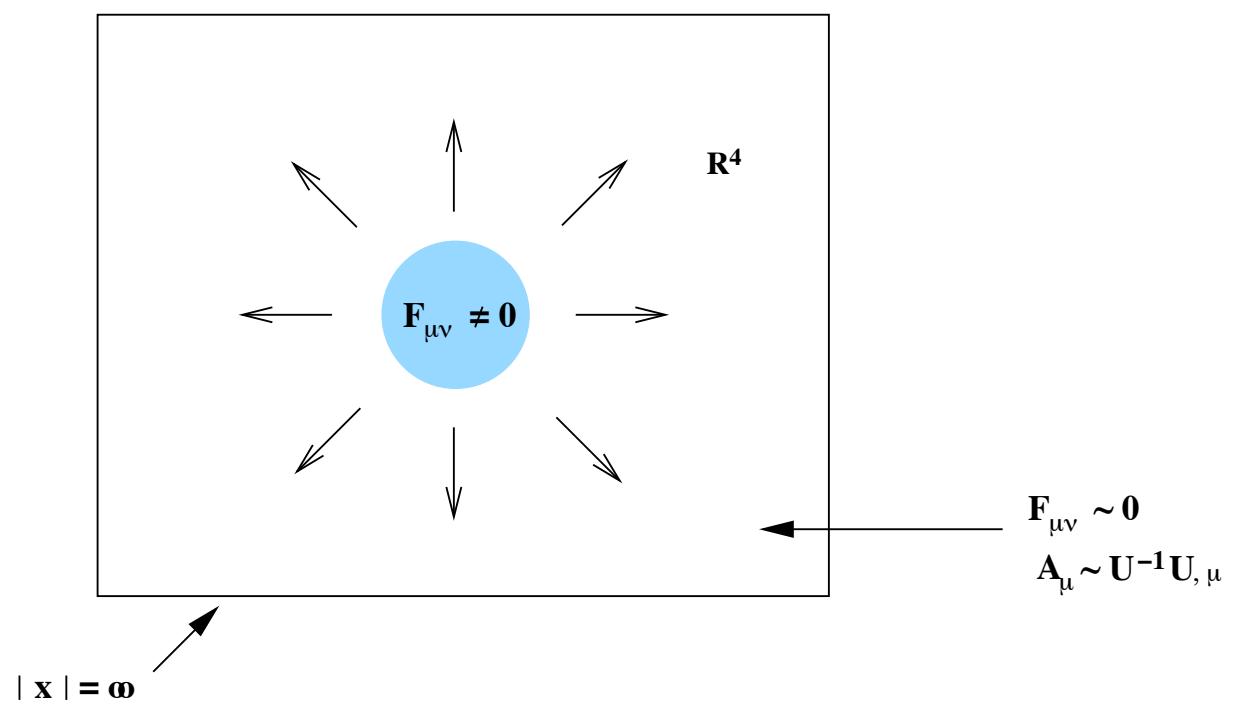

Figure 2.1: Schematic depiction of a Yang-Mills instanton. For very large values of $x$ the field strength asymptotically approaches zero and at infinity the gauge field $A_{\mu}$ is a pure gauge. Adapted from [116].

Note that the winding number is manifestly gauge-invariant. It follows that we can associate a winding number not only with gauge fields $A_{\mu}$ but also with field strengths $F_{\mu \nu}$.

What we have said up until now applies to all finite action field configurations, not only to solutions of the Euclidean Yang-Mills equation (2.3). Now we want to look for finite action field configurations actually satisfying the Euclidean Yang-Mills equations (2.3).

Since we cannot hope to obtain a complete overview of all such solutions, we settle for getting any solutions at all. To this end, consider the relation

$$
-\operatorname{tr} \int d^{4} x\left(F_{\mu \nu} \pm \widetilde{F}_{\mu \nu}\right)^{2} \geq 0
$$

where $\widetilde{F}_{\mu \nu}=\frac{1}{2} \epsilon_{\mu \nu \lambda \rho} F_{\lambda \rho}$. From (2.6) and 2.5) it follows after some manipulation that

$$
S\left[A_{\mu}\right] \geq\left(8 \pi^{2} / g^{2}\right)\left|\nu\left(A_{\mu}\right)\right| .
$$

We now look for field configurations saturating the bound in (2.7). It can be shown that for any given value of $\nu$ the lower bound in (2.7) is attained precisely if the field configuration satisfies either the self-duality $(+)$ or anti self-duality $(-)$ relation

$$
\widetilde{F}_{\mu \nu}= \pm F_{\mu \nu}
$$

where + is to be chosen for positive $\nu$ and - for negative $\nu$. Moreover, any $F_{\mu \nu}$ satisfying one or the other relation in (2.8) automatically satisfies the field equations (2.3). Note, however, that the converse is not true: It is not true that every finite action solution of 
the Euclidean Yang-Mills equations (2.3) has to be (anti) self-dual. In fact, explicit nonselfdual finite-action solutions of the Euclidean Yang-Mills equations are known and we refer to section 2.1 of the review [141] for some discussion. However, it is also known that any such non self-dual solutions are only saddle point of the Yang-Mills action.

In practice, the existence of non-selfdual finite action solutions of the Euclidean Yang-Mills equations is of no great practical import since, as will be discussed in section 2.3, one is interested in instantons in the context of the Euclidean path integral because they are stationary points of the action and in any given topological sector of winding number $\nu$ the (anti) self-dual instantons have the absolutely lowest action..$^{5}$ One often even goes so far as calling only the (anti) self-dual solutions of the Euclidean Yang-Mills equations by the name of (anti-) instanton, and we shall also do so. Figure 2.2 illustrates the question about the existence of finite action solutions of the Euclidean Yang-Mills equations which are not (anti) self-dual.

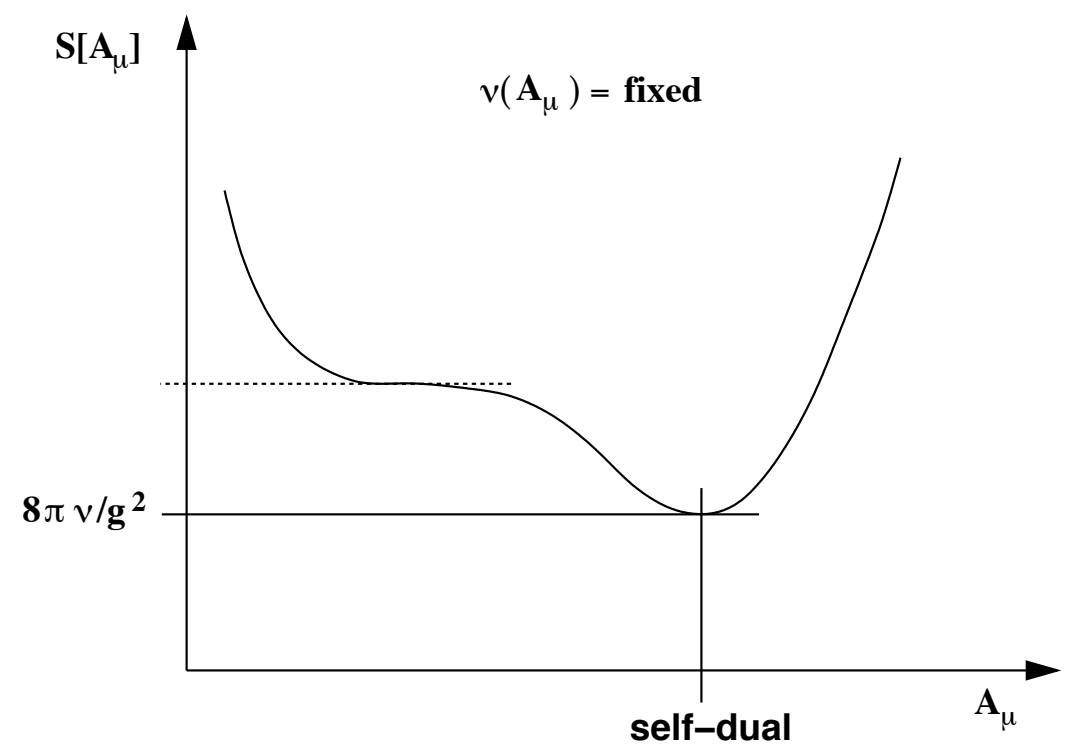

Figure 2.2: In a given topological sector (fixed winding number $\nu$ ) of finite action field configurations, among all solutions of the Yang-Mills equations, those which are (anti) self-dual have the absolutely lowest action. There can also be non-self-dual solutions with finite action, here indicated by the dashed line, but they correspond to saddle-points of the action.

For winding number $\nu=1$ a one-parameter family of solutions to the self-duality equation 2.8) was given by BPST themselves [24]. It can be represented for the self-dual (instanton) case by

$$
A_{\mu}=\frac{r^{2}}{r^{2}+\rho^{2}} U^{-1}(x) \partial_{\mu} U(x)
$$

\footnotetext{
${ }^{5}$ In particular, since any non-self-dual solutions correspond only to saddle points of the action, they cannot be taken as mid-points for calculating quantum-fluctuations around them.
} 
where $U(x)=\frac{x_{4}+i \vec{x} \cdot \vec{\sigma}}{r}, r^{2}=x_{4}^{2}+\vec{x}^{2}$, and $\vec{\sigma}$ denotes the usual vector of Pauli matrices. Note that the parameter $\rho$ is dimensionful. Thus, each such instanton carries with it a scale, implying that it is a long-range field. There is also a corresponding anti self-dual (i.e. anti-instanton) family with $\nu=-1$ which can be obtained by replacing $U(x)$ by $U^{-1}(x)$ in 2.9. It is worth remarking explicitly that every field configuration in 2.9 has action $S\left[\overline{A_{\mu}}\right]=8 \pi^{2} / g^{2}$, which confirms their non-perturbative nature.

For $|\nu|>1$, the ADHM construction [18] allows to generate solutions which we shall not write down as they are unfortunately rather complicated. In practice, often only the existence of such solutions is important. For existence, one could argue that one only needs to superimpose $|\nu|=1$ solutions with centers far enough apart. Then, since the winding number is additive, we would get general $\nu$ solutions. The trouble with this argument is that it is not clear whether these superimposed field configurations are stable, that is, whether the non-linearities in the Yang-Mills equations can consistently be ignored for such configurations. Therefore, it is necessary to know that indeed such solutions exist, each, in accordance with (2.7), having action $S\left[A_{\mu}\right]=8 \pi^{2}|\nu| / g^{2}$

At the beginning of this section, we remarked that the $S U(2)$ instantons so-far presented show that there are instantons in any Euclidean Yang-Mills theory, given that its gauge group $G$ is semi-simple, i.e. contains an $S U(2)$ subgroup. Indeed, suppose that we have identified some $S U(2)$ subgroup of $G$, then the, say, $\nu=1$ instanton solution is easily extended to this case by taking $A_{\mu}$ to be equal to 2.9 for the $S U(2)$ subgroup and all other matrix elements equal to zero.

Until now we have somewhat abstractly discussed the instanton solution of BPST without giving any physical interpretation. However, there is an interpretation of instantons in terms of quantum tunneling which we consider now. First of all, the multi-instanton solution can be interpreted as a path in Euclidean space connecting two vacua of topological winding number differing by precisely the winding number of the multi-instanton (cf. [44] for the following). We can explain this in formulas for the single instanton:

In temporal $\left(A_{0}=0\right)$ gauge ${ }^{6}$ one has for the single instanton solution

$$
A_{i}(x)=U^{-1}(x) \partial_{i} U(x),
$$

with

$$
U\left(x^{4}=-\infty\right)=\exp \left(i \pi \frac{\vec{x} \cdot \vec{\sigma}}{\sqrt{\vec{x}^{2}+\rho^{2}}} \nu\right)
$$

and

$$
U\left(x^{4}=+\infty\right)=\exp \left(i \pi \frac{\vec{x} \cdot \vec{\sigma}}{\sqrt{\vec{x}^{2}+\rho^{2}}}(\nu+1)\right) .
$$

Here, $\nu$ is an integration constant coming from the transformation to temporal gauge.

\footnotetext{
${ }^{6}$ One also requires the spatial part of the gauge field to vanish in the infinite past and future.
} 
One interprets these formulas as meaning that the instanton solution interpolates between different topological sectors of the gauge theory, each labeled by a particular winding number $\nu$. In this sense we write:

$$
\begin{aligned}
& \lim _{x^{4} \rightarrow-\infty} A(x) \leftrightarrow \text { sector with winding number } \nu \\
& \lim _{x^{4} \rightarrow+\infty} A(x) \leftrightarrow \text { sector with winding number } \nu+1
\end{aligned}
$$

Transition amplitudes between topological sectors can be calculated in the semiclassical approximation and are roughly given by minus the exponential of the action of the interpolating instanton (we will not demonstrate this calculation here). Since there are many topologically inequivalent vacua in the gauge theory (i.e. field configuration upon which a perturbation theory of small quantum fluctuations can be built), none of them can be considered as 'the' vacuum. The situation is reminiscient of the quantum mechanics of a double well. There, wave functions localized in any of the two humps can be considered as 'vacua'; since there is a non-zero transition amplitude for tunneling between them, none is fundamental. Rather, a linear superposition gives a good ground state wave function.

Now, in the gauge theory context the situation is analogous and one has an infinity of gauge theory vacua $|\theta\rangle$, labeled by the arbitrary real number $\theta$, each a linear superposition of topologically inequivalent vacua $|\nu\rangle$, labeled by their winding number $\nu$ :

$$
|\theta\rangle=\sum_{\nu=-\infty}^{+\infty} e^{i \theta \nu}|\nu\rangle .
$$

Note that this wave function is analogous to the wave function of a particle in a periodic potential (Bloch waves). We mention also that there can be transitions between between different $\theta$-vacua.

All these remarks about the physical relevance of the instanton solutions of pure Yang-Mills theory suggest that its vaccum struture is extremely rich.

\subsection{Instantons in the Euclidean Path Integral}

In the previous section we reported on the existence of finite action solutions (i.e. stationary points of the action) for Euclidean Yang-Mills theories. In this section we want to comment on their relevance in the path integral approach to quantum field theory.

The basic object of study in the path integral approach to quantum field theory is the partition function (we let $\phi$ collectively stand for the fields in our theory)

$$
Z=\int[d \phi] e^{i S_{\text {Minkowski }}[\phi]}
$$


that is, a weighted 'sum' of field configurations, where the weight-factor is the exponential of $i$ times the action for the given field configuration.

Now, for some applications, such as the evaluation of the ground state energy, it is useful (or necessary) to go to the Euclidean version of the path integral

$$
Z_{\mathrm{Min}}=\int[d \phi] e^{i S_{\text {Minkowski }}[\phi]} \rightarrow Z_{\mathrm{Euc}}=\int[d \phi] e^{-S_{\text {Euclid }[\phi]}}
$$

The Euclidean path integral then determines the vacuum energy density via the equation

$$
Z_{\text {Euc }}=\exp [-(\text { volume of } 4 \text {-space }) \times(\text { vacuum energy density })] .
$$

(More generally, by introducing a source term, we could obtain the generating functional for Green's functions.)

For Yang-Mills theory, the Euclidean path integral is

$$
Z=\int\left[d A_{\mu}\right] e^{-\frac{1}{2 g^{2}} \int d^{4} x \operatorname{tr} F_{\mu \nu} F_{\mu \nu}},
$$

which, due to the gauge nature of $A_{\mu}$, would still have to be subjected to some gauge fixing procedure; this is not important for our current discussion. We shall denote the argument of the exponential by $S\left[A_{\mu}\right]$ in the following.

If we assume that the coupling constant $g \ll 1$, we can attempt a saddle-point approximation for 2.19). In this approximation, we expand the path integral in a sum of terms of path integrals around the stationary points $A_{\mu}$ of the action with $S\left[A_{\mu}\right]<\infty$, as these are weighted highest by the exponential. Thus, in this approximation

$$
Z=\sum_{\substack{\delta S\left[A_{\mu}^{k}\right] / \delta A_{\mu}^{k}=0 \\ S\left[A_{\mu}\right]<\infty}} \int\left[d A_{\mu}^{k}\right] e^{-S\left[A_{\mu}^{k}\right]} .
$$

At first glance, one might suspect that this sum reduces to precisely one term, namely the one for the trivial stationary point $A_{\mu} \equiv 0$. This term leads to the usual perturbative expansion of the path integral in powers of $g$ (and corresponds to winding number $\nu=0$ ). However, in section 2.2 we learned that, in fact, the (Euclidean) Yang-Mills action has non-trivial stationary finite action points, namely the instantons. Thus the index $k$ in equation 2.20 can be replaced by the winding number $\nu$, and since in any given topological sector the instantons have the absolutely lowest action, we can take them to be the basis of the saddle-point approximation.

In summary, we then have

$$
Z=\int\left[d A_{\mu}\right]_{\nu=0} e^{-S\left[A_{\mu}\right]}+\int\left[d A_{\mu}\right]_{|\nu|=1} e^{-S\left[A_{\mu}\right]}+\cdots
$$


For each topological sector, the minimal action is attained for the instanton solution with the value

$$
-\left.\frac{1}{2 g^{2}} \int d^{4} x \operatorname{tr} F_{\mu \nu} F_{\mu \nu}\right|_{\min }=\frac{8 \pi^{2}}{g^{2}}|\nu|,
$$

so, as remarked already several times, the non-trivial topological sectors give a nonperturbative contribution to the path integral, that is, a contribution which would not show up in perturbation theory, even though the coupling constant is assumed to be very small. We can already see from (2.21) that instantons have the potential to give nonvanishing contributions to observables when the perturbative expansion fails to do so. This is a particularly interesting possibility to break symmetries and we will discuss some such effects later on.

The vacuum energy due to instantons. In general, it is quite difficult to work out the integrals in (2.21), as they lead, amongst other things, to infinite determinants. Since we have taken the instantons as the 'midpoints', so to speak, of the path integrals in (2.21), evaluating these integrals amounts to considering quantum fluctuations around these 'midpoints'. To do these integrals, it is necessary to know in detail the free parameters in the instanton solutions, which we have not described (for winding number $\nu=1$ we only mentioned the scale $\rho$ but one can also subject the given solution to translations and rotations). The usual term for these parameters is 'collective coordinates'. In order to get a feel of what is involved, we want to sketch the result one gets. We are following [116] here. For the $\nu=1$ sector in (2.21) it is

$$
\int\left[d A_{\mu}\right]_{\nu=1} e^{-S\left[A_{\mu}\right]}=\int d^{4} x_{0} \frac{d \rho}{\rho^{5}} \int d^{3} \Omega e^{-8 \pi^{2} / g^{2}}(\operatorname{det}[])^{-1 / 2},
$$

where $(\operatorname{det}[])^{-1 / 2}$ is the result of integrations over the quantum fluctuations about the instanton solution, ultimately related to a change of variables from fields to collective coordinates. One effect of this is the replacement

$$
\frac{8 \pi^{2}}{g^{2}} \rightarrow \frac{8 \pi^{2}}{g^{2}(\rho)}
$$

where $g(\rho)$ is the running coupling of QCD evaluated at the scale $\rho$. This is one expression of the fact that the instanton is a long range field, which we indicated in section 2.2. The factor $1 / \rho^{5}$ is required by dimensional analysis. From the $\nu=-1$ sector there will be a similiar contribution.

For $\nu= \pm 2$ one can write

$$
\int\left[d A_{\mu}\right]_{|\nu|=2} e^{-S\left[A_{\mu}\right]} \simeq\left[\int d^{4} x_{0} \frac{d \rho}{\rho^{5}} \int d^{3} \Omega e^{-8 \pi^{2} / g^{2}} C\right]^{2}
$$

for instantons with centers far apart plus a small correction for when they are near. Also, in the $\nu=0$ sector there is a non-perturbative correction from widely separated instantonanti-instanton pairs. 
If one only keeps the terms for widely seperated instantons, one gets

$$
\begin{aligned}
Z & =\sum_{\nu_{+}, \nu_{+}} \frac{1}{\nu_{+} ! \nu_{-} !}\left[\int d^{4} x_{0} \frac{d \rho}{\rho^{5}} \int d^{3} \Omega e^{-8 \pi^{2} / g^{2}} C\right]^{\nu_{+}+\nu_{-}} \\
& =\exp \left[-(\text { vol. of } 4 \text {-space })(-2)\left(\int \frac{d \rho}{\rho^{5}} \int d^{3} \Omega e^{-8 \pi^{2} / g^{2}} C\right)\right],
\end{aligned}
$$

from which we read off the vacuum energy density in the presence of instantons as

$$
\text { vacuum energy density }=(-2)\left(\int \frac{d \rho}{\rho^{5}} \int d^{3} \Omega e^{-8 \pi^{2} / g^{2}}\right) .
$$

Thus, instantons give a negative, non-perturbative contribution to the vacuum energy density. The integral over $\rho$ is, of course, divergent but for small- $\rho$ instantons the contribution is well-defined.

\subsection{Instanton Resolution of the $U(1)$ Problem}

In section 2.2 we remarked that quite often one can already infer important facts from the existence of instanton solutions to Yang-Mills theories, i.e. without knowing the explicit form of these solutions. One such instance is the so-called $U(1)$ problem [136, 50, 45, 145].

Let us first briefly sketch the problem. Let us consider the sector of QCD dealing only with the up and down quarks, and approximate their masses to zero

$$
m_{u}, m_{d} \simeq 0 .
$$

The part of the QCD Lagrangian $\mathcal{L}_{\mathrm{QCD}}$ dealing with this sector then has the symmetry $S U(2)_{L} \times S U(2)_{R} \times U(1)_{V} \times U(1)_{A}$, which is larger than just the chiral symmetry $S U(2)_{L} \times$ $S U(2)_{R}$.

The $U(1)_{V}$ symmetry $\left(\right.$ quark $_{i} \rightarrow e^{i \alpha}$ quark $_{i}$ ) gives a conserved current

$$
J_{\mu}^{B}=\bar{u} \gamma_{\mu} u+\bar{d} \gamma_{\mu} d,
$$

whose conservation corresponds to baryon number conservation.

The $U(1)_{A}\left(\right.$ quark $_{i} \rightarrow e^{i \beta \gamma_{5}}$ quark $\left._{i}\right)$ current is

$$
J_{\mu}^{5}=\bar{u} \gamma_{m} u \gamma_{5} u+\bar{d} \gamma_{m} u \gamma_{5} d
$$

but it does not correspond to any observed symmetry in hadron spectra.

For this reason, one expects the $U(1)_{A}$ symmetry to be spontaneously broken, giving rise to a massless pseudoscalar (Goldstone boson) in addition to the pion triplet $\pi^{+}, \pi^{-}, \pi^{0}$ 
(arising from the breaking of the $\left.S U(2)_{L} \times S U(2)_{R}\right)$. In addition, we expect this particle to be of mass comparable to $\pi^{ \pm}, \pi^{0}$, as they all have the same quark composition.

In nature, there does not exist such a particle! This is referred to as the $U(1)$ problem. Sometimes one also speaks of the $\eta$-mass problem, as the $\eta$ particle has the right quantum numbers except for its mass. However, as pointed out in [46], this is really just a misnomer for the problem, since the $\eta$ has nothing to do with its solution.

The way out of the conundrum posed by the $U(1)$ problem is that the $U(1)_{A}$ symmetry can be broken by instantons without generating any Goldstone bosons.

The axial current (2.31) suffers from the Adler-Bell-Jackiw anomaly

$$
\partial_{\mu} J_{\mu}^{5}=\frac{N_{f} g^{2}}{16 \pi^{2}} F_{\mu \nu}^{a} \widetilde{F}_{\mu \nu}^{a}
$$

In a topologically trivial $(\nu=0)$ gauge field sector the right hand side of 2.32 gives zero, but for $\nu \neq 0$ it gives something non-vanishing, implying axial-charge violation. This in turn means that the $U(1)_{A}$ symmetry is not a good quantum-symmetry and thus need not be broken at all by the Higgs mechanism. Therefore, no pseudoscalar particle is generated and the $U(1)_{A}$ problem evaporates.

\subsection{The CP Problem of QCD}

It is a principle of quantum field theory that all possible terms respecting the symmetries of a system should be added to the Lagrangian density. In addition to this one also often requires renormalizability of the Lagrangian.

If one applies this principle to QCD, taking gauge invariance under $S U(3)$ transformations as the symmetry principle, the following mass dimension four term should appear in the Lagrangian density $\mathcal{L}$, where for later convenience we work in Euclidean space:

$$
\Delta \mathcal{L}_{\theta}=\frac{\theta}{32 \pi^{2}} \int d^{4} x F_{\mu \nu} \widetilde{F}_{\mu \nu}
$$

Here $\theta$ is some, at first arbitrary, real parameter. For non-vanishing $\theta$ this term turns out to be $C P$ violating and if we took $C P$ conservation as a symmetry principle of the strong interactions we would not be allowed to add it in defining the theory. Still, the term could be generated by weak interactions and we shall comment on this later.

In the topological sector of QCD with winding number $\nu=0$, the term (2.33) vanishes identically but for $\nu \neq 0$ it gives a non-vanishing contribution to the Euclidean path integral. This is so because (cf. (2.5))

$$
\frac{\theta}{32 \pi^{2}} \int d^{4} x F_{\mu \nu} \widetilde{F}_{\mu \nu} \sim \text { winding number } \nu \text {. }
$$


Before remarking on the theoretical problems posed by the term (2.33) let us first present a way of thinking about it which differs from adding all terms having the required symmetries. In section 2.2 we introduced the idea of $\theta$-vacua of gauge-theories. This idea holds good in particular in QCD, i.e. $S U(3)$ gauge theory. The interpretation of $(2.33)$ comes from calculating the following transition amplitude for $\tau \rightarrow \infty$ [44]

$$
\begin{aligned}
\left\langle\theta\left|e^{-H \tau}\right| \theta\right\rangle & =\sum_{\nu, \bar{\nu}} e^{-i \nu \theta}\left\langle\nu+\bar{\nu}\left|e^{-H \tau}\right| \nu\right\rangle \\
& =2 \pi \delta(0) \sum_{\nu} e^{-i \nu \tau} \int\left[d A_{\mu}\right]_{\bar{\nu}} e^{-S_{\text {Euc }}} \\
& =2 \pi \delta(0) \int\left[d A_{\mu}\right]_{\text {all } \bar{\nu}} \exp \left(-S_{\text {Euc }}+\frac{i \theta}{16 \pi^{2}} \int d^{4} x F_{\mu \nu} \widetilde{F}_{\mu \nu}\right),
\end{aligned}
$$

where $H$ is the Euclidean Hamiltonian.

We interpret this as meaning that if we accept the physical existence of an infinity of $\theta$ vacua, the quantum theory corresponding to each $\theta$-vacuum is obtained by adding to the usual QCD Lagrangian a $\theta$-term 2.33 .

Now, from experimental upper limits on the neutron's dipole moment one can deduce an upper bound on the $\theta$ parameter of QCD [51]:

$$
|\theta|<10^{-9}
$$

meaning that the interactions in QCD can only be very slightly $C P$ violating. If one takes the usual definition of naturalnes: ${ }^{7}$ at face value, the smallness of $\theta$ is not at all natural. This is referred to as the strong $C P$ problem of QCD.

Over the years, several mechanisms have been proposed to explain the smallness of $\theta$. One line of reasoning, beginning with Peccei and Quinn [115], in effect stipulates that $\theta$ should be a dynamical field with some potential which allows $\theta$ to relax to zero. This in turn predicts a number of particles called axion 8 for which one can then experimentally search (for a recent overview of axionic physics, see [103]). The original Peccei-Quinn axion is by now experimentally ruled out, but the axion idea is still very compelling.

Another way out of the puzzle posed by $\theta$ is, as already remarked, to postulate as a symmetry principle for QCD the conservation of $C P$. Since $(2.33)$ does not respect $C P$, it would not be allowed to add this term. Of course, proceeding in this way would run afoul of the interpretation of the $\theta$-term in terms of $\theta$-vacua, but there is another problem: The weak interaction is manifestly $C P$ violating and higher order weak interactions generate $\gamma^{5}$-dependent quark masses and their elimination in effect again introduces a $\theta$-term. In

\footnotetext{
${ }^{7}$ The smallness of some dimensionless parameter is considered natural if setting it to zero enhances the system's symmetries ('t Hooft).

${ }^{8}$ The term axion, apparently the brand name for a detergent, was chosen by Frank Wilczek because it could potentially 'clean up' the problem of the smallness of $\theta$.
} 
order for $\theta$ to be small, the weak $C P$-violations must be soft and it appears as if this is extremely difficult to realize. One refers to this as the weak $C P$ problem. In effect, one has to explain why the sum of the $\theta$ 's of the strong and weak sectors are small (cf., say, [141]).

Thus, it appears as if the existence of instantons in Yang-Mills theory poses yet another puzzle for high-energy theory. The fact that the $C P$ problem has resisted a complete and experimentally vindicated solution for such a long time might point to a real deficiency in the understanding of particle physics or to the understanding of what is natural. On the other hand, it might as well turn out that some variant of the Peccei-Quinn idea is correct and will be vindicated by the discovery of axionic particles. This would of course be the most wonderful resolution of the puzzle since then the theoretical solution of a theoretical problem would have had made a prediction for observable physics.

\subsection{Baryon and Lepton Number Violations}

We remarked in section 2.3 that instantons have the potential to break symmetries even when these symmetries are good at the perturbative level. 't Hooft has found an important effect that instantons have in gauge theries when fermions are are present (such as QCD) [134]. Consider a theory with $N_{f}$ flavors of massless quarks. The theory has a chiral symmetry

$$
S U\left(N_{f}\right)_{L} \times S U\left(N_{f}\right)_{R} \times U(1) .
$$

The axial current $J_{\mu}^{5}$ suffers from the Adler-Bell-Jackiw anomaly

$$
\partial_{\mu} J_{\mu}^{5}=\frac{N_{f} g^{2}}{16 \pi^{2}} F_{\mu \nu}^{a} \widetilde{F}_{\mu \nu}^{a}
$$

Comparing with the equation for the winding number $\nu(2.5)$ gives

$$
\int d^{4} x \partial_{\mu} J_{\mu}^{5}=2 N_{f} \nu
$$

so that in a background field of winding number $\nu=1$ the axial charge is violated by the amount

$$
\Delta Q^{5}=2 N_{f}
$$

This violation results in decays such as

$$
p \rightarrow e^{+}+\bar{\nu}_{\mu}, \text { or } \mu^{+}+\bar{\nu}_{e},
$$

which violate baryon and lepton number conservation.

These decays, however, are highly suppressed due to the small weight $\left(e^{-[\ldots]}\right)$ the instanton carries in the path integral. Because the instanton induced number violations at first really 
only show up at the level of the basic theory of quarks and leptons some modelling for, say, the proton has to be applied to infer the import of these violations on its lifetime. Thus, one can expect a variance of some orders of magnitude in the predictions for experiment. In effect, the lifetime of the deuteron (the nucleus of ${ }^{3} \mathrm{He}$ ) comes out roughly as $10^{218}$ years (cf. [124]), a lifetime so great that it appears unlikely that there will be an experimental verification of instantons in this arena.

\subsection{ADHM Construction}

We give here some cursory remarks on the so-called ADHM construction [18]. In fact, in keeping with the goal of this chapter to provide psychological background we want to give some anecdotal meaning to the jargon to be used later, notably in chapter 3 .

The ADHM construction is a method for constructing self-dual solutions to the Euclidean Yang-Mills equations, i.e. instantons. In fact, it has been shown that it generates all solutions with a given winding number.

Any instanton solution contains a number of parameters, the so-called collective coordinates, over which one has to integrate in the path integral. Indeed, we already commented on the parameters of the BPST solution, and we integrated over them in section 2.3. Of course, one really has to integrate over fields in the path integral but this is reduced to an integration over collective coordinates by a (difficult) change of variables.

Now, the instanton solutions resulting from the ADHM construction are only implicitly defined by the so-called ADHM constraints which really are constraints on the collective coordinates. Thus, in using instanton solutions coming out of the ADHM construction in path integrals, one must take into account these constraints in the integration over collective coordinates.

The ADHM construction has been extended to super Yang-Mills theories [126, 142]. For these theories, the collective coordinates of the instantons are bosonic and fermionic (Grassmannian), and one consequently has bosonic as well as fermionic constraints.

In chapter 3 we will encounter ADHM constraints on the E2-instanton zero modes. The origin of these constraints, however, does not lie with an explicit construction. Rather, they arise from D-term and F-term constraints (for the bosonic zero modes). This is, in fact, related to the physical understanding of the ADHM construction provided by D-branes.

Integrating over collective coordinates amounts to taking into acount quantum fluctuations of the instanton. Clearly, for E2-instantons the integration over zero modes also corresponds to quantum fluctuations around the classical configuration. 


\subsection{The ADS Superpotential}

At least at low energies nature is not supersymmetric - there is no observed mass degeneracy between bosons and fermions. Therefore, breaking of supersymmetry in supersymmetric gauge field theories is of great importance if they are to play any role in a theory of nature.

We have already seen in a number of instances (sections 2.4, 2.5, 2.6) that instantons have the potential to break symmetries in quantum field theory. The dynamical breaking of supersymmetry by non-perturbative effects is particularly attractive as it naturally leads to hierarchies in supersymmetric gauge theories [148]. It therefore seems natural to ask whether supersymmetry can be broken by instantons, i.e. by a particular non-perturbative effect. The question of non-perturbative supersymmetry breaking was taken up by Affleck, Dine, and Seiberg (ADS) [5, 4] and they found that in particular instances this indeed is realized 9

In this section we shall explain the findings of ADS concerning the breaking of supersymmetry by instantons in supersymmetric QCD (SQCD) ${ }^{10}$ We begin by describing SQCD and its symmetries in some detail.

SQCD is a supersymmetric 'generalization' of ordinary QCD. As such it is a theory of $N_{f}$ flavors of chiral 'superquarks' $\Phi_{f}$ and $N_{f}$ flavors of chiral 'anti-superquarks' $\widetilde{\Phi}_{f}$ coupled to $S U\left(N_{c}\right)$ super Yang-Mills theory. The $\Phi_{f}$ are taken to transform in the fundamental representation $\mathbf{N}_{\mathbf{c}}$ of $S U\left(N_{c}\right)$ and the $\widetilde{\Phi}_{f}$ are taken to transform in the anti-fundamental representation $\overline{\mathbf{N}}_{\mathbf{c}}$ of $S U\left(N_{c}\right)$ :

$$
\begin{array}{ll}
N_{f} \text { chiral superfields } \Phi_{f} \in & \mathbf{N}_{\mathbf{c}} \\
N_{f} \text { chiral superfields } \widetilde{\Phi}_{f} \in & \overline{\mathbf{N}}_{\mathbf{c}}
\end{array}
$$

Note that for instance $\Phi_{f}$ implicitly carries also a color index $c$, such that $\Phi_{f}=\Phi_{f c}$ and thus effectively it is a matrix valued field. For $\widetilde{\Phi}_{f}$ we take the index structure to be $\widetilde{\Phi}_{f}=\widetilde{\Phi}_{c f}$, just the opposite as for $\Phi_{f}$.

We denote the scalar components (squarks) of the chiral fields by $\phi_{f}, \widetilde{\phi}_{f}$ and their fermionic components (quarks) by $\psi_{f}, \widetilde{\psi}_{f}$ :

$$
\begin{aligned}
& \Phi_{f}=\left(\phi_{f}, \psi_{f}\right) \\
& \widetilde{\Phi}_{f}=\left(\widetilde{\phi}_{f}, \widetilde{\psi}_{f}\right) .
\end{aligned}
$$

\footnotetext{
${ }^{9}$ Important input also was due to Davis, Dine, and Seiberg 59 .

${ }^{10}$ In some instances supersymmetry is broken by non-perturbative effects different from instantons, e.g. by gaugino-condenstation. Since this is outside of our main concern in this thesis we will have little to say about this
} 


\begin{tabular}{c|c|ccc} 
& $S U\left(N_{c}\right)$ & $S U\left(N_{f}\right)$ & $S U\left(N_{f}\right)$ & $U(1)_{V}$ \\
\hline$\phi_{f}, \psi_{f}$ & $\square$ & $\square$ & 1 & 1 \\
$\widetilde{\phi}_{f}, \widetilde{\psi}_{f}$ & $\square$ & 1 & $\square$ & -1
\end{tabular}

Table 2.1: Good quantum symmetries of SQCD with $N_{f}$ flavors of chiral superquarks $\Phi_{f}=\left(\phi_{f}, \psi_{f}\right)$ and $N_{f}$ flavors of chiral anti-superquarks $\widetilde{\Phi}_{f}=\left(\widetilde{\phi}_{f}, \widetilde{\psi}_{f}\right)$. The symbol stands for the fundamental representation of $S U\left(N_{c}\right)$, while $\square$ denotes the anti-fundamental representation. For the meaning of $U(1)_{V}$, see 2.52 .

The Lagrangian of the theory is

$$
\mathcal{L}=\int d^{4} \theta \sum_{f=1}^{N_{f}}\left(\bar{\Phi}_{f} e^{V} \Phi_{f}+\widetilde{\Phi}_{f} e^{V} \overline{\widetilde{\Phi}}_{f}\right)+\frac{\tau}{32 \pi i} \int d^{2} \theta\left(\operatorname{tr} \mathcal{W}_{\alpha}^{2}+\text { h.c. }\right)
$$

Here $V$ is the $\mathcal{N}=1$ vector superfield transforming in the fundamental representation of $S U\left(N_{c}\right)$ and $\mathcal{W}_{\alpha}$ is the super field strength, while $\tau$ is the usual complex coupling. We denote hermitian conjugation by a bar $\left(^{-}\right)$, while, to be explicit, the tilde $\left(^{\sim}\right)$ is supposed to indicate that a field transforms in the antifundamental representation of $S U\left(N_{c}\right)$. Note that the first part of the Lagrangian is responsible for the dynamics of the (anti-) superquarks and their coupling to $S U\left(N_{c}\right)$ super Yang-Mills, whereas the second part is just 'supergluodynamics.' We could also have added a superpotential term to the Lagrangian which, however, we choose to be equal to zero. It can be shown that if the superpotential is zero at the classical level it is zero to all orders in perturbation theory. Now, since the theory is manifestly supersymmetric at the classical level and no supersymmetry breaking superpotential will be induced in perturbation theory, the only way such a term can be generated is via non-perturbative effects.

Good quantum symmetries. The theory just written down has a number of symmetries. First there are symmetries, summarized in table 2.8, for the component squark and quark fields as well as their 'anti-' counterparts, which are also good symmetries of the quantum theory. The gauge $S U\left(N_{c}\right)$ symmetry acts in the usual way on the chiral fields:

$$
\Phi_{f c} \rightarrow U_{c c^{\prime}} \Phi_{f c^{\prime}}, \quad \widetilde{\Phi}_{c f} \rightarrow U_{c c^{\prime}}^{*} \widetilde{\Phi}_{c^{\prime} f}
$$

with $U \in S U\left(N_{c}\right)$. In addition, there are also global $S U\left(N_{f}\right)$ flavor rotations

$$
\Phi_{f} \rightarrow U_{f f^{\prime}} \Phi_{f^{\prime}}, \quad \widetilde{\Phi}_{f} \rightarrow \widetilde{\Phi}_{f}
$$

and

$$
\Phi_{f} \rightarrow \Phi_{f}, \quad \widetilde{\Phi}_{f} \rightarrow U_{f f^{\prime}} \widetilde{\Phi}_{f^{\prime}},
$$

with $U \in S U\left(N_{f}\right)$. Finally, we have the vector-like $U(1)_{V}$ symmetry

$$
\Phi_{f} \rightarrow e^{i \alpha} \Phi_{f}, \quad \widetilde{\Phi}_{f} \rightarrow e^{-i \alpha} \widetilde{\Phi}_{f},
$$

with $\alpha$ some real parameter. Let us emphasize again that it can be shown that all these are valid symmetries of SQCD not only at the classical but also at the quantum level. 
Anomalous symmetries. There are also a number of symmetries of SQCD which are perturbatively violated. First, we have the so-called $U(1)_{R}$ symmetry

$$
\psi \rightarrow e^{-i \alpha} \psi, \quad \widetilde{\psi} \rightarrow e^{-i \alpha} \widetilde{\psi}, \quad \lambda \rightarrow e^{i \alpha} \lambda
$$

with $\alpha$ some real parameter and $\lambda \subset \mathcal{W}_{\alpha}$ the gluino field.

Second, we have the axial-like $U(1)_{A}$ symmetry

$$
\Phi_{f} \rightarrow e^{i \alpha} \Phi_{f}, \quad \widetilde{\Phi}_{f} \rightarrow e^{i \beta} \widetilde{\Phi}_{f}
$$

with $\alpha, \beta$ two independent real paramters. Note that here, unlike for the $U(1)_{V}$, the superquarks and anti-superquarks transform independently.

As already emphasized, these symmetries are anomalous in the quantum theory. It turns out, however, that they can be combined to give an anomaly free symmetry, called the 'non-anomalous $U(1)_{R}$ symmetry:'

$$
\begin{aligned}
& \Phi_{f}(x, \theta) \rightarrow e^{i \alpha \frac{N_{f}-N_{c}}{N_{f}}} \Phi_{f}\left(x, \theta e^{-i \alpha}\right), \\
& \widetilde{\Phi}_{f}(x, \theta) \rightarrow e^{i \alpha \frac{N_{f}-N_{c}}{N_{f}}} \widetilde{\Phi}_{f}\left(x, \theta e^{-i \alpha}\right) .
\end{aligned}
$$

Note that this involves the number of flavors $N_{f}$ and number of colors $N_{c}$ as parameters.

Non-perturbative Superpotential. Now that we have collected a number of good quantum symmetries of SQCD we can consider [59] what kind of superpotential can be generated non-perturbatively (recall that with our definition of SQCD 2.48) the superpotential vanishes to all orders in perturbation theory). To this end, let us first obtain an overview of the (classical) moduli space, i.e. the space of massless fields with flat potential parametrizing the vacua of the theory. Since there are no perturbative corrections to the superpotential, the moduli space is given in all orders of perturbation theory by the solutions of the $D$-terms for the gauge fields ${ }^{11}$. Finding supersymmetric solutions (i.e. minima of the $D$-term potential), it turns out, amounts to solving the equation

$$
\bar{\Phi} \Phi-\widetilde{\Phi} \overline{\widetilde{\Phi}}=0
$$

Employing suitable gauge and flavor rotations, this is solved by

$$
\langle\Phi\rangle=\langle\overline{\widetilde{\Phi}}\rangle=\left(\begin{array}{cccc}
v_{1} & & & \\
& v_{2} & & \\
& & \ddots & \\
& & & v_{f}
\end{array}\right)
$$

\footnotetext{
${ }^{11}$ And determining the non-perturbative superpotential amounts to looking for deviations from the classical moduli space.
} 
where the angles indicate that this is for field vevs. The matrix has the format $N_{f} \times N_{c}$. Small coupling of the theory translates to all vevs being large and we shall assume that this is the case.

Thus, at a generic point in moduli space (all $v_{i}$ distinct and of roughly the same magnitude) the gauge group is broken by the Higgs effect to $S U\left(N_{c}-N_{f}\right)$ and there remain $N_{f}^{2}$ light degrees of freedom which can be described by the meson matrix

$$
M_{f f^{\prime}}=\Phi_{f c} \widetilde{\Phi}_{c f^{\prime}}
$$

We can now look at the low energy theory corresponding to SQCD. If $N_{f}=N_{c}-1$, the gauge gauge group is completely broken and the only remaining light degrees of freedom are the meson fields $M_{f f^{\prime}}$.

If $N_{f}<N_{c}-1$ there is an unbroken gauge group $S U\left(N_{c}-N_{f}\right)$ with no matter fields transforming under the group at low energies. The theory then is believed to exhibit a mass gap (like in ordinary QCD) below which scale the only light fields are again the mesons $M_{f f^{\prime}}$. Thus, in both cases the low energy theory of SQCD is a theory having the meson matrix $M_{f f^{\prime}}$ as its degrees of freedom.

Using the non-anomalous symmetries we now further constrain the form of any would-be non-perturbative superpotential. What kind of functional form could such a superpotential have? In any case, the symmetries of the theory should be respected. First of all, the superpotential will be a function of the meson matrix $M_{f f^{\prime}}$ which already means that it is $S U\left(N_{c}\right)$ and $U(1)_{V}$ invariant. The only flavor invariant one can form from this is

$$
M=\operatorname{det}_{f f^{\prime}} M_{f f^{\prime}}=\operatorname{det} \Phi \widetilde{\Phi} .
$$

To make the superpotential $W$ invariant under the non-anomalous $U(1)_{R}$ it is then necessary that

$$
W \propto[\operatorname{det}(\Phi \widetilde{\Phi})]^{-\frac{1}{N_{c}-N_{f}}}
$$

On dimensional grounds, then one has

$$
W=\Lambda^{\frac{3 N_{c}-N_{f}}{N_{c}-N_{f}}}[\operatorname{det}(\Phi \widetilde{\Phi})]^{-\frac{1}{N_{c}-N_{f}}}
$$

which is the celebrated Affleck-Dine-Seiberg (ADS) superpotential. $\Lambda$ is the scale of the effective theory.

In the case $N_{f}<N_{c}-1$ this superpotential is generated by gaugino condensation. In the case $N_{f}=N_{c}-1$ it is generated by instantons as was shown by $\mathrm{ADS},{ }^{12}$ in which case the superpotential takes the characteristic form

$$
W=\Lambda^{2 N_{c}+1} \frac{1}{\operatorname{det}(\Phi \widetilde{\Phi})} .
$$

\footnotetext{
${ }^{12}$ The scale dependent prefactor is renormalization scheme dependent and was determined explicitly in [77, 75.
} 
A recapitulation of the instanton calculation leading to the ADS superpotential [5, 47, 75. would seriously lead us to far afield, as it requires a fair amount of formalism. One qualitative difference to the sort of calculation performed in section 2.3 is that we would be dealing with a supersymmetric gauge theory instanton, which has further, fermionic, collective coordinates.

A further remark is in order: In spontaneously broken gauge theories, i.e. in the presence of one or several Higgs fields, there are, in a strict sence, no instantons but fields of non-trivial topology still play a role in the path integral. In relation to the ADS superpotential we have precisely this situation and this is the reason why, in the literature, one finds the term 'constrained instanton' in this connection [3].

Let us reiterate: SQCD with $N_{f}$ flavors and $N_{c}$ colors for $N_{f}<N_{c}$ has no perturbative superpotential but develops a non-perturbative one. Thus, we have in our hands the quantum corrected moduli space. In the case $N_{f}=N_{c}-1$ the origin of the non-perturbative superpotential is a (constrained) instanton in SQCD. Of course, there will also be higherinstanton corrections but they will only give further small corrections. Note also that (2.63) is manifestly supersymmetry breaking. A natural thing to do then is to look for minima of the potential induced by the superpotential, i.e. to look for ground states of the quantum theory.

One can show that that the potential derived from the superpotential

$$
V=\sum_{f}\left(\left|\frac{\partial W}{\partial \Phi_{f}}\right|^{2}+\left|\frac{\partial W}{\partial \widetilde{\widetilde{\Phi}}_{f}}\right|^{2}\right)
$$

has no minimum. In fact, it tends to zero slope only for $\langle\operatorname{det} \Phi \widetilde{\Phi}\rangle \rightarrow \infty$, meaning in words that it drives the vevs of the fields to infinity. In this sense, SQCD with vevs seems to have no ground state. In the usual parlance one refers to such a situation as a runaway vacuum. However, there might be a loop-hole to this argument. Namely, it is conceivable that in the case $\operatorname{det} \Phi \widetilde{\Phi}=0$ wavefunction renormalization (which we have ignored) could produce new vacua (cf. the remarks in [139]). In any case, the 'naive' potential is depicted in figure 2.8 . 


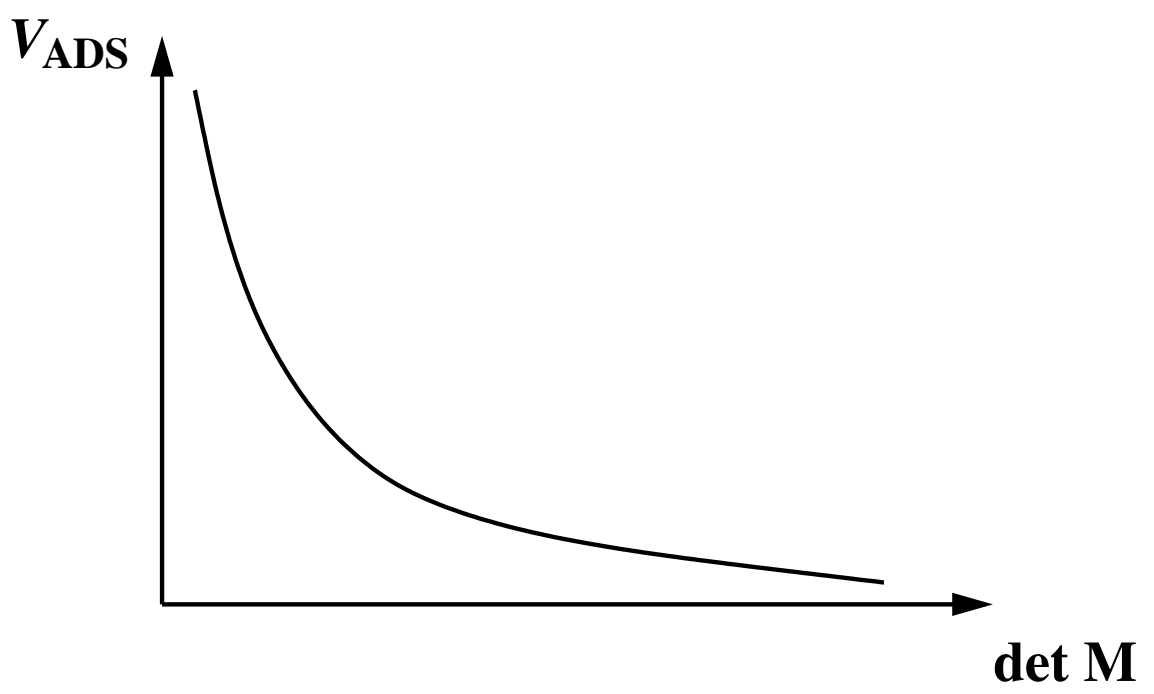

Figure 2.3: Due to the Affleck-Dine-Seiberg superpotential, SQCD exhibits a so-called 'runaway vaccum,' meaning that the ground state has infinite vev. Of course, this is not a vacuum at all. 


\section{Chapter 3}

\section{The ADS Superpotential from D-instantons ${ }^{1}$}

\footnotetext{
${ }^{1}$ This chapter is based, sometimes verbatim, on the paper 7 together with R. Blumenhagen, D. Lüst, E. Plauschinn, and M. Schmidt-Sommerfeld.
} 


\subsection{Introduction}

In this chapter we derive from a stringy point of view the Affleck-Dine-Seiberg (ADS) superpotential discussed in section 2.8. The upshot of section 2.8 was that in supersymmetric QCD (SQCD) with gauge group $S U\left(N_{c}\right)$ and $N_{f}=N_{c}-1$ flavors a non-perturbative superpotential

$$
W_{\mathrm{ADS}}=\frac{\Lambda^{2 N_{c}+1}}{\operatorname{det} \Phi \widetilde{\Phi}}
$$

is induced by instantons. Here, as in section 2.8, $\Phi$ denotes the $N_{f} \times N_{c}$ superquark matrix, wheras $\widetilde{\Phi}$ is its $N_{c} \times N_{f}$ 'anti' counterpart.

It is clear that the ADS superpotential will have to be present in any string theory realization of SQCD. Therefore, at first sight, such an exercise might seem moot. However, the fact of the matter is that this is not at all so. First of all, the original publication [7] served the purpose of checking the charged matter field coupling instanton calculus explicated in [34] in a nontrivial situation for which it was clear what result to expect (the ADS superpotential!). Second, as we shall see, the validity of (3.1) will depend on a particular limit in the string theory setup for SQCD, so that it is not clear that it is allowed to assume that (3.1) will also feature in other limits. Since in string theory model building quite often non-perturbative terms are added to the effective action by hand, this fact should be read as a warning sign.

We shall construct our stringy SQCD from a (local) intersecting D6-brane model on a Calabi-Yau threefold. The microscopic origin of the SQCD instanton will then be a socalled E2-instanton ${ }^{2}$ wrapping a three-cycle of the internal geometry and being pointlike in four dimensions. Using a slight extension of the charged matter field instanton calculus of [34] to incorporate also bosonic zero modes we then derive the Affleck-Dine-Seiberg superpotential directly from string theory, i.e. without any need to argue in terms of the effective field theory.

\subsection{Instanton Generated Superpotential}

Before we can construct a stringy realization of SQCD and then derive the ADS-superpotential microscopically as the effect of an E2-instanton in the model, we first have to explain the methods for doing so.

Thus, in this section we review and slightly extend the formalism developed in [34] for the computation of instanton contributions to the superpotential of type IIA orientifolds

\footnotetext{
${ }^{2}$ Some authors write $\mathrm{D} p$-instantons instead of $\mathrm{E} p$-instantons. This has the disadvantage that in diagrams they are not easily distinguished from $\mathrm{D} p$-branes. We shall generally stick with the denomination Ep-instanton, where E alludes to its Euclidean world-volume.
} 
with intersecting D6-branes, where the instanton effects are due to E2-instantons wrapping compact three-cycles of the internal Calabi-Yau geometry.

We take as given an intersecting D6-brane model preserving $\mathcal{N}=1$ supersymmetry in four dimensions, meaning that the brane stacks of the model wrap special Lagrangian three-cycles $\Pi_{a}$ in the compactification Calabi-Yau manifold, all preserving the same supersymmetry (for details on the model bulding rules used here, see [35]).

As mentioned, in such a model, spacetime instanton effects are induced by E2-instantons wrapping three-cycles $\Xi$ in the Calabi-Yau. From the four-dimensional perspective such an E2-instanton will look like a point. These instantons can contribute to the four-dimensional superpotential if they preserve half of the $\mathcal{N}=1$ supersymmetry. This condition translates to the statement that only E2-instantons wrapping special Lagrangian three-cycles will contribute to the superpotential and in the following we shall restrict our attention to them.

For E2-instantons invariant under the orientifold projection we then have four translational zero modes $x^{\mu}$, corresponding to the position of the instanton in four dimensions, and two fermionic zero modes $\theta^{\alpha}$. The fermionic zero modes correspond to the two four-dimensional supersymmetries broken by the instanton. If the E2-instanton is not invariant under the orientifold projection there are another two fermionic zero modes $\bar{\theta}^{\alpha}$, as it also breaks four of the eight supercharges in the bulk [86].

We require in the following that there do not arise any further zero modes from E2-E2 and E2-E2' open strings (the prime denotes the orientifold image), so that in particular the three-cycle $\Xi$ should be rigid, i.e. $b_{1}(\Xi)=0$.

Therefore, additional zero modes can only arise from the intersection of the instanton $\Xi$ with D6-branes $\Pi_{a}$. These zero modes are:

- There are always $N_{a}\left[\Xi \cap \Pi_{a}\right]^{+}$chiral fermionic zero modes $\lambda_{a, I}$ and $N_{a}\left[\Xi \cap \Pi_{a}\right]^{-}$ anti-chiral ones, $\widetilde{\lambda}_{a, J}:^{3}$

- At a smooth point in the Calabi-Yau moduli space there will typically be no bosonic zero modes coming from the E2-instanton. However, if the E2-instanton lies right on top of a D6-brane brane stack $a$, we gets $4 N_{a}$ bosonic zero modes. Let us organize them into two complex modes $b_{\alpha}$ with $\alpha=1,2$.

As we will see in section 3.3 , for the case that an E2-instanton wraps the same cycle as some brane stack, there can appear extra conditions, so-called ADHM constraints, on the zero modes arising from the flatness conditions for the effective zero-dimensional gauge theory on the E2-instanton. In this case, the above numbers only give the unrestricted number of zero modes. Put another way, the manifold parametrized by the zero modes need not have the same dimension as the sum of the numbers above.

\footnotetext{
${ }^{3}$ The physical intersection number $\Pi_{a} \cap \Pi_{b}$ between two cycles $\Pi_{a}$ and $\Pi_{b}$ is the sum of positive $\left[\Pi_{a} \cap \Pi_{b}\right]^{+}$and negative $\left[\Pi_{a} \cap \Pi_{b}\right]^{-}$intersections.
} 
In [29, 34] it was argued that each E2-D6 zero mode carries an extra normalization factor of $\sqrt{g_{s}}$ so that contributions to the superpotential can only arise from CFT disk and oneloop diagrams involving an E2-instanton as a boundary. Here the disk contains precisely two E2-instanton zero mode insertions and the one-loop diagram none. For details we refer to [34]. Here we only recall the final result for the E2-instanton contribution to the superpotential.

We first introduce the short-hand notation

$$
\widehat{\Phi}_{a_{k}, b_{k}}\left[\vec{x}_{k}\right]=\Phi_{a_{k}, x_{k, 1}} \cdot \Phi_{x_{k, 1}, x_{k, 2}} \cdot \Phi_{x_{k, 2}, x_{k, 3}} \cdot \ldots \cdot \Phi_{x_{k, n-1}, x_{k, n}} \cdot \Phi_{x_{k, n(k)}, b_{k}}
$$

for the chain-product of open string vertex operators, where we have defined $\widehat{\Phi}_{a_{k}, b_{k}}[\overrightarrow{0}]=$ $\Phi_{a_{k}, b_{k}}$.

The contribution of a single E2-instanton to the superpotential action is then determined by evaluating the following zero mode integral over disk and one-loop open string CFT amplitudes 4

$$
\begin{aligned}
S_{W}=\frac{1}{\ell_{s}^{3}} & \int d^{4} x d^{2} \theta \sum_{\text {conf. }} \prod_{I} d \lambda_{I} \prod_{J} d \widetilde{\lambda}_{J} \prod_{\alpha=1}^{2} \prod_{i} d b_{\alpha, i} d \bar{b}_{\alpha, i} \\
& \times \delta\left(\lambda, \widetilde{\lambda}, b_{\alpha}, \bar{b}_{\alpha}\right) \times \exp \left(\langle 1\rangle^{1-\text { loop }}\right) \times \exp \left(\langle 1\rangle^{\text {disk }}\right) \\
& \times \exp \left(\sum_{k}\left\langle\left\langle\Phi_{a_{k}, b_{k}}\left[\vec{x}_{k}\right]\right\rangle\right\rangle_{\lambda_{k} \widetilde{\lambda}_{k}}^{\text {disk }}\right) \times \exp \left(\sum_{l}\left\langle\left\langle\Phi_{a_{l}, b_{l}}\left[\vec{x}_{k}\right]\right\rangle\right\rangle_{b_{\alpha, l} \bar{b}_{\alpha, l}}^{\text {disk }}\right),
\end{aligned}
$$

where we work in the convention that all fields carry no scaling dimension and the sum is over all possible vertex operator insertions.

Note that in this formula the $\bar{\theta}^{\alpha}$ zero modes have already been integrated over leading to $\delta$-functions representing the fermionic ADHM constraints [18], which we will explain later. There are further $\delta$-functions involving only bosonic zero modes. These incorporate the bosonic ADHM constraints or, in more physical terms, the F-term and D-term constraints of the effective theory on the E2-instanton.

Since we will not attach any charged matter fields to the one-loop diagrams, compared to [34, we left out this term in (3.3). The prefactor $\ell_{s}^{-3}$ is chosen for dimensional reasons and is so far only determined up to numerical factors.

The vacuum disk amplitude is given by

$$
\langle 1\rangle^{\text {disk }}=-S_{\mathrm{E} 2}=-\frac{1}{g_{s}} \frac{V_{\mathrm{E} 2}}{\ell_{s}^{3}}=-\frac{8 \pi^{2}}{g_{\mathrm{YM}}^{2}},
$$

\footnotetext{
${ }^{4}$ In light of the developments in chapter 5 we should add that this formula is to be interpreted in such a way as to include only the holomorphic parts of the one-loop and disk-diagrams. In any case, it makes perfectly good sense in the field theory limit of section 3.3.1, as then the matter Kähler metrics (cf. chapter 5) can be thought of as giving trivial contributions.
} 
where the four-dimensional Yang-Mills gauge coupling is for the gauge theory on a stack of D6-branes wrapping the same internal three-cycle as the instanton.

In 34 the superpotential was given for the case without any additional charged bosonic zero modes $b_{\alpha}, \bar{b}_{\alpha}$. Since they also carry the extra normalization factor $\sqrt{g_{s}}$, precisely two of them can be attached to a disk diagram. Moreover, since they commute, the whole exponential term appears in the superpotential (3.3).

The one-loop contributions are annulus diagrams for open strings with one boundary on the E2-instanton and the other boundary on the various D6-brane stacks:

$$
\langle 1\rangle^{1-\mathrm{loop}}=\sum_{a}\left[Z^{\prime A}\left(\mathrm{E} 2, \mathrm{D} 6_{a}\right)+Z^{\prime A}\left(\mathrm{E} 2, \mathrm{D} 6_{a}^{\prime}\right)\right]+Z^{\prime M}(\mathrm{E} 2, \mathrm{O} 6)
$$

Here $Z^{\prime}$ means that we only sum over the massive open string states in the loop amplitude, as the zero modes are taken care of explicitly. We shall come back to these one-loop diagrams in chapter 5. In fact, we do not need their explicit form here but only need to know that they vanish in the field theory limit to be discussed in section 3.3.1.

The last ingredients to compute the rhs of equation (3.3), in principle at least, are the disk diagrams with insertion of matter fields $\Phi_{a, b}$ and fermionic or bosonic zero modes. In the case of inserting fermionic zero modes $\lambda$ and $\widetilde{\lambda}$, the explicit form of the disk diagram is

$$
\begin{aligned}
\left\langle\left\langle\Phi_{a_{k}, b_{k}}\left[\vec{x}_{k}\right]\right\rangle\right\rangle_{\lambda_{k} \widetilde{\lambda}_{k}}^{\text {disk }}= & = \\
& \int \frac{d z_{1} \ldots d z_{n+2}}{V_{1,2, n+2}}\left\langle V_{\lambda_{k}}\left(z_{1}\right) V_{\Phi_{a_{k}, x_{k_{1}}}}\left(z_{2}\right) \ldots V_{\Phi_{x_{k_{n}}, b_{k}}}\left(z_{n+1}\right) V_{\widetilde{\lambda}_{k}}\left(z_{n+2}\right)\right\rangle .
\end{aligned}
$$

For the disk diagram with insertion of bosonic zero modes $b_{\alpha}$ and $\bar{b}_{\alpha}$, one simply replaces $V_{\lambda_{k}}\left(z_{1}\right)$ by $V_{b_{\alpha, k}}\left(z_{1}\right)$ and $V_{\widetilde{\lambda}_{k}}\left(z_{n+2}\right)$ by $V_{\bar{b}_{\alpha, k}}\left(z_{n+2}\right)$.

In equation (3.6) the symbol $V_{i j k}$ denotes the measure

$$
V_{i j k}=\frac{d z_{i} d z_{j} d z_{k}}{\left(z_{i}-z_{j}\right)\left(z_{i}-z_{k}\right)\left(z_{j}-z_{k}\right)}
$$

with $z_{i} \rightarrow \infty, z_{j}=1$ and $z_{k}=0$. The remaining $z$ 's are to be ordered as $1 \geq z_{3} \geq z_{4} \geq$ $\ldots \geq z_{n+1} \geq 0$ and integrated over the interval $[0,1]$.

As expected, the stringy superpotential is entirely given in a semi-classical approximation. Still, it would be an impossible task to evaluate (3.3) exactly. Luckily, in section 3.3.1 we will see that in the field theory limit, only the leading order terms of the disk and one-loop amplitudes survive, considerably simplifying formula (3.3), thus making the integration over instantonic zero modes tractable. 


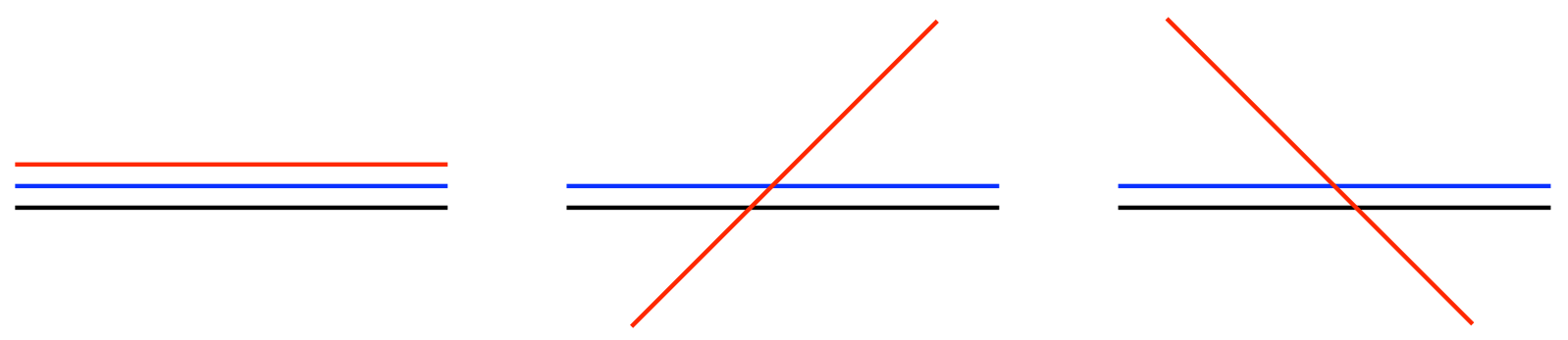

Figure 3.1: Intersecting D6-brane setup for SQCD for the case of a toroidal $T^{2} \times T^{2} \times T^{2}$ compactification. The color stack is shown in blue, the flavor stack is red. On top of the color stack is a single E2-instanton (black).

\subsection{SQCD from an Intersecting D6-brane Model}

We now construct SQCD as an intersecting D-brane model. As mentioned above, our starting point is type IIA string theory on a background of the form

$$
M_{4} \times X
$$

where $X$ is some Calabi-Yau manifold and $M_{4}$ is four-dimensional Minkowski-space. On this background we want to introduce D6-branes so as to give us SQCD in four dimensions at low energies.

To this end, we first wrap a stack of $N_{c}$ D6-branes around a special Lagrangian and rigid three-cycle $\Pi_{c} \subset X$, which we shall refer to as the color stack. The usual model building rules [35] then imply that the low energy effective action induced on the stack of branes will be $\mathcal{N}=1$ super Yang-Mills with gauge group $S U\left(N_{c}\right)$. This gauge theory must now be coupled to $N_{f}$ flavors of quarks $\Phi$ and anti-quarks $\widetilde{\Phi}$ transforming in the fundamental, respectively anti-fundamental representation of $S U\left(N_{c}\right)$. In intersecting D-brane models such matter fields can only come from bi-fundamental representations arising from the intersection of the color stack with a stack of $N_{f}$ branes, which we shall call the flavor stack, wrapping a special Lagrangian cycle $\Pi_{f} \neq \Pi_{c}$. Since we need non-chiral matter, we assume that the intersection number of $\Pi_{c}$ and $\Pi_{f}$ is non-chiral, i.e. that

$$
\left[\Pi_{c} \cap \Pi_{f}\right]^{ \pm}=1
$$

In our setup for SQCD the Yang-Mills instanton responsible for the Affleck-Dine-Seiberg superpotential will come from an E2-instanton lying on top of the color stack, i.e. wrapping the same internal cycle $\Pi_{c}$ as the color stack. Figure 3.1 illustrates how the brane setup we have arrived at could look like for a toroidal $T^{2} \times T^{2} \times T^{2}$ compactification. Later on (section 3.3.1) we shall take a field theory limit in order to decouple gravity and the gauge theory induced on the flavor stack. In this limit we do not need to worry about any orientifold planes which we would otherwise have to introduce in order to cancel charges 


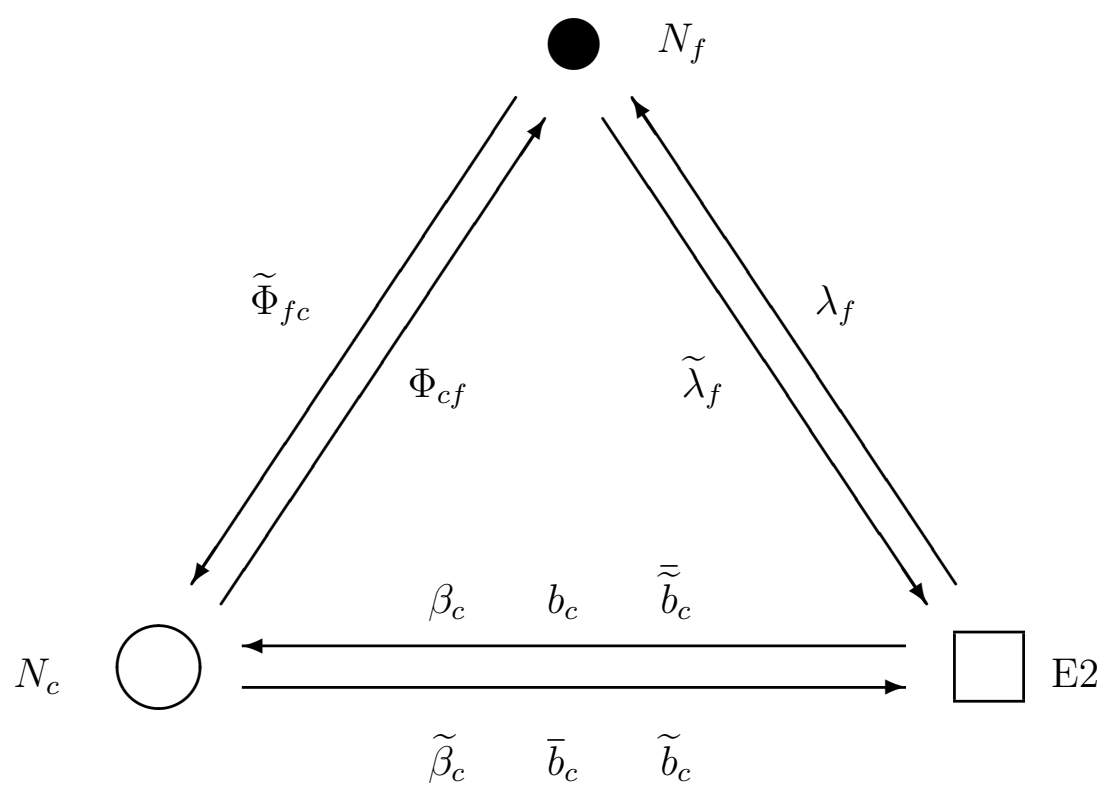

Figure 3.2: Extended quiver diagram for our local SQCD brane configuration showing the resulting zero modes. The circles represent the color, respectively flavor brane stacks, whereas the square denotes the E2-instanton. On the arrows are written the zero modes arising from the intersections of the various branes.

and tension of the color and flavor branes. In this respect we will have engineered a local model [96, 97] for SQCD.

Of prime importance for our purposes are the various massless fields arising in the (local) model we have constructed. These zero modes are summarized in figure 3.2, a so-called extended quiver diagram. First we have the quarks $\Phi_{c f}$ and anti-quarks $\Phi_{f c}$ arising from the intersection of the color and flavor brane stacks. They are the two 'parts' of the bi-fundamental matter arising at the intersection. Moreover, the quarks and anti-quarks are coupled to $S U\left(N_{c}\right)$ gauge theory in the required representations and are subject to a global $S U\left(N_{f}\right)$ flavor symmetry (at least in the field theory limit to be discussed below in section 3.3.1). There are also a number of zero modes arising from the intersections of the E2-instanton with the brane stacks which we shall now analyze in turn.

E2-E2: Since the E2-instanton wraps the same rigid three-cycle as the $\mathrm{D} 6_{c}$ brane stack, the massless E2-E2 modes are the four positions in Minkowski space $x^{\mu}$ with $\mu=0, \ldots, 3$ and four massless fermions $\theta^{\alpha}, \bar{\theta}^{\alpha}, \alpha=1,2$. The latter correspond to the breaking of four of the eight supercharges preserved in type IIA theory on a Calabi-Yau manifold. Two of these, namely the $\theta^{\alpha}$, are related to the two supersymmetries preserved by the D6-branes, but broken by the E2-instanton, and, together with the four bosons $x^{\mu}$ contribute the 
measure

$$
\int d^{4} x d^{2} \theta
$$

to the instanton induced superpotential action. Integrating the other two zero modes $\bar{\theta}^{\alpha}$ leads to the fermionic ADHM constraints as we discuss below.

E2-D6 $\mathbf{6}_{c}$ : Since the E2-instanton and the D6 $_{c}$ branes wrap the same three-cycle, they share three directions in the internal space and have N-D boundary conditions along Minkowski space. Therefore, the ground state energy in both the NS- and the R-sector is $E_{\mathrm{NS}, \mathrm{R}}=0$ and we get $N_{c}$ complete hypermultiplets of massless modes.

However, not all of these bosonic zero modes are independent. In fact as analyzed in detail in [29], the effective zero-dimensional supersymmetric gauge theory on the E2-instanton gives rise to D-term and F-term constraints, which in the field theory limit are nothing else than the ADHM constraints [69].

If we rename the complex bosonic zero modes $b_{\alpha, i}$ as $b_{1, i}=b_{i}$ and $b_{2, i}=\overline{\widetilde{b}}_{i}$, these constraints become 98.

$$
\sum_{c=1}^{N_{c}}\left[b_{c} \widetilde{b}_{c}+\bar{b}_{c} \overline{\widetilde{b}}_{c}\right]=0, \quad \sum_{c=1}^{N_{c}}\left[b_{c} \widetilde{b}_{c}-\bar{b}_{c} \overline{\widetilde{b}}_{c}\right]=0, \quad \sum_{c=1}^{N_{c}}\left[b_{c} \bar{b}_{c}-\widetilde{b}_{c} \overline{\widetilde{b}}_{c}\right]=0 .
$$

The first two can be interpreted as the F-term constraints Re $b_{c} \widetilde{b}_{c}=0$ and $\operatorname{Im} b_{c} \widetilde{b}_{c}=0$, the last one as the D-term constraint $\sum_{c=1}^{N_{c}}\left|b_{c}\right|^{2}-\left|\widetilde{b}_{c}\right|^{2}=0$.

We only have $2 N_{c}$ fermionic zero modes. However, in [29] it was shown in the T-dual picture that they have to satisfy the ADHM constraints

$$
\sum_{c=1}^{N_{c}}\left[\widetilde{\beta}_{c} b_{c}+\beta_{c} \widetilde{b}_{c}\right]=0 \quad \text { and } \quad \sum_{c=1}^{N_{c}}\left[\widetilde{\beta}_{c} \overline{\widetilde{b}}_{c}-\beta_{c} \bar{b}_{c}\right]=0
$$

These constraints can be recovered in the present setup by performing the integration over the aforementioned zero modes $\bar{\theta}^{\alpha}$ explicitly [29, 15]. More concretely, one can absorb the instanton zero modes with terms such as $\tilde{\beta}_{c} b_{c} \bar{\theta}$ and integrate over $\bar{\theta}$ to arrive at a $\delta$-function realisation of 3.12 .

All these constraints, i.e. equations (3.11) and (3.12), have to be implemented in the general formula for the superpotential (3.3). We will not perform the $\bar{\theta}$ integration explicitly, but realize all the constraints by the means of delta functions. 
E2-D6 : $_{f}$ In this sector, the ground state energy in the NS sector is positive, so that there are no bosonic zero modes. We only gets $N_{f}$ pairs of non-chiral zero modes $\lambda_{f}, \widetilde{\lambda}_{f}$, yielding the measure

$$
\int \prod_{f=1}^{N_{f}} d \lambda_{f} d \widetilde{\lambda}_{f}
$$

To summarize, for our SQCD setup the total integration measure for the computation of the E2-instanton generated superpotential (3.3) reads

$$
\begin{aligned}
\int d^{4} x d^{2} \theta \prod_{c=1}^{N_{c}} d b_{c} d \bar{b}_{c} d \widetilde{b}_{c} d \overline{\widetilde{b}}_{c} d \beta_{c} d \bar{\beta}_{c} \prod_{f=1}^{N_{f}} d \lambda_{f} d \widetilde{\lambda}_{f} \delta^{F}\left(\widetilde{\beta}_{c} b_{c}+\beta_{c} \widetilde{b}_{c}\right) \\
\delta^{F}\left(\widetilde{\beta}_{c} \overline{\widetilde{b}}_{c}-\beta_{c} \bar{b}_{c}\right) \delta^{B}\left(b_{c} \widetilde{b}_{c}+\bar{b}_{c} \overline{\widetilde{b}}_{c}\right) \delta^{B}\left(b_{c} \widetilde{b}_{c}-\bar{b}_{c} \overline{\widetilde{b}}_{c}\right) \delta^{B}\left(b_{c} \bar{b}_{c}-\widetilde{b}_{c} \overline{\widetilde{b}}_{c}\right),
\end{aligned}
$$

where in the two fermionic and three bosonic ADHM constraints summation over the color index is understood.

\subsubsection{Field Theory Limit}

Now let us discuss the field theory limit already mentioned several times in more detail. We have a stack of color D6-branes wrapping a three-cycle of size

$$
v_{c}=\frac{V_{c}}{\ell_{s}^{3}} \cong \frac{R_{c}^{3}}{\left(\alpha^{\prime}\right)^{\frac{3}{2}}}=r_{c}^{3}
$$

in string units. The flavor branes wrap a different cycle with volume $v_{f}=r_{c} r_{T}^{2}$, where $T$ stands for transversal. We are thinking here of the situation on the six-torus, where the flavor- and the color-cycle share one common direction, see figure 3.3 . The volume of the entire Calabi-Yau in string units is $v_{\mathrm{CY}} \cong r_{c}^{3} r_{T}^{3}$ and we have two dimensionless scales $r_{c}, r_{T}$ and one length scale $\sqrt{\alpha^{\prime}}$.

The effective four-dimensional couplings in our configuration are the Yang-Mills coupling of the color branes

$$
\frac{4 \pi}{g_{c}^{2}}=\frac{1}{g_{s}} \frac{V_{c}}{\ell_{s}^{3}} \cong \frac{r_{c}^{3}}{g_{s}}
$$

the Yang-Mills coupling of the flavor branes

$$
\frac{4 \pi}{g_{f}^{2}}=\frac{1}{g_{s}} \frac{V_{f}}{\ell_{s}^{3}} \cong \frac{r_{c} r_{T}^{2}}{g_{s}},
$$



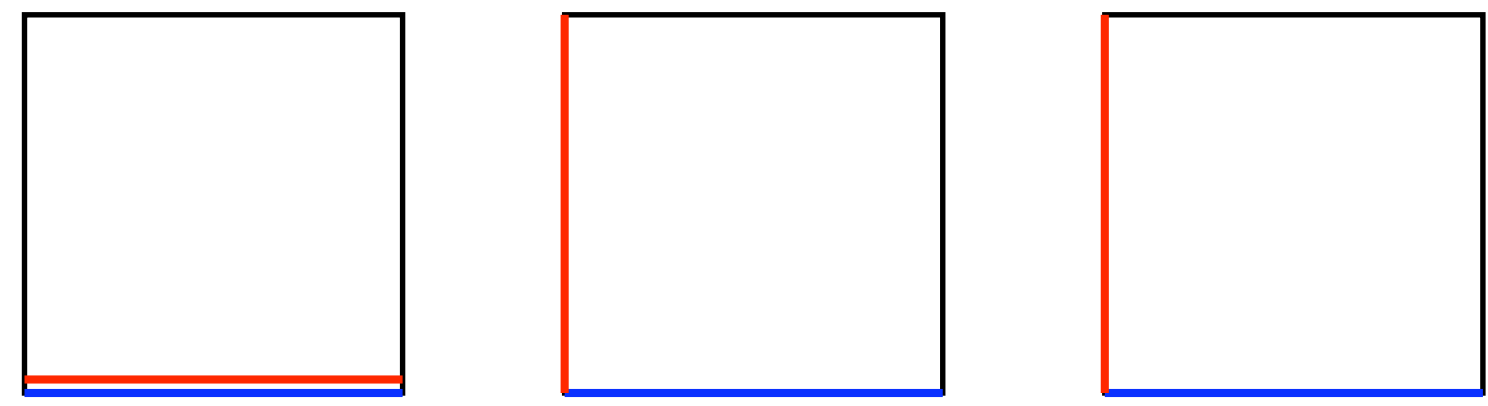

Figure 3.3: Cartoon of the intersection of the color (blue) and flavor cycles (red) in the compactification manifold.

and the Planck mass

$$
M_{P}^{2} \cong \frac{1}{\left(2 \pi \alpha^{\prime}\right) g_{s}^{2}} r_{c}^{3} r_{T}^{3}
$$

Clearly, the gauge coupling of the color branes should be fixed at finite $g_{c}$. In order for the Dirac-Born-Infeld theory on these branes to reduce to the Yang-Mills Lagrangian we have to suppress all higher $\alpha^{\prime}$ corrections, which means we have to take the $\alpha^{\prime} \rightarrow 0$ limit.

On the other hand, the gauge theory on the flavor branes should decouple from the dynamics, which means that $g_{f} \rightarrow 0$ or, in other words, that the transverse space of volume $v_{T}=r_{T}^{3}$ should decompactify, i.e. $r_{T} \rightarrow \infty$. In this limit, also $M_{P} \rightarrow \infty$ so that gravity decouples as well.

Further, in this limit the world-sheet instantons, giving stringy corrections to the disk correlators, scale like

$$
\exp \left(-\frac{J}{\alpha^{\prime}}\right) \simeq \exp \left(-r_{T}^{2}\right) \rightarrow 0
$$

so that only trivial instantons of zero size contribute to the field theory amplitude. Moreover, in (3.5) all the massive string modes decouple in the $\alpha^{\prime} \rightarrow 0$ limit.

The same holds for potential Kaluza-Klein and winding modes in the various E2-D6 brane sectors, as their masses scale like

$$
M_{\mathrm{KK}}^{2}=\frac{1}{R_{c}^{2}} \simeq \frac{1}{\alpha^{\prime} r_{c}^{2}} \rightarrow \infty \quad \text { and } \quad M_{\mathrm{wind}}^{2}=\frac{R_{f}^{2}}{\alpha^{\prime 2}} \simeq \frac{r_{T}^{2}}{\alpha^{\prime}} \rightarrow \infty .
$$

The gauge coupling of the effective zero-dimensional gauge theory on the E2-instanton reads

$$
\frac{4 \pi}{g_{E}^{2}}=\frac{\ell_{s}^{4}}{g_{s}} \frac{V_{3}}{\ell_{s}^{3}} \simeq \frac{\alpha^{\prime 2} r_{c}^{3}}{g_{s}} \rightarrow 0
$$


i.e. naively, in the field theory limit the effective theory on the instanton decouples. However, this theory should provide the ADHM constraints, so that it better not decouple completely. In fact, as shown in [29], the operators up to dimension four in the E2-instanton action survive precisely due to extra factors of $\sqrt{g_{E}} \simeq \sqrt{g_{s}}$ in the vertex operators for the E2-D6 modes.

To summarize, in the field theory limit,

$$
\alpha^{\prime} \rightarrow 0, \quad v_{T} \rightarrow \infty, \quad v_{c}=\text { finite }
$$

the complete string theory formula $(3.3)$ simplifies dramatically with only a few disk diagrams surviving. All higher order $\alpha^{\prime}$ corrections in the matter fields are suppressed and the effective theory on the E2-instanton provides the ADHM constraints for the massless E2-D6 ${ }_{c}$ open string modes.

\subsubsection{Disk Amplitudes}

In order to evaluate the superpotential (3.3) for our locally engineered configuration of intersecting D6-branes, it remains to compute the appearing disk diagrams with two fermionic, respectively bosonic zero modes attached. Looking at the extended quiver diagram in figure 3.2, we realize that, due to the non-chirality of SQCD, numerous insertions of chains of matter fields $\Phi$ and $\widetilde{\Phi}$ are possible. However, in the field theory limit only the leading order term with the minimal number of $\Phi$ and $\widetilde{\Phi}$ insertions survives.

In this section we compute these one- and two-point CFT disk amplitudes with two fermionic or bosonic zero modes in the background. To eventually compare our result to field theory, we first have to ensure that the string amplitudes and the vertex operators are normalized correctly.

Here we will explicitly take care of factors of $g_{s}, \alpha^{\prime}$ and $v_{c}$. As we will see at the end of the computation, additional (often convention dependent) numerical factors can always be absorbed into the definition of the dynamically generated scale $\Lambda$ and are therefore not taken care of explicitly.

\section{Normalization}

In general, conformal field theory (disk) amplitudes carry a normalization factor $C$, i.e.

$$
\left\langle\left\langle\prod_{k=1}^{K} \Phi_{k}\right\rangle\right\rangle=C \int \frac{d z_{1} \ldots d z_{K}}{V_{1,2, K}}\left\langle V_{\Phi_{1}}\left(z_{1}\right) V_{\Phi_{2}}\left(z_{2}\right) \ldots V_{\Phi_{K}}\left(z_{K}\right)\right\rangle,
$$

where $C$ depends on the boundaries of the disk. If there is only a single boundary brane $\mathrm{D} p$, then $C$ is nothing else than the tension of the $\mathrm{D} p$-brane

$$
C=\frac{\mu_{p}}{g_{s}}=\frac{2 \pi}{g_{s} \ell_{s}^{p+1}} .
$$




\begin{tabular}{c||c|c|c|c|c|c|c} 
Branes & $\mathrm{D} 6_{c}$ & $\mathrm{D} 6_{f}$ & $\mathrm{D} 6_{c}, \mathrm{D} 6_{f}$ & $\mathrm{E} 2$ & $\mathrm{E} 2, \mathrm{D} 6_{c}$ & $\mathrm{E} 2, \mathrm{D} 6_{f}$ & $\mathrm{E} 2, \mathrm{D} 6_{c}, \mathrm{D} 6_{f}$ \\
\hline$C$ & $\frac{2 \pi v_{c}}{g_{s} \ell_{s}^{4}}$ & $\frac{2 \pi v_{f}}{g_{s} \ell_{s}^{4}}$ & $\frac{2 \pi}{g_{s} \ell_{s}^{4}}$ & $\frac{2 \pi v_{c}}{g_{s}}$ & $\frac{2 \pi v_{c}}{g_{s}}$ & $\frac{2 \pi}{g_{s}}$ & $\frac{2 \pi}{g_{s}}$
\end{tabular}

Table 3.1: Disk normalization factors $C$ for the various combinations of possible boundaries.

However, if the disk has more boundaries, then the normalization is given by the common sub-locus of all boundaries. For the branes D6 ${ }_{c}, \mathrm{D} 6_{f}$ and E2 the resulting normalization factors are shown in table 3.1

Having fixed the normalization factors of the disk, we have to take care of the normalization of the vertex operators. These can be fixed by comparison with the standard normalization in field theory.

Let us first consider the vertex operators for the gauge boson in the (-1)-ghost picture

$$
V_{A}^{(-1)}(z)=A^{\mu} e^{-\varphi(z)} \psi_{\mu}(z) e^{i p \cdot X(z)} .
$$

In this normalization, the spacetime field $A^{\mu}$ is dimensionless. The standard field theory normalization is then obtained by scaling $A^{\mu}=\left(2 \pi \alpha^{\prime}\right)^{\frac{1}{2}} A_{\text {phys }}^{\mu}$. The Yang-Mills gauge coupling at the string scale is given by

$$
\frac{1}{g_{\mathrm{YM}}^{2}}=\frac{1}{2} C_{\mathrm{D} 6_{c}}\left(2 \pi \alpha^{\prime}\right)^{2}
$$

In order not to overload the notation with too many scaling factors, in the following we first normalize the vertex operators such that the spacetime fields do not carry any scaling dimension. Only in the very end we move to physical fields by introducing appropriate $\left(2 \pi \alpha^{\prime}\right)^{\Delta / 2}$ factors, where $\Delta$ denotes the four-dimensional scaling dimension of the field.

Next, we have to normalize the matter fields localized on the intersection of the two D6branes. We want the kinetic term to be normalized as in the work by Affleck, Dine, and Seiberg

$$
S_{\text {matter }}=\int d x^{4} \frac{1}{g_{\mathrm{YM}}^{2}} \partial_{\mu} \Phi \partial^{\mu} \bar{\Phi}
$$

Since now the disk normalization does not contain the volume $v_{c}$, we have to put this factor in the normalization of the vertex operators, leading to

$$
V_{\Phi}^{(-1)}(z)=\sqrt{v_{c}} \Phi e^{-\varphi(z)} \Sigma_{1 / 2}^{c f}(z) e^{i p \cdot X(z)},
$$

where $\Sigma_{1 / 2}^{c f}$ is an internal twist operator of conformal dimension $h=1 / 2$.

As explained in section 3.2 , for the instanton zero modes not to decouple in the field theory limit, their vertex operators must contain extra factors of $\sqrt{g_{s}}$. This leads to the following 
form of the vertex operator for the E2-D6 $6_{c}$ bosonic zero modes $b_{c}$

$$
V_{b_{c}}^{(-1)}(z)=\sqrt{\frac{g_{s}}{2 \pi v_{c}}} b_{c} e^{-\varphi(z)} \prod_{\mu=0}^{3} \sigma_{\frac{1}{16}}^{\mu}(z) \prod_{\nu=0}^{3} s_{\frac{1}{16}}^{\nu}(z)
$$

where the $\sigma_{\frac{1}{16}}^{\mu}(z)$ denote the bosonic twist fields of conformal dimension $h=1 / 16$ and $s_{\frac{1}{16}}^{\nu}(z)$ the fermionic ones of the same conformal dimension.

Similarly, for the fermionic instanton zero modes in this sector we write

$$
V_{\beta_{c}}^{(-1 / 2)}(z)=\sqrt{\frac{g_{s}}{2 \pi v_{c}}} \beta_{c} e^{-\frac{\varphi(z)}{2}} \prod_{\mu=0}^{3} \sigma_{\frac{1}{16}}^{\mu}(z) \Sigma_{3 / 8}^{c}(z)
$$

Taking into account the different disk normalizations for the charged matter fermionic zero modes, the vertex operator is

$$
V_{\lambda_{f}}^{(-1 / 2)}(z)=\sqrt{\frac{g_{s}}{2 \pi}} \lambda_{f} e^{-\frac{\varphi(z)}{2}} \prod_{\mu=0}^{3} \sigma_{\frac{1}{16}}^{\mu}(z) \Sigma_{3 / 8}^{f}(z)
$$

In the fermionic vertex operators, $\Sigma_{3 / 8}$ denote appropriate Ramond fields, whose form depends on the concrete CFT description of the internal Calabi-Yau manifold.

\section{Three-point amplitudes}

As we mentioned earlier, in the field theory limit (3.22) the computation of the single instanton generated superpotential (3.3) simplifies considerably. In particular, for our setup shown in figure 3.2 we are left with only two disk diagrams with insertion of two fermionic zero modes from the set $\beta_{c}, \widetilde{\beta}_{c}, \lambda_{f}, \widetilde{\lambda}_{f}$. See for instance [48, 152, 2, 109, 26] for the conformal field theory computation of disk amplitudes for intersecting D-brane models. The appropriate combination of these zero modes can be obtained by looking at the extended quiver diagram in figure 3.2

However, because of $U(1)$ charge cancellation on the world-sheet, the fermionic zero modes couple to anti-holomorphic matter fields. The explicit form of the first disk correlator is

$$
\left\langle\left\langle\widetilde{\Phi}_{c f}\right\rangle\right\rangle_{\beta_{c} \widetilde{\lambda}_{f}}^{\text {disk }}=\frac{2 \pi}{g_{s}} \int \frac{d z_{1} d z_{2} d z_{3}}{V_{123}}\left\langle V_{\beta_{c}}^{(-1 / 2)}\left(z_{1}\right) V_{\widetilde{\Phi}_{c f}}^{(-1)}\left(z_{2}\right) V_{\widetilde{\lambda}_{f}}^{(-1 / 2)}\left(z_{3}\right)\right\rangle .
$$

The second disk correlator is $\left\langle\left\langle\bar{\Phi}_{f c}\right\rangle\right\rangle_{\lambda_{f} \widetilde{\beta}_{c}}^{\text {disk }}$ and has a form analogous to the first one. 
The evaluation of these two disk correlators is easily achieved using the following threepoint functions

$$
\begin{aligned}
\left\langle\sigma_{\frac{1}{16}}^{\mu}\left(z_{1}\right) \sigma_{\frac{1}{16}}^{\nu}\left(z_{3}\right)\right\rangle & =\frac{\delta^{\mu \nu}}{\left(z_{1}-z_{3}\right)^{\frac{1}{8}}}, \\
\left\langle e^{-\frac{\varphi\left(z_{1}\right)}{2}} e^{-\varphi\left(z_{2}\right)} e^{-\frac{\varphi\left(z_{3}\right)}{2}}\right\rangle & =\frac{1}{\left(z_{1}-z_{2}\right)^{\frac{1}{2}}\left(z_{1}-z_{3}\right)^{\frac{1}{4}}\left(z_{2}-z_{3}\right)^{\frac{1}{2}}}, \\
\left\langle\Sigma_{\frac{3}{8}}^{c}\left(z_{1}\right) \overline{\widetilde{\Sigma}}_{\frac{1}{2}}^{c f}\left(z_{2}\right) \Sigma_{\frac{3}{8}}^{f}\left(z_{3}\right)\right\rangle & =\frac{1}{\left(z_{1}-z_{2}\right)^{\frac{1}{2}}\left(z_{1}-z_{3}\right)^{\frac{1}{4}}\left(z_{2}-z_{3}\right)^{\frac{1}{2}}},
\end{aligned}
$$

and we directly obtain the effective term in 3.3

$$
\mathcal{L}_{\mathrm{ferm}}=\beta_{c} \overline{\widetilde{\Phi}}_{c f} \widetilde{\lambda}_{f}+\lambda_{f} \bar{\Phi}_{f c} \widetilde{\beta}_{c}
$$

Note that this is the same Lagrangian as appears in the field theory calculation [70].

\section{Four-point amplitude}

In the field theory limit we have to compute (essentially) only one disk amplitude with insertion of two bosonic zero modes. Looking again at the extended quiver in figure 3.2 , we are led to insert the bosonic zero modes $\{b, \bar{b}\}$ and $\{\overline{\widetilde{b}}, \widetilde{b}\}$.

Because of $U(1)$ charge cancellation on the world-sheet, the matter fields can only be paired as $\Phi \bar{\Phi}$ and as $\widetilde{\Phi} \widetilde{\Phi}$. The explicit form of the CFT four-point function is

$$
\left\langle\left\langle\Phi_{c f} \bar{\Phi}_{f c^{\prime}}\right\rangle\right\rangle_{b_{c} \bar{b}_{c^{\prime}}}^{\text {disk }}=\frac{2 \pi}{g_{s}} \int \frac{d z_{1} d z_{2} d z_{3} d z_{4}}{V_{124}}\left\langle V_{b_{c}}^{(-1)}\left(z_{1}\right) V_{\Phi_{c f}}^{(0)}\left(z_{2}\right) V_{\bar{\Phi}_{f c^{\prime}}}^{(0)}\left(z_{3}\right) V_{\bar{b}_{c^{\prime}}}^{(-1)}\left(z_{4}\right)\right\rangle
$$

and similarly for $\{\overline{\widetilde{b}} \widetilde{b}\}$ and $\widetilde{\bar{\Phi}} \widetilde{\Phi}$.

The vertex operators for the bosons of the matter superfields in the (0)-ghost picture are given by

$$
V_{\Phi}^{(0)}(z)=\sqrt{v_{c}} \Phi\left(\Sigma_{1}(z)+i(p \cdot \psi) \Sigma_{1 / 2}(z)\right) e^{i p \cdot X(z)},
$$

where $\Sigma_{1}(z)=\left\{G_{-1 / 2}, \Sigma_{1 / 2}(z)\right\}$ is an internal operator of conformal dimension $h=1$.

We shall forgo the explicit calculation of the correlator here, but only note the effective term in (3.3) originating from bosonic zero modes to be [7]

$$
\mathcal{L}_{\text {bos }}=\frac{1}{2} b_{c}\left(\Phi_{c f} \bar{\Phi}_{f c^{\prime}}+\widetilde{\Phi}_{c f} \widetilde{\Phi}_{f c^{\prime}}\right) \bar{b}_{c^{\prime}}+\frac{1}{2} \overline{\widetilde{b}}_{c}\left(\Phi_{c f} \bar{\Phi}_{f c^{\prime}}+\widetilde{\Phi}_{c f} \widetilde{\Phi}_{f c^{\prime}}\right) \widetilde{b}_{c^{\prime}}
$$

This again agrees with the field theory Lagrangian [70]. 


\subsection{Calculating the ADS Superpotential}

Having engineered a local intersecting D6-brane model giving rise to $S U\left(N_{c}\right)$ super YangMills theory with $N_{f}$ non-chiral flavors and having determined the leading order disk diagrams, we now come to the evaluation of the zero mode integrals in (3.3) in order to recover the Affleck-Dine-Seiberg superpotential

$$
W=\frac{\Lambda^{2 N_{c}+1}}{\operatorname{det}\left(M_{f f^{\prime}}\right)}
$$

Note that here the matter fields carry their canonical scaling dimension $\Delta=1$.

The dynamically generated scale $\Lambda$ is defined as

$$
\left(\frac{\Lambda}{\mu}\right)^{3 N_{c}-N_{f}}=\exp \left(-\frac{8 \pi^{2}}{g_{c}^{2}(\mu)}\right) .
$$

\subsubsection{Fermionic Zero Mode Integration}

In this subsection we compute to lowest order in $\alpha^{\prime}$ the contribution of the fermionic zero modes to the E2-instanton generated superpotential (3.3). The relevant disk diagrams have been computed in section 3.3 .2 .

However, since we will perform an integration over instanton zero modes, we should replace the matter fields $\Phi_{c f}$ by their vevs $\left\langle\Phi_{c f}\right\rangle$ and similarly for $\widetilde{\Phi}_{f c}$ and their conjugates. Furthermore, we have to satisfy the D-term constraint for the matter fields to ensure the supersymmetry of our setup so that we can apply formula 3.3 . Specifically, the D-term constraint implies (cf. (2.58))

$$
\left\langle\Phi_{c f}\right\rangle=\left\langle\widetilde{\Phi}_{c f}\right\rangle \quad \text { and } \quad\left\langle\widetilde{\Phi}_{c f}\right\rangle=\left\langle\bar{\Phi}_{c f}\right\rangle .
$$

Using these relations, we can rewrite (3.34) to obtain the relevant three-point couplings

$$
\beta_{c}\left\langle\Phi_{c f}\right\rangle \widetilde{\lambda}_{f}+\lambda_{f}\left\langle\widetilde{\Phi}_{f c}\right\rangle \widetilde{\beta}_{c}
$$

where $c=1, \ldots, N_{c}$ and $f=1, \ldots, N_{f}$. The sum over repeated indices is understood and in the following we will omit the vev brackets for ease of notation.

The next step is to implement the fermionic ADHM constraints 3.12 in terms of deltafunctions. For Grassmann-variables they read

$$
\delta\left(\widetilde{\beta}_{c_{1}} b_{c_{1}}+\beta_{c_{2}} \widetilde{b}_{c_{2}}\right) \delta\left(\widetilde{\beta}_{c_{3}} \overline{\widetilde{b}}_{c_{3}}-\beta_{c_{4}} \bar{b}_{c_{4}}\right)=\left(\widetilde{\beta}_{c_{1}} b_{c_{1}}+\beta_{c_{2}} \widetilde{b}_{c_{2}}\right)\left(\widetilde{\beta}_{c_{3}} \overline{\widetilde{b}}_{c_{3}}-\beta_{c_{4}} \bar{b}_{c_{4}}\right)
$$


Taking also into account the fermionic part of the integration measure (3.14), we arrive at the following expression for the fermionic contribution to the superpotential

$$
\begin{aligned}
I_{\text {ferm }}(\Phi, \widetilde{\Phi}, b, \widetilde{b})=\int \prod_{c=1}^{N_{c}} d \beta_{c} d \widetilde{\beta}_{c} \prod_{f=1}^{N_{f}} d \lambda_{f} d \widetilde{\lambda}_{f}\left(\widetilde{\beta}_{c_{1}} b_{c_{1}} \overline{\widetilde{b}}_{c_{2}} \widetilde{\beta}_{c_{2}}-\widetilde{\beta}_{c_{1}} b_{c_{1}} \bar{b}_{c_{2}} \beta_{c_{2}}\right. \\
\left.+\beta_{c_{1}} \widetilde{b}_{c_{1}} \overline{\widetilde{b}}_{c_{2}} \widetilde{\beta}_{c_{2}}-\beta_{c_{1}} \widetilde{b}_{c_{1}} \bar{b}_{c_{2}} \beta_{c_{2}}\right) \exp \left(\beta_{c} \Phi_{c f} \widetilde{\lambda}_{f}+\lambda_{f} \widetilde{\Phi}_{f c} \widetilde{\beta}_{c}\right)
\end{aligned}
$$

Note the dependence not only on the matter fields but also on the bosonic zero modes $b, \widetilde{b}$.

The fermionic modes $\beta$ and $\widetilde{\beta}$ in front of the exponent determine which part in the series expansion is picked out by the integral. For instance, in the case of a prefactor $\widetilde{\beta} \beta$ only $\left(\beta_{c} \Phi_{c f} \widetilde{\lambda}_{f}\right)^{N_{c}-1}$ and $\left(\lambda_{f} \widetilde{\Phi}_{f c} \widetilde{\beta}_{c}\right)^{N_{c}-1}$ survive the $d \beta$ integration. This implies, however, that the integral (3.43) can be non-zero only if

$$
N_{f}=N_{c}-1
$$

One can check that for prefactors $\beta \beta$ and $\widetilde{\beta} \widetilde{\beta}$ the complete fermionic integral vanishes, since the number of modes $\lambda$ is equal the number of modes $\widetilde{\lambda}$. Therefore, we have recovered nicely the constraint of Affleck, Dine and Seiberg that the non-perturbative superpotential of SQCD is only generated by an instanton for $N_{f}=N_{c}-1$.

By differentiating under the integral sign, and using some of the formulas of appendix $\mathrm{D}$, we find that the final result for the fermionic zero mode integration is

$$
I_{\text {ferm }}(\Phi, \widetilde{\Phi}, b, \widetilde{b})=\sum_{p, q=1}^{N_{c}}(-1)^{p+q}\left(b_{p} \bar{b}_{q}+\overline{\widetilde{b}}_{p} \widetilde{b}_{q}\right) \operatorname{det}\left[\left.\Phi \widetilde{\Phi}\right|_{q, p}\right] .
$$

Here, and in the following, $\Phi$ is the $N_{c} \times\left(N_{c}-1\right)$ matrix with elements $\Phi_{c, f}$ and similarly $\widetilde{\Phi}$ is an $\left(N_{c}-1\right) \times N_{c}$ matrix. The symbol $\left.A\right|_{q, p}$ denotes the matrix obtained from $A$ by deleting the $q$ 'th row and $p^{\prime}$ th column. The $(q, p)^{\prime}$ th element of a matrix $A$ will be denoted by $A_{q, p}$.

\subsubsection{Bosonic Zero Mode Integration}

We now turn to the evaluation of the bosonic zero mode integrals in (3.14). The necessary disk diagrams have been computed in section 3.3 .2 .

Following the same reasoning as for the three-point amplitudes, we replace the matter fields by their vevs and use the D-term constraint (3.40) to arrive at the four-point couplings

$$
b_{c}\left\langle\Phi_{c f}\right\rangle\left\langle\widetilde{\Phi}_{f c^{\prime}}\right\rangle \bar{b}_{c^{\prime}}+\overline{\widetilde{b}}_{c}\left\langle\Phi_{c f}\right\rangle\left\langle\widetilde{\Phi}_{f c^{\prime}}\right\rangle \widetilde{b}_{c^{\prime}}
$$


Note that in the following we will again omit the vev brackets for ease of notation. The ADHM constraints for the bosonic moduli space, or equivalently the D- and F-term constraints for the E2-instanton, are given in equation (3.11). Again, these have to be implemented as delta-functions in the integration measure (3.14).

The explicit form of the bosonic part reads

$$
\begin{aligned}
& \int \prod_{c=1}^{N_{c}} d b_{c} d \bar{b}_{c} \widetilde{d b}_{c} d \overline{\widetilde{b}}_{c} \delta\left(\bar{b}_{c} \overline{\widetilde{b}}_{c}+\widetilde{b}_{c} b_{c}\right) \delta\left(i \bar{b}_{c} \overline{\widetilde{b}}_{c}-i \widetilde{b}_{c} b_{c}\right) \delta\left(\bar{b}_{c} b_{c}-\widetilde{b}_{c} \overline{\widetilde{b}}_{c}\right) \\
= & \int \prod_{c=1}^{N_{c}} d b_{c} d \bar{b}_{c} \widetilde{d b}_{c} d \overline{\widetilde{b}}_{c} \int d k_{1} \exp \left(i k_{1}\left(\bar{b}_{c} \overline{\widetilde{b}}_{c}+\widetilde{b}_{c} b_{c}\right)\right) \\
& \times \int d k_{2} \exp \left(-k_{2}\left(\bar{b}_{c} \overline{\widetilde{b}}_{c}-\widetilde{b}_{c} b_{c}\right)\right) \int d k_{3} \exp \left(i k_{3}\left(\bar{b}_{c} b_{c}-\widetilde{b}_{c} \overline{\widetilde{b}}_{c}\right)\right) .
\end{aligned}
$$

Finally, we combine (3.46), 3.47) and the bosonic fields from the fermionic integration (3.45) into the bosonic integral

$$
\int d^{3} k \int \prod_{c=1}^{N_{c}} d b_{c} \overline{\widetilde{d}}_{c} d \bar{b}_{c} \widetilde{d b}_{c}\left(b_{p} \bar{b}_{q}+\overline{\widetilde{b}}_{p} \widetilde{b}_{q}\right) \exp \left(-\left[\begin{array}{c}
b \\
\overline{\widetilde{b}}
\end{array}\right]^{T}\left[\begin{array}{ll}
M_{(1)} & M_{(2)} \\
M_{(3)} & M_{(4)}
\end{array}\right]\left[\begin{array}{c}
\bar{b} \\
\widetilde{b}
\end{array}\right]\right),
$$

where the $b$ 's are vectors with the $N_{c}$ entries $b_{c}$ and the $M$ 's are $N_{c} \times N_{c}$ matrices of the following form:

$$
\begin{array}{ll}
M_{(1)}=-\Phi \widetilde{\Phi}+\epsilon \mathbb{1}-i k_{3} \mathbb{1}, & M_{(2)}=-i k_{1} \mathbb{1}-k_{2} \mathbb{1}, \\
M_{(3)}=-i k_{1} \mathbb{1}+k_{2} \mathbb{1}, & M_{(4)}=-\Phi \widetilde{\Phi}+\epsilon \mathbb{1}+i k_{3} \mathbb{1} .
\end{array}
$$

Here we have introduced an infinitesimal parameter $\epsilon>0$ in order to regularize the complex Gaussian integrals (3.48). At the end of the computation we will take the $\epsilon \rightarrow 0$ limit.

Note that bosonic fields in front of the exponential can be written as

$$
b_{p} \bar{b}_{q}+\overline{\widetilde{b}}_{p} \widetilde{b}_{q}=-\frac{\partial}{\partial M_{(1) p, q}}-\frac{\partial}{\partial M_{(4) p, q}},
$$

where for instance the first derivative is with respect to the $(p, q)$ 'th element of the matrix $M_{(1)}$.

Then we can perform the Gaussian integrals in 3.48 to obtain

$$
\begin{aligned}
& \int d^{3} k\left(-\frac{\partial}{\partial M_{(1) p, q}}-\frac{\partial}{\partial M_{(4) p, q}}\right) \operatorname{det}\left(\left[\begin{array}{ll}
M_{(1)} & M_{(2)} \\
M_{(3)} & M_{(4)}
\end{array}\right]\right)^{-1} \\
= & \int d^{3} k\left(\left(M_{(1)}^{-1}\right)_{q, p}+\left(M_{(4)}^{-1}\right)_{q, p}\right) \operatorname{det}\left(\left[\begin{array}{cc}
M_{(1)} & M_{(2)} \\
M_{(3)} & M_{(4)}
\end{array}\right]\right)^{-1} \\
= & -2 \int d^{3} k \sum_{r=1}^{N_{c}} \frac{(\Phi \widetilde{\Phi}-\epsilon \mathbb{1})_{q, r}\left((\Phi \widetilde{\Phi}-\epsilon \mathbb{1})^{2}+k^{2} \mathbb{1}\right)_{r, p}^{-1}}{\operatorname{det}\left[(\Phi \widetilde{\Phi}-\epsilon \mathbb{1})^{2}+k^{2} \mathbb{1}\right]}
\end{aligned}
$$


From (3.51) to 3.52 we used formula (D.1) from appendix D. From 3.52 to 3.53 we used (D.2) and (D.3). Note that $M_{(1)}^{-1}$ stands for the upper-left block of the block-matrix $M^{-1}$, i.e. it is not the inverse of $M_{(1)}$.

At this point let us summarize the results of the fermionic and bosonic zero mode integration so far. Combining equations (3.45) and (3.53) and going to spherical coordinates we find the expression

$$
\begin{aligned}
I_{\mathrm{bos}}(\Phi, \widetilde{\Phi})= & \int d^{4} x d^{2} \theta \int_{0}^{\infty} d k k^{2} \\
& \times \sum_{p, q, r=1}^{N_{c}}(-1)^{p+q} \frac{\operatorname{det}\left[\left.\Phi \widetilde{\Phi}\right|_{q, p}\right](\Phi \widetilde{\Phi}-\epsilon \mathbb{1})_{q, r}\left((\Phi \widetilde{\Phi}-\epsilon \mathbb{1})^{2}+k^{2} \mathbb{1}\right)_{r, p}^{-1}}{\operatorname{det}\left[(\Phi \widetilde{\Phi}-\epsilon \mathbb{1})^{2}+k^{2} \mathbb{1}\right]}
\end{aligned}
$$

Note that so far still the combination $\Phi \widetilde{\Phi}$ occurs, whereas eventually we have to get the meson matrix $\widetilde{\Phi} \Phi$. In (3.54) there is a part which can be simplified using equations (D.4) and (D.5) from appendix $D$ :

$$
\sum_{q=1}^{N_{c}}(-1)^{p+q} \operatorname{det}\left[\left.\Phi \widetilde{\Phi}\right|_{q, p}\right] \Phi \widetilde{\Phi}_{q, r}=\operatorname{det}[\Phi \widetilde{\Phi}] \delta_{p, r}=0
$$

The remaining part can be rewritten using equations (D.6) and (D.7) from appendix D

$$
\begin{aligned}
& \int_{0}^{\infty} d k k^{2} \sum_{p, q=1}^{N_{c}}(-1)^{2 p+2 q} \frac{\operatorname{det}\left[\left.\Phi \widetilde{\Phi}\right|_{q, p}\right] \operatorname{det}\left[\left.\left((\widetilde{\Phi}-\epsilon \mathbb{1})^{2}+k^{2} \mathbb{1}\right)\right|_{p, q}\right]}{\operatorname{det}\left[(\Phi \widetilde{\Phi}-\epsilon \mathbb{1})^{2}+k^{2} \mathbb{1}\right]^{2}} \\
= & \epsilon \int_{0}^{\infty} d k k^{2} \frac{\sum_{p=1}^{N_{c}} \operatorname{det}\left[\left.\left(\Phi \widetilde{\Phi}\left((\Phi \widetilde{\Phi}-\epsilon \mathbb{1})^{2}+k^{2} \mathbb{1}\right)\right)\right|_{p, p}\right]}{\operatorname{det}\left[(\Phi \widetilde{\Phi}-\epsilon \mathbb{1})^{2}+k^{2} \mathbb{1}\right]^{2}} .
\end{aligned}
$$

This expression can be simplified using the formulas for the determinant and characteristic polynomial of appendix D

$$
\begin{aligned}
& =\epsilon \int_{0}^{\infty} d k k^{2} \frac{\operatorname{det}\left[\widetilde{\Phi} \Phi\left((\widetilde{\Phi} \Phi-\epsilon \mathbb{1})^{2}+k^{2} \mathbb{1}\right)\right]}{(i k-\epsilon)^{2}(i k+\epsilon)^{2} \operatorname{det}[\widetilde{\Phi} \Phi-\epsilon \mathbb{1}+i k \mathbb{1}]^{2} \operatorname{det}[\widetilde{\Phi} \Phi-\epsilon \mathbb{1}-i k \mathbb{1}]^{2}} \\
& =\epsilon \int_{0}^{\infty} d k k^{2} \frac{\operatorname{det}[\widetilde{\Phi} \Phi]}{(i k-\epsilon)^{2}(i k+\epsilon)^{2} \operatorname{det}[\widetilde{\Phi} \Phi-\epsilon \mathbb{1}+i k \mathbb{1}] \operatorname{det}[\widetilde{\Phi} \Phi-\epsilon \mathbb{1}-i k \mathbb{1}]}
\end{aligned}
$$


Finally, let us denote the eigenvalues of the matrix $\widetilde{\Phi} \Phi$ as $\sigma_{j}$ with $j=1, \ldots, N_{c}-1$. Then we obtain for equation (3.54) the following expression

$$
\begin{aligned}
I_{\mathrm{bos}}(\Phi, \widetilde{\Phi})=\int d^{4} x d^{2} \theta \operatorname{det}[\widetilde{\Phi} \Phi] \\
\quad \times \epsilon \int_{0}^{\infty} d k \frac{k^{2}}{(i k-\epsilon)^{2}(i k+\epsilon)^{2} \prod_{j=1}^{N_{c}-1}\left(\sigma_{j}-\epsilon+i k\right)\left(\sigma_{j}-\epsilon-i k\right)} .
\end{aligned}
$$

We denote the integrand in the last line as $f(k)$. Due to the symmetry $k \rightarrow-k$ of the integral and the vanishing of the integrand at infinity, we can rewrite (3.58) as a contour integral

$$
I_{\mathrm{bos}}(\Phi, \widetilde{\Phi})=\int d^{4} x d^{2} \theta \operatorname{det}[\widetilde{\Phi} \Phi] \frac{\epsilon}{2 \pi i} \oint f(k)
$$

closing the circuit in the upper half-plane, for instance.

The contour integral can be evaluated by virtue of the residue theorem:

$$
\frac{\epsilon}{2 \pi i} \oint f(k)=\epsilon \operatorname{Res}[f(k), k=i \epsilon]+\epsilon \sum_{j=1}^{N_{c}-1} \operatorname{Res}\left[f(k), k=i\left(\sigma_{j}-\epsilon\right)\right] .
$$

Generically, all eigenvalues of the matrix $\widetilde{\Phi} \Phi$ are nonzero and we find for the first term

$$
\epsilon \operatorname{Res}[f(k), k=i \epsilon]=-\frac{i}{4} \frac{1}{\prod_{j=1}^{N_{c}-1}\left(\sigma_{j}-2 \epsilon\right) \sigma_{j}}\left(1+\sum_{j=1}^{N_{c}-1} \frac{2 \epsilon^{2}}{\left(\sigma_{j}-2 \epsilon\right) \sigma_{j}}\right) .
$$

For the calculation of all the other residues in 3.60 we can assume that $\left|\sigma_{j}\right|>\epsilon$. Therefore, the limit $\epsilon \rightarrow 0$ can be taken before evaluating the residue and so all these terms vanish.

Taking the limit $\epsilon \rightarrow 0$ in expression (3.61), the result simplifies drastically and we obtain

$$
\lim _{\epsilon \rightarrow 0} \operatorname{Res}[f(k), k=i \epsilon]=-\frac{i}{4} \frac{1}{\prod_{j=1}^{N_{c}-1} \sigma_{j}^{2}}=-\frac{i}{4} \frac{1}{\operatorname{det}[\widetilde{\Phi} \Phi]^{2}} .
$$

Using this result for the contour integration, we arrive at our final result for the bosonic zero mode integration:

$$
I_{\mathrm{bos}}(\Phi, \widetilde{\Phi})=\int d^{4} x d^{2} \theta \frac{1}{\operatorname{det}[\widetilde{\Phi} \Phi]}
$$

The last step to obtain the E2-instanton generated superpotential $(3.3)$ is to include the contribution of $\exp \left(\langle 1\rangle^{\text {disk }}\right)$

$$
S_{W}=\frac{2 \pi^{2}}{\ell_{s}^{3}} \int d^{4} x d^{2} \theta \frac{1}{\operatorname{det}[\widetilde{\Phi} \Phi]} \exp \left(-\frac{8 \pi^{2}}{g_{c}^{2}\left(M_{s}\right)}\right)
$$


with $M_{s}=\left(\alpha^{\prime}\right)^{-1 / 2}$.

Transforming to canonically normalized matter fields, $\Phi=\left(2 \pi \alpha^{\prime}\right)^{1 / 2} \Phi_{\text {phys }}$, we get

$$
S_{W}=N \int d^{4} x d^{2} \theta \frac{M_{s}^{2 N_{c}+1}}{\operatorname{det}\left[\widetilde{\Phi}_{\text {phys }} \Phi_{\text {phys }}\right]} \exp \left(-\frac{8 \pi^{2}}{g_{c}^{2}\left(M_{s}\right)}\right)
$$

where we have collected all numerical factors appearing during the computation into the constant $N$.

Using (3.39) for the dynamically generated scale $\Lambda$, while absorbing the numerical factor into $\Lambda$, gives precisely the action for the $\operatorname{ADS}$ superpotential

$$
S_{W}=\int d^{4} x d^{2} \theta \frac{\Lambda^{2 N_{c}+1}}{\operatorname{det}\left[M_{f f^{\prime}}\right]} .
$$

Therefore, we have derived the ADS superpotential in the field theory limit from a single E2-instanton. As mentioned, in 'full string theory' there will be numerous corrections to this simple expression. All the massive string states will appear in the one-loop determinant and there will be an infinite series of world-sheet instanton corrections to the disk amplitudes. Moreover, multiple matter field insertions along the boundary of the disk are possible and give higher $\alpha^{\prime}$ corrections to the ADS superpotential. Note that these higher order terms could break some of the global symmetries, such as R-symmetry, present in the field theory limit.

For this reason, a warning is in order at this point: Whenever one simply extends, for instance, string flux superpotentials by non-perturbative superpotentials from pure field theory, like the ADS superpotential, one has to ensure that one always remains in the regime of validity of the field theory limit of string theory, i.e. at large transversal radii and 'small' vevs for the matter fields, i.e. $\langle\Phi\rangle \ll M_{s}$.

\subsection{Relation to Field Theory}

At several point in the previous sections we referred to the review [70] to point out that our effective terms, in the field theory limit, in formula (3.3) agreed with the calculation of the ADS superpotential in the general instanton calculus context.

There, the ADHM constraints relating the instanton zero modes arise from an explicit construction. In our stringy derivation of the ADS superpotential, the ADHM constraints arise from the dynamics, so to speak, of the E2-instanton and are not the result of some mathematical construction. Moreover, the effective terms $\mathcal{L}_{\text {bos, ferm }}$ arise from CFT disk diagrams in the formula (3.3) for calculating the E2-instanton contribution to the superpotential. It should be noted that the stringy approach in principle allows to calculate 
corrections beyond the field theory limit. Therefore, the question arises, why do they agree in the field theory limit?

Put this way, the blunt answer is: because they have to. We remarked in the introduction to this chapter that one purpose of the developments presented here was to verify that the stringy instanton calculus of [34] gives the correct result in a known case.

However, from a perspective not tied to the stringy instanton calculus, there is also a general reason why Ep-instantons reproduce field theory instanton effects. In fact, Dbranes provide a 'physical' understanding of the ADHM construction, with the general lesson that an Ep-instanton 'inside' a $\mathrm{D}(p+4)$-brane gives, in the field theory limit, the same effect as a field theory instanton [149, 71, 72], see also [69, 70].

One should, however, bear in mind that Ep-instantons trivially are not the same as field theory instantons. The former are D-branes with Euclidean world-volume which show up as Euclidean boundaries in CFT amplitudes, whereas the latter are finite action solutions of the Euclidean field equations. A bona fide field theory is defined by its Lagrangian from which one deduces the consequences of the theory, in particular, quantum corrections due to instantons. However, if the field theory is merely the low energy approximation of some string theory, there can in principle appear terms in the effective Lagrangian which do not have an interpretation as the effect of field theory instanton: $5^{5}$ but still arise from E $p$ instantons, albeit ones not inside a D-brane. Such effects have at times been referred to as 'exotic instantons' (see, for instance, [15]). We would like to stress that from the viewpoint of string theory we are not dealing with anything exotic at all. 'Exotic' instanton effects arise from Ep-instantons just as the ADS superpotential arises from an E2-instanton and there definitely is more to string theory than just the low energy effective field theory coming out of it. As a consequence, the effects of Ep-instantons need not be confined to the low energy effective field theory.

There is another way in which field theory instantons differ from spacetime instantons (Epinstantons) in string theory which also has to do with the definitions of the theories. For field theory it should be stressed that we have, in principle, a non-perturbative definition, namely the Euclidean path integral. For string theory this is not the case and some amount of guesswork is required in extending the perturbative scheme. One has to make do with the idea of some (fictitious) grand string path integral and works by analogy to field theory (cf. the remarks in [8]) 6 The basic arguments that $e^{-C / g_{s}}$ weighted contributions to $g_{s}$ dependent amplitudes in the type II theories are then related to E $p$-instantons go back to Polchinski [120].

It is tempting to speculate whether any future metamorphosis of string theory will allow one to deduce the spacetime instanton contribution to observables. However, in absence of any such formulation, we might as well ask whether it is absolutely necessary to include

\footnotetext{
${ }^{5}$ This appears to have been questioned, at least for some cases, in 102 .

${ }^{6}$ Note that the situation is completely different for world-sheet instantons [66, 67. They are conceptually much simpler, as they are instantons of a two-dimensional field theory (the string sigma-model).
} 
them. In our opinion the answer is affirmative. There are at least two ways to argue. Following Polchinski, we could say that spacetime instanton effects are necessary for the consistency of the perturbative expansion of amplitudes. This is an 'internal' argument hinging on the self-consistency of string theory. But in light of the developments of this chapter, we could also argue that spacetime instantons, equivalently Euclidean boundaries in CFT amplitudes, are necessary to reproduce known field theory instanton effects. 


\section{Chapter 4}

\section{One-loop Corrections to Gauge Kinetic Functions ${ }^{\mathbb{1}}$}

\footnotetext{
${ }^{1}$ This chapter is based, sometimes verbatim, on the papers [10, 9] together with R. Blumenhagen, D. Lüst, and M. Schmidt-Sommerfeld.
} 


\subsection{Introduction}

At low energies, intersecting D6-branes brane models are approximated by a super YangMills theory coupled to four-dimensional supergravity, the precise form depending on the particular construction.

Much of the effort in model building already goes into getting the gauge groups and chiral spectrum right. However, there is more to the (Wilsonian) low energy effective action than just these data. Of particular interest are also the gauge kinetic functions $f_{a}$, superpotential $W$, and the matter Kähler potential $\mathcal{K}$. Thus, in order to build concrete models one needs to have a handle on computing these too.

Of these quantities, the first two are holomorphic functions. In addition, there are general non-renormalization theorems: The gauge kinetic functions can receive one-loop and nonperturbative corrections, while the superpotential is at most corrected non-perturbatively. The matter Kähler potential, however, is not protected from corrections.

In this chapter we analyze one-loop corrections to the gauge kinetic functions. First, namely in section 4.2, we give a non-renormalization theorem constraining the dependence of the gauge kinetic functions on the complex structure and Kähler moduli (for completeness we also remark on the superpotential). In section 4.3, we turn to the gauge threshold corrections for intersecting D6-brane models on the $\mathbb{Z}_{2} \times \mathbb{Z}_{2}$ toroidal orbifold ${ }^{2}$ [77, 56, 55]. After that, in section 4.4, we investigate the appearance of non-holomorphic terms in the gauge threshold corrections and how it is nevertheless possible to extract the one-loop corrections to the gauge kinetic functions from them. Finally, we illustrate this on the example of the $\mathbb{Z}_{2} \times \mathbb{Z}_{2}$ toroidal orbifold.

\subsection{Non-renormalization Theorems}

In this section we investigate the structure of perturbative and non-perturbative corrections to the gauge kinetic functions and superpotential for intersecting D6-brane models. We are only concerned with D6-brane constructions preserving $\mathcal{N}=1$ supersymmetry in four-dimensions. Recall that in such constructions the stacks of D6-branes wrap special Lagrangian three-cycles $\Pi_{a}$ in the orientifolded Calabi-Yau or toroidal orbifold $X$ on which we compactify.

On $X$ we select a symplectic basis $\left(A_{I}, B^{I}\right), I=0,1, \ldots, h_{2,1}$ of homological three-cycles with the topological intersection numbers

$$
A_{I} \circ B^{J}=\delta_{I}^{J}
$$

\footnotetext{
${ }^{2}$ There is some ambiguity in speaking of $\mathbb{Z}_{2} \times \mathbb{Z}_{2}$ toroidal orbifolds. We will refer to the one with $\left(h_{21}, h_{11}\right)=(3,51)$ as $\mathbb{Z}_{2} \times \mathbb{Z}_{2}$ and to the one with $\left(h_{21}, h_{11}\right)=(51,3)$ as $\mathbb{Z}_{2} \times \mathbb{Z}_{2}^{\prime}$.
} 
Further, we assume that the $A_{I}$ cycles are invariant under the orientifold projection and that the $B^{J}$ cycles are projected out.

The complexified complex structure moduli $U_{I}^{c}$ on such an orientifold are defined as

$$
U_{I}^{c}=\frac{1}{(2 \pi) \ell_{s}^{3}}\left[e^{-\phi_{4}} \int_{A_{I}} \operatorname{Re}\left(\widehat{\Omega}_{3}\right)-i \int_{A_{I}} C_{3}\right],
$$

where $\widehat{\Omega}_{3}$ denotes the (unique) normalized holomorphic three-form on $X, C_{3}$ is the type IIA $\mathrm{R}-\mathrm{R}$ three-form, and the four-dimensional dilaton $\phi_{4}$ is defined from the ten-dimensional one $\phi_{10}$ by $\phi_{4}=\phi_{10}-\frac{1}{2} \log \left(V_{X} / \ell_{s}^{6}\right)$. Since the imaginary parts of the $U_{I}^{c}$ are axionic fields, they enjoy a Peccei-Quinn shift symmetry $U_{I}^{c} \rightarrow U_{I}^{c}+c_{I}$ which is preserved perturbatively but can be broken by E2-instantons.

Expanding the three-cycle $\Pi_{a}$ wrapped by brane stack $a$ in our symplectic basis,

$$
\Pi_{a}=M_{a}^{I} A_{I}+N_{a, I} B^{I},
$$

with $M^{I}, N_{I} \in \mathbb{Z}$, from dimensional reduction of the Dirac-Born-Infeld (DBI) action one can deduce that the gauge kinetic function at string tree-level is [35]

$$
f_{a}^{\mathrm{tree}}=\sum_{I=0}^{h_{2,1}} M_{a}^{I} U_{I}^{c} .
$$

Let $C_{i}$ denote a basis of two-cycles anti-invariant under the orientifold projection, i.e. $C_{i} \in H_{-}^{1,1}$. The complexified Kähler moduli are then defined as

$$
T_{i}^{c}=\frac{1}{\ell_{s}^{2}}\left(\int_{C_{i}} J_{2}-i \int_{C_{i}} B_{2}\right),
$$

where $B_{2}$ denotes the type IIA NS-NS two-form. From this we see that also the complexified Kähler moduli enjoy a Peccei-Quinn shift symmetry, possibly broken by world-sheet instantons. Note that the chiral fields $T_{i}^{c}$ organize the $\alpha^{\prime}$ perturbation theory and do not contain the dilaton. Hence, the perturbative expansion in $g_{s}$ is entirely defined in terms of powers of the $U_{I}^{c}$. In the following, to shorten the notation we denote the real parts of $U_{I}^{c}$ and $T_{i}^{c}$ by $U_{I}$, respectively $T_{i}$.

The superpotential $W$ and the gauge kinetic functions $f_{a}$ in the four-dimensional effective supergravity action are holomorphic quantities. In the usual way, employing holomorphy and the Peccei-Quinn symmetries above, one arrives at the following two nonrenormalization theorems, giving restrictions on the dependence on the complex structure and Kähler moduli:

- The superpotential can only have the following dependence on the $U_{I}^{c}$ and $T_{i}^{c}$ :

$$
W=W^{\text {tree }}+W^{\text {np }}\left(e^{-U_{I}^{c}}, e^{-T_{i}^{c}}\right) .
$$

Thus, beyond tree-level there can only be non-perturbative contributions from worldsheet $\left(\sim e^{-1 / \alpha^{\prime}}\right)$ and E2-instantons $\left(\sim e^{-1 / g_{s}}\right)$. 
- Similarly, the holomorphic gauge kinetic function must look like

$$
f_{a}=\sum_{I} M_{a}^{I} U_{I}^{c}+f_{a}^{1-\mathrm{loop}}\left(e^{-T_{i}^{c}}\right)+f_{a}^{\mathrm{np}}\left(e^{-U_{I}^{c}}, e^{-T_{i}^{c}}\right) .
$$

In particular, its one-loop correction must not depend on the complex structure moduli, a result which will be important in deducing $f_{a}^{1-l o o p}$ from gauge threshold corrections.

\subsection{Threshold Corrections for $T^{6} / \mathbb{Z}_{2} \times \mathbb{Z}_{2}$ Models}

In intersecting D6-brane models a gauge theory is induced on each stack of branes. From the four-dimensional perspective this means that there will be a gauge group $\prod_{a} G_{a}$ consisting of the product of all the factors coming from the different stacks. With each group factor $G_{a}$ goes a separate gauge coupling. In the tree-level approximation of the topological expansion of the gauge coupling $g_{a}$ on brane stack $a$, we have the relation at the string scale

$$
\frac{1}{g_{a, \text { tree }}^{2}} \sim \operatorname{volume}\left(\Pi_{a}\right)
$$

with $\Pi_{a}$ the cycle which stack $a$ wraps in the internal manifold. We see that the different couplings can very well have different magnitude which, for a high string scale, would be at variance with the kind of gauge coupling unification present in the MSSM.

The formula (4.8) we have written down for the gauge couplings can receive one-loop (threshold), higher loop, and non-perturbative corrections. The one-loop corrections can sometimes restore a unification of couplings [36] which is one reason to be interested in them. However, here our main interest in them rests with the fact, to be explained in section 4.4, that they can be used to infer the one-loop corrections to the gauge kinetic functions.

In this section we redo [10] the regularization of the gauge threshold corrections in [110] for intersecting D6-branes on the $\mathbb{Z}_{2} \times \mathbb{Z}_{2}$ toroidal orbifold. In fact, the raw amplitudes from which one obtains the threshold corrections exhibit ultraviolet and infrared divergences. The ultraviolet divergences are taken care of for supersymmetric brane configurations by the tadpole cancellation condition. The infrared divergences are due to massless open string modes. For heterotic models there appears to be a general method for dealing with these divergences [100] which couples the theory to a (four-dimensional) curved background whereby providing a 'universal' regularization scheme. An adaption of this method would presumably also work in the case at hand. However, here we present two different regularization schemes for $\mathcal{N}=1$ open string sectors which a posteriori turn out to be equivalent. In the first approach, we compute in tree channel where the ultraviolet tadpoles are easily extracted. The infrared divergences are then removed by dimensional regularization. In the second approach, we work in the loop channel. In this channel the 
infrared divergences are manifest and the remaining ultraviolet divergences are cancelled by zeta-function regularization.

Carrying out the program thus outlined for $\mathcal{N}=1$ sectors, we find that we get something slightly different than in [110]. The dimensional regularization in the tree channel is under good control, convincing us that our results are indeed correct. For completeness, we also give a particular regularization for $\mathcal{N}=2$ sectors, whose results are in agreement with [110].

\subsubsection{Background and Calculations}

The one-loop corrections to the gauge coupling constants in intersecting D6-brane models can be computed by means of the background field method [19, 11, 110] which essentially amounts to computing the partition function in the presence of a magnetic field in fourdimensional spacetime.

The gauge coupling constants of the various gauge group factors $G_{a}$, up to one loop, have the form

$$
\frac{8 \pi^{2}}{g_{a}^{2}(\mu)}=\frac{8 \pi^{2}}{g_{a, \text { string }}^{2}}+\frac{b_{a}}{2} \log \left(\frac{M_{s}^{2}}{\mu^{2}}\right)+\frac{\Delta_{a}}{2},
$$

where $b_{a}$ is the beta function coefficient. The first term on the right is the gauge coupling constant at the string scale which contains the tree-level gauge coupling (4.8) as well as 'universal' contributions at one-loop. The second term gives the familiar one-loop running of the coupling constants, and the third term, $\Delta_{a}$, denotes the one-loop string threshold corrections originating from integrating out massive string excitations.

The one-loop corrections to the tree-level string scale gauge coupling (4.8) are universal in the sense that they originate from a redefinition of the dilaton and complex structure moduli at one-loop. This redefinition is brane-stack and therefore gauge group independent. However, as the gauge coupling typically already is gauge group dependent at tree level, the complete term $g_{a \text {,string }}^{2}$ effectively is gauge group dependent. For more on the universal corrections see section 4.4.1.

The last two terms in 4.9 ) are encoded in the partition function mentioned above and can therefore be determined by calculating all annulus and Möbius diagrams with at least one boundary on the brane where the gauge group factor $G_{a}$ is localized.

For the contribution of an annulus diagram to the threshold corrections for relatively supersymmetric intersecting branes $\mathrm{D} 6_{a}$ and $\mathrm{D} 6_{b}$ on a Calabi-Yau or toroidal orbifold, the background field method gives the general expression [110]

$$
T^{A}\left(\mathrm{D} 6_{a}, \mathrm{D} 6_{b}\right)=\int_{0}^{\infty} \frac{d t}{t} \sum_{\alpha, \beta \neq\left(\frac{1}{2}, \frac{1}{2}\right)}(-1)^{2(\alpha+\beta)} \frac{\vartheta^{\prime \prime}\left[\begin{array}{c}
\alpha \\
\beta
\end{array}\right](i t)}{\eta^{3}(i t)} A_{a b}\left[\begin{array}{c}
\alpha \\
\beta
\end{array}\right](i t)
$$


where $A_{a b}$ denotes the annulus partition function in the $(a b)$ open string sector of the internal $\mathcal{N}=2$ superconformal field theory associated with the Calabi-Yau, respectively toroidal orbifold. There is an analogous formula for the Möbius diagram contributions $T^{M}\left(\mathrm{D} 6_{a}, \mathrm{O} 6_{k}\right)$ and the sum of all such conceivable contributions then gives us $b_{a} \log \left(\frac{M_{s}^{2}}{\mu^{2}}\right)+$ $\Delta_{a}$ above.

More precisely, we have the equation

$$
\begin{aligned}
b_{a} \log \left(\frac{M_{s}^{2}}{\mu^{2}}\right)+\Delta_{a}= & \sum_{b} T^{A}\left(\mathrm{D} 6_{a}, \mathrm{D} 6_{b}\right)+\sum_{b^{\prime}} T^{A}\left(\mathrm{D} 6_{a}, \mathrm{D} 6_{b^{\prime}}\right) \\
& +T^{A}\left(\mathrm{D} 6_{a}, \mathrm{D} 6_{a^{\prime}}\right)+T^{M}\left(\mathrm{D} 6_{a}, \mathrm{O} 6\right) .
\end{aligned}
$$

Here, $\mathrm{D} 6_{c^{\prime}}$ denotes the orientifold image of brane $c$.

After these general considerations we will now specialize to the case of the toroidal $\mathbb{Z}_{2} \times \mathbb{Z}_{2}$ orbifold. If we were to construct an intersecting D6-brane model in this background we would first have a number of O6-planes coming from the necessary orientifolding. Furthermore, we would then introduce various stacks $a$ of D6-branes wrapping factorizable supersymmetric three-cycles defined by three pairs of wrapping numbers $\left(m_{a}^{I}, n_{a}^{I}\right), I=1,2,3$. For consistency, the brane intersections should be relatively supersymmetric, giving certain conditions on the intersection angles (see e.g. [108]). We assume the construction to be such that on each stack there lives a $U\left(N_{a}\right)$ gauge theory and we are interested in the one-loop gauge threshold corrections to the gauge couplings of these theories. In line with the remarks above, the one-loop thresholds are given by annulus and Möbius diagrams with one boundary on the $U\left(N_{a}\right)$ brane. For future use, we remark that the intersection angles $\theta$ are (non-holomorphic) functions of the complex structure moduli alone [110].

\section{Regularization for $\mathcal{N}=1$ sectors}

Tree channel calculation. We now come to the actual regularization of amplitudes. We take as our starting point the raw amplitudes found in [110]. For the annulus diagram in an $\mathcal{N}=1$ sector, the expression to be examined is

$$
\begin{aligned}
T^{A}\left(\mathrm{D} 6_{a}, \mathrm{D} 6_{b}\right) & =\frac{i I_{a b} N_{b}}{2 \pi} \int_{0}^{\infty} \frac{d t}{t} \sum_{I=1}^{3} \frac{\vartheta_{1}^{\prime}}{\vartheta_{1}}\left(\frac{i \theta_{a b}^{I} t}{2}, \frac{i t}{2}\right) \\
& =-\frac{I_{a b} N_{b}}{\pi} \int_{0}^{\infty} d l \sum_{I=1}^{3} \frac{\vartheta_{1}^{\prime}}{\vartheta_{1}}\left(-\theta_{a b}^{I}, 2 i l\right),
\end{aligned}
$$

where $I_{a b}$ is the intersection number, $N_{b}$ is the number of branes on stack $b$ and $l=1 / t$. Additionally, $\pi \theta_{a b}^{I}$ is the intersection angle of branes $a$ and $b$ on the $I$ 'th torus. The specialization to an $\mathcal{N}=1$ sector translates to the condition on intersection angles

$$
\sum_{I=1}^{3} \theta_{a b}^{I}=0
$$


which we assume to be fulfilled.

As it stands, (4.12) is divergent. As mentioned above, there are two sources for this divergence: In the $q$-series of the $l$-channel integrand $\sum \frac{\vartheta_{1}^{\prime}}{\vartheta_{1}}\left(-\theta_{a b}^{I}, 2 i l\right)$ there is a constant (i.e. $q^{0}-$ ) term, giving us something proportional to $\int d l$. Even if this is subtracted (or thought of as taken care of by tadpole cancellation) there remains a divergence from the $l \rightarrow 0$ region of integration. The latter is the same as the divergence for $t \rightarrow \infty$ coming from the constant term in the loop channel. It is therefore seen to be a logarithmic divergence which arises from the massless open string states and encodes the one-loop running of the gauge couplings. Thus, it will later on be replaced by $\log \left(\frac{M_{s}^{2}}{\mu^{2}}\right)$.

Since, following [110, we impose the tadpole cancellation condition, we do not worry about the $q^{0}$-divergence, but clearly something needs to be done about the remaining logarithmic divergence. There are at least two ways to proceed. One is to subtract this divergence in the $t$-channel and another is to employ dimensional regularization. If one wants to extract the tadpole divergence manifestly, which is only possible in tree channel, at least the large $l$ (i.e. small $t \propto 1 / l)$ part of the amplitude has to be calculated in tree channel. This means that one can only work in the large $t$ regime of the loop channel or, in other words, that the $t$-integration has to be cut off at a finite lower limit. Since the computation is then difficult to do analytically, we shall carry out the tree channel computation using dimensional regularization $\mathrm{I}^{3}$

It is easy to derive the following formuld for the $l$-channel summands in 4.12 (cf. 4.34) below):

$$
-\frac{1}{\pi} \int_{0}^{\infty} d l \frac{\vartheta_{1}^{\prime}}{\vartheta_{1}}(\theta, 2 i l)=-\cot (\pi \theta) \int_{0}^{\infty} d l+A
$$

where

$$
A=4 i \int_{0}^{\infty} d l \sum_{m, n=1}^{\infty} \exp (-4 \pi l m n) \sinh (2 \pi i \theta m)
$$

When taking the sum over the $\theta$ s, (4.14) gives us (4.12) (up to prefactors), so we might as well regularize (4.14). The first term in (4.14) is the tadpole, which we assume to be canceled, and the second is the infrared divergence which we have to regularize. The way we do this is to put $\int d l \rightarrow \int d l l^{\epsilon}$, where $\epsilon$ is a (small) positive number. In loop channel this amounts to the substitution $\int \frac{d t}{t} \rightarrow \int \frac{d t}{t^{1+\epsilon}} \simeq \frac{1}{\epsilon}$. In analogy to the heterotic string [95], it is therefore justified to later substitute $\log \left(\frac{M_{s}^{2}}{\mu^{2}}\right)$ for $\frac{1}{\epsilon}$.

\footnotetext{
${ }^{3}$ This method of regularization has already been put to work in [110], however with a slightly different result than ours.

${ }^{4}$ Note the seemingly different formula for $\frac{\vartheta_{1}^{\prime}}{\vartheta_{1}}$ as the one in [110]. The latter can be brought into the form given here by performing the sum over $k$ there.
} 


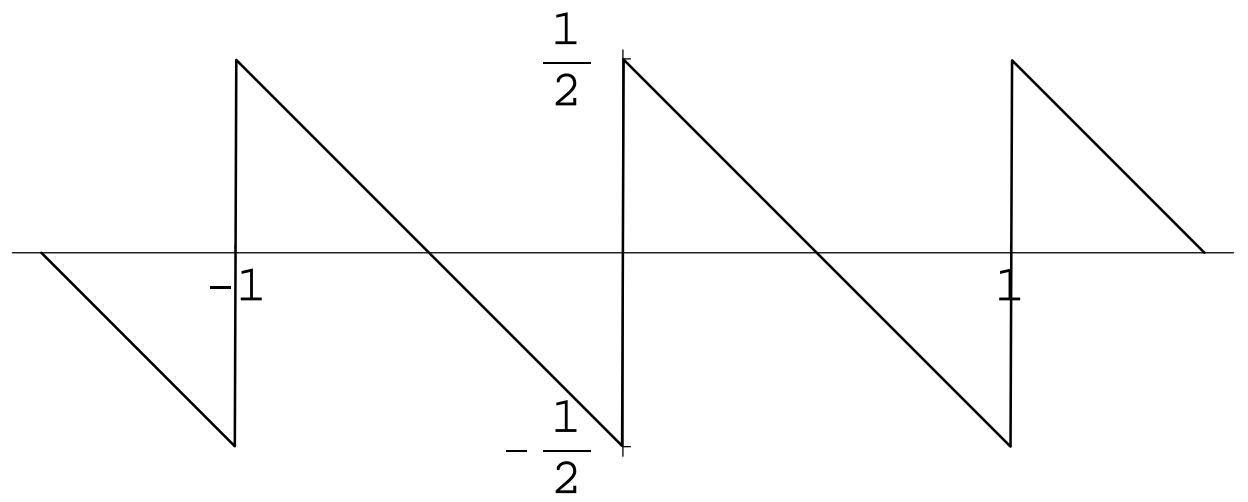

Figure 4.1: Saw-tooth function.

Integrating over $l$ and carrying out the sum over $n$ in (4.15) yields

$$
A=-\frac{1}{\pi} \sum_{m=1}^{\infty} \frac{\sin (2 \pi \theta m)}{m(4 \pi m)^{\epsilon}} \Gamma(1+\epsilon) \zeta(1+\epsilon) .
$$

For $\epsilon \ll 1$, we can expand

$$
A=-\frac{1}{\epsilon}\left(\frac{1}{\pi} \sum_{m=1}^{\infty} \frac{\sin (2 \pi \theta m)}{m}\right)+\frac{1}{\pi} \sum_{m=1}^{\infty} \frac{\log (4 \pi m)}{m} \sin (2 \pi \theta m)+O(\epsilon),
$$

from which the $\epsilon \rightarrow 0^{+}$divergence is nicely read off. The term in parentheses is a standard example of a Fourier series. In the open interval ]0, 1 [ it sums to $1 / 2-\theta$, while for $\theta=0$ it gives zero and elsewhere it sums to formulas given by periodic continuation with period 1. In fact, it is just the saw-tooth function in figure 4.1. Upon performing the sum over $I$ in 4.12 and using 4.13 one finds that the $\frac{1}{\epsilon}$-term is multiplied by a constant, which, taking into account the prefactors in (4.12), is just the contribution of brane stack $b$ to the beta function. Thus, upon substituting $\frac{1}{\epsilon} \rightarrow \log \left(\frac{M_{s}^{2}}{\mu^{2}}\right)$, the correct one-loop running is reproduced. It remains to sum the left-over Fourier series

$$
\frac{1}{\pi} \sum_{m=1}^{\infty} \frac{\log (4 \pi m)}{m} \sin (2 \pi \theta m)
$$

By Dirichlet's test, it converges for all $\theta$ in, say, the open interval ]0,1[. Moreover, it converges trivially to zero for $\theta=0$, and therefore it converges for all $\theta$ by periodicity.

It turns out that, for $0<\theta<1$,

$$
\frac{1}{\pi} \sum_{m=1}^{\infty} \frac{\log (4 \pi m)}{m} \sin (2 \pi \theta m)=\frac{1}{2} \log \left(\frac{\Gamma(\theta)}{\Gamma(1-\theta)}\right)-(\log 2-\gamma)(\theta-1 / 2),
$$

where $\gamma$ is the Euler-Mascheroni constant. 
A heuristic way to arive at the expression on the right hand side of this equation is presented below. Presently, let us show that the relation $(4.19)$ is true. The idea is to interpret the left hand side of (4.19) as the Fourier series of its right hand side (for the theory of Fourier series of functions with infinities like the one at hand see [84]).

The even terms in this Fourier series are all zero for reasons of symmetry 5 , while for the odd terms we have to calculate the sine Fourier coefficients

$$
b_{m}:=2 \int_{0}^{1} d \theta F(\theta) \sin (2 \pi m \theta),
$$

for $m=1,2,3, \ldots$, with $F(\theta):=\frac{1}{2} \log \left(\frac{\Gamma(\theta)}{\Gamma(1-\theta)}\right)-(\log 2-\gamma)(\theta-1 / 2)$.

The only non-trivial integrals arising in this computation are those from the first term in $F(\theta)$

$$
J_{m}:=\int d \theta \log \left(\frac{\Gamma(\theta)}{\Gamma(1-\theta)}\right) \sin (2 \pi m \theta),
$$

while the second term contributes $(\log 2-\gamma) /(m \pi)$.

In order to proceed, we employ the expansion

$$
\log (\Gamma(\theta))=-\gamma \theta-\log (\theta)+\sum_{k=1}^{\infty}\left[\frac{\theta}{k}-\log \left(1+\frac{\theta}{k}\right)\right] .
$$

With this, the integrals $J_{m}$ are easily calculated:

$$
\begin{aligned}
J_{m}= & -2 \gamma \int_{0}^{1} d \theta \theta \sin (2 \pi m \theta)+\int_{0}^{1} d \theta \sin (2 \pi m \theta) \log \left(\frac{1-\theta}{\theta}\right)+ \\
& +\sum_{k=1}^{\infty} \int_{0}^{1} d \theta \theta \sin (2 \pi m \theta)\left(\frac{2 \theta+1}{k}+\log \left(\frac{k+1-\theta}{k+\theta}\right)\right) \\
= & \frac{\gamma}{m \pi}+\frac{\gamma-\operatorname{Ci}[2 \pi m]+\log (2 \pi m)}{m \pi}+ \\
& +\frac{1}{m \pi}\left[\sum_{k=1}^{\infty}(\operatorname{Ci}[2 k m \pi]-\operatorname{Ci}[2(k+1) m \pi])-\sum_{k=1}^{\infty}\left(\frac{1}{k}-\log \left(1+\frac{1}{k}\right)\right)\right],
\end{aligned}
$$

where $\mathrm{Ci}(x)=-\int_{x}^{\infty} \frac{\cos t}{t} d t$ is the cosine integral. Almost all terms in the sums cancel, so that one eventually obtains the simple expression

$$
\begin{aligned}
J_{m} & =\frac{1}{m \pi}\left[2 \gamma+\log (2 \pi m)-\lim _{N \rightarrow \infty} \operatorname{Ci}[2(N+1) m \pi]-(\log \Gamma(1)+\gamma+\log 1)\right] \\
& =\frac{\gamma+\log (2 \pi m)}{m \pi}
\end{aligned}
$$

\footnotetext{
${ }^{5}$ The rhs of 4.19 is odd under reflection of $\theta$ in $1 / 2$, i.e. under $\theta \rightarrow 1-\theta$.
} 
Therefore, collecting terms, we find

$$
b_{m}=\frac{\gamma+\log (2 \pi m)}{m \pi}+\frac{\log 2-\gamma}{m \pi}=\frac{\log (4 \pi m)}{\pi m},
$$

as was to be shown.

The upshot of this discussion is that we have regularized, for $0<\theta<1$, (suppressing the tadpole and $\log \left(M_{s}^{2} / \mu^{2}\right)$ terms $)$

$$
-\frac{1}{\pi} \int_{0}^{\infty} d l \frac{\vartheta_{1}^{\prime}}{\vartheta_{1}}(\theta, 2 i l) \rightarrow \frac{1}{2} \log \left(\frac{\Gamma(\theta)}{\Gamma(1-\theta)}\right)-(\log 2-\gamma)(\theta-1 / 2) .
$$

Now, in view of the angle condition (4.13), it is inevitable that some $\theta$ s are going to be negative, so that we also have to consider the case $-1<\theta<0$. But this is easily reduced to the already derived formulas, with the result (put $-\theta=: \tilde{\theta}>0$ and apply (4.19)):

$$
-\frac{1}{\pi} \int_{0}^{\infty} d l \frac{\vartheta_{1}^{\prime}}{\vartheta_{1}}(\theta, 2 i l) \rightarrow \frac{1}{2} \log \left(\frac{\Gamma(1+\theta)}{\Gamma(-\theta)}\right)-(\log 2-\gamma)(\theta+1 / 2)
$$

for $-1<\theta<0$.

Now we are finally in a position to write down the complete regularized annulus amplitude (4.12). The result is (still suppressing the tadpole):

$$
\begin{aligned}
T^{A}\left(\mathrm{D} 6_{a}, \mathrm{D} 6_{b}\right)=\frac{I_{a b} N_{b}}{2} & {\left[\log \left(\frac{M_{s}^{2}}{\mu^{2}}\right) \sum_{I=1}^{3} \operatorname{sign}\left(\theta_{a b}^{I}\right)-\right.} \\
& \left.-\log \prod_{I=1}^{3}\left(\frac{\Gamma\left(\left|\theta_{a b}^{I}\right|\right)}{\Gamma\left(1-\left|\theta_{a b}^{I}\right|\right)}\right)^{\operatorname{sign}\left(\theta_{a b}^{I}\right)}-\sum_{I=1}^{3} \operatorname{sign}\left(\theta_{a b}^{I}\right)(\log 2-\gamma)\right],
\end{aligned}
$$

where we have taken into account the angle condition 4.13).

The calculation of the Möbius diagrams,

$$
\begin{aligned}
T^{M}\left(\mathrm{D} 6_{a}, \mathrm{O} 6_{k}\right) & = \pm \frac{i 4 I_{a ; \mathrm{O} 6_{k}}}{\pi} \int_{0}^{\infty} \frac{d t}{t} \sum_{I=1}^{3} \frac{\vartheta_{1}^{\prime}}{\vartheta_{1}}\left(i \theta_{a ; \mathrm{O} 6_{k}}^{I} t, \frac{i t}{2}+\frac{1}{2}\right) \\
& = \pm \frac{4 I_{a ; \mathrm{O} 6_{k}}}{\pi} \int_{0}^{\infty} d l \sum_{I=1}^{3} \frac{\vartheta_{1}^{\prime}}{\vartheta_{1}}\left(\theta_{a ; \mathrm{O} 6_{k}}^{I}, 2 i l-\frac{1}{2}\right)
\end{aligned}
$$

proceeds in a rather similar fashion. Here, $I_{a ;{ } 6_{k}}$ is the intersection number of the D-brane and the orientifold plane $k, \theta_{a ; 6_{k}}^{I}$ is the intersection angle of brane $a$ and the orientifold plane $k$ on the $I^{\prime}$ th torus and $l=1 /(4 t)$. The additional summand $-\frac{1}{2}$ in the second 
argument of the theta functions in 4.29) leads to an additional $(-1)^{m n}=\frac{1}{2}\left(1+(-1)^{n}+\right.$ $\left.(-1)^{m}-(-1)^{m+n}\right)$ in the expression corresponding to (4.15). Eventually, one finds

$$
\begin{aligned}
& T^{M}\left(\mathrm{D} 6_{a}, \mathrm{O}_{k}\right)= \pm I_{a ;} \mathrm{O}_{k} \sum_{I=1}^{3} {\left[4 \theta_{a ; \mathrm{O} 6_{k}}^{I}\left(-\log \left(\frac{M_{s}^{2}}{\mu^{2}}\right)+2 \log 2-\gamma\right)\right.} \\
&\left.+\log \left(\frac{M_{s}^{2}}{\mu^{2}}\right) f\left(\theta_{a ; \mathrm{O}_{k}}^{I}\right)+g\left(\theta_{a ; \mathrm{O} 6_{k}}^{I}\right)\right]
\end{aligned}
$$

where the first term vanishes after imposing the supersymmetry condition 4.13,

$$
f(\theta)=\left\{\begin{array}{cl}
\operatorname{sign}(\theta) & \text { for }-\frac{1}{2}<\theta<\frac{1}{2} \\
-3 & \text { for }-1<\theta<-\frac{1}{2} \\
3 & \text { for } \quad \frac{1}{2}<\theta<1
\end{array}\right.
$$

and

$$
g(\theta)=\left\{\begin{array}{cl}
(\gamma-3 \log 2) \operatorname{sign}(\theta)-\operatorname{sign}(\theta) \log \left(\frac{\Gamma(2|\theta|)}{\Gamma(1-2|\theta|)}\right) & \text { for }-\frac{1}{2}<\theta<\frac{1}{2} \\
-3 \gamma+5 \log 2+\log \left(\frac{\Gamma(-2 \theta-1)}{\Gamma(2+2 \theta)}\right) & \text { for }-1<\theta<-\frac{1}{2} \\
3 \gamma-5 \log 2-\log \left(\frac{\Gamma(2 \theta-1)}{\Gamma(2-2 \theta)}\right) & \text { for } \quad \frac{1}{2}<\theta<1 .
\end{array}\right.
$$

Cases where the intersection angles sum to $\pm 2 n, n \in \mathbb{N}^{*}$, can be treated by periodic continuation of our formulas (cf. 4.17) and 4.19).

Loop channel calculation and zeta-function regularization. The dimensional regularization of amplitudes in the tree channel presented above is solid but there remains the question how to arrive at, e.g., (4.19). Recall that we only stated the result and then, by Fourier analysis proved that it is true. Therefore, as already announced earlier, we now compute the analytic form 4.19 of the $\mathcal{N}=1$ sector annulus threshold corrections using zeta-function regularization in the loop channel. This method of calculation reproduces the threshold corrections up to an additive constant, i.e. in particular the full moduli $(\theta)$ dependence will come out right. For the annulus diagram, this means evaluating

$$
T^{A}\left(\mathrm{D} 6_{a}, \mathrm{D} 6_{b}\right)=\frac{i I_{a b} N_{b}}{2 \pi} \int_{0}^{\infty} \frac{d t}{t} \sum_{I=1}^{3} \frac{\vartheta_{1}^{\prime}}{\vartheta_{1}}\left(\frac{i \theta_{a b}^{I} t}{2}, \frac{i t}{2}\right)
$$

Using the product representation of the theta function and the Taylor expansion $\log (1-$ $z)=-\sum_{n=1}^{\infty} \frac{z^{n}}{n}$ one can derive

$$
\frac{\vartheta_{1}^{\prime}}{\vartheta_{1}}(\nu, \tau)=\frac{\partial}{\partial \nu} \log \vartheta_{1}(\nu, \tau)=\pi \cot (\pi \nu)-4 \pi i \sum_{m, n=1}^{\infty} e^{2 \pi i \tau m n} \sinh (2 \pi i \nu n)
$$


which is valid if $|\exp (2 \pi i(\tau n \pm \nu))|<1$ for all $n \in \mathbb{N}$. Using

$$
\operatorname{coth}(x)=\operatorname{sign}(x)\left[1+2 \sum_{n=1}^{\infty} \exp (-2|x| m)\right]
$$

and extracting the divergence for $t \rightarrow \infty$ stemming from the massless open string modes, one finds

$$
\begin{aligned}
\tilde{\Delta}= & \int_{0}^{\infty} \frac{d t}{t} \frac{\vartheta_{1}^{\prime}}{\vartheta_{1}}\left(\frac{i \theta t}{2}, \frac{i t}{2}\right)+\int_{1}^{\infty} \frac{d t}{t} \pi i \operatorname{sign}(\theta) \\
= & -2 \pi i \operatorname{sign}(\theta) \int_{0}^{\infty} \frac{d t}{t} \sum_{n, m=1}^{\infty}[\exp (-\pi t n(m-1+|\theta|))-\exp (-\pi t n(m-|\theta|))] \\
& -\int_{0}^{1} \frac{d t}{t} \pi i \operatorname{sign}(\theta) \\
= & -2 \pi i \operatorname{sign}(\theta) \sum_{n, m=1}^{\infty} \log \left(\frac{\pi n(m-|\theta|)}{\pi n(m-1+|\theta|)}\right)-\pi i \operatorname{sign}(\theta) \lim _{N \rightarrow \infty} \log N .
\end{aligned}
$$

Clearly, the sum over the positive integers $n$ is divergent, which was expected, as we have not yet subtracted the ultraviolet divergence due to the tadpole. The main observation is that performing a simple zeta-function regularization $\sum_{n=1}^{\infty} 1=\zeta(0)=-\frac{1}{2}$ seems to take care of the tadpole. Indeed, after zeta-function regularization we get

$$
\tilde{\Delta}=\pi i \operatorname{sign}(\theta) \sum_{m=1}^{\infty}\left[\log \left(1-\frac{|\theta|}{m}\right)-\log \left(1-\frac{1-|\theta|}{m}\right)\right]-\pi i \operatorname{sign}(\theta) \lim _{N \rightarrow \infty} \log N .
$$

Using the relations

$$
\log \Gamma(1+x)=-\gamma x+\sum_{k=1}^{\infty}\left[\frac{x}{k}-\log \left(1+\frac{x}{k}\right)\right] \text { and } \gamma=\lim _{N \rightarrow \infty}\left(\sum_{k=1}^{N} \frac{1}{k}-\log N\right)
$$

the last expression becomes

$$
\tilde{\Delta}=\pi i \operatorname{sign}(\theta) \log \left(\frac{\Gamma(|\theta|)}{\Gamma(1-|\theta|)}\right)-2 \pi i \theta \lim _{N \rightarrow \infty} \log N
$$

Finally, performing the sum over $I$ yields:

$$
\begin{aligned}
& T^{A}\left(\mathrm{D} 6_{a}, \mathrm{D} 6_{b}\right)=\frac{I_{a b} N_{b}}{2} \sum_{I=1}^{3} \operatorname{sign}\left(\theta_{a b}^{I}\right) \int_{1}^{\infty} \frac{d t}{t} \\
& \quad-\frac{I_{a b} N_{b}}{2} \log \prod_{I=1}^{3}\left(\frac{\Gamma\left(\left|\theta_{a b}^{I}\right|\right)}{\Gamma\left(1-\left|\theta_{a b}^{I}\right|\right)}\right)^{\operatorname{sign}\left(\theta_{a b}^{I}\right)}+I_{a b} N_{b}\left(\sum_{I=1}^{3} \theta_{a b}^{I}\right) \lim _{N \rightarrow \infty} \log (N) .
\end{aligned}
$$


The last term vanishes due to the supersymmetry condition (4.13). Comparison with 4.28 shows that we are only missing a constant

$$
-\sum_{I=1}^{3} \operatorname{sign}\left(\theta_{a b}^{I}\right)(\log 2-\gamma)
$$

The dependence on the $\theta$ s, however, is correctly reproduced. Thus, indeed, the calculation in the loop channel using zeta-function regularization gives the same result, up to a constant, as the one in the tree channel after removing the divergence that cancels due to the tadpole condition, as has been done above. Zeta-function regularization seems to correctly subtract the divergence due to the closed string tadpole, an observation which we believe to be valuable as a heuristic device. Indeed, we arrived at $(4.19)$ in this way (fixing the constant term by trial and error). Furthermore, the Möbius diagram can also be dealt with by this method if $|\theta|<\frac{1}{2}$.

\section{Regularization for $\mathcal{N}=2$ sectors}

We already remarked above that our results for the $\mathcal{N}=2$ sectors agree with those of [110]. Let us nevertheless sketch a possible regularization of the annulus amplitude. We take as our starting point the raw amplitude [110]

$$
T^{A}\left(\mathrm{D} 6_{a}, \mathrm{D} 6_{b}\right) \propto \int_{0}^{\infty} \frac{d t}{t} \sum_{r, s=-\infty}^{\infty} \exp \left\{-\pi t \frac{\left(r+T_{1}^{I} s\right)^{2}+\left(T_{2}^{I} s\right)^{2}}{V_{I}^{a} T_{2}^{I}}\right\} .
$$

For the duration of this section, we let $T_{1}^{I}$ and $T_{2}^{I}$ be the real, respectively imaginary, parts of the complexified Kähler modulus of that torus $I$ on which the branes lie on top of each other. Moreover, $V_{I}^{a}$ is a constant (see below).

As it stands, 4.42 is both ultraviolet and infrared divergent. The infrared divergence comes from massless states and we take care of it by introducing a small regulating mass $\epsilon \sqrt{V_{I}^{a} T_{2}^{I}}\left(\epsilon \equiv \frac{\mu}{M_{s}}>0\right)$, such that the rhs of 4.42 becomes

$$
\int_{0}^{\infty} \frac{d t}{t} \sum_{r, s=-\infty}^{\infty} \exp \left\{-\pi t \frac{\left(r+T_{1}^{I} s+\epsilon \sqrt{V_{I}^{a} T_{2}^{I}}\right)^{2}+\left(T_{2}^{I} s\right)^{2}}{V_{I}^{a} T_{2}^{I}}\right\} .
$$

To get rid of the ultraviolet divergence (which is cancelled due to tadpole cancellation), we transform to tree channel, upon which 4.43 reads

$$
V_{I}^{a} \int_{0}^{\infty} d l \sum_{r, s=-\infty}^{\infty} \exp \left\{-\frac{\pi l V_{I}^{a}}{T_{2}^{I}}\left[\left(r+T_{1}^{I} s\right)^{2}+\left(T_{2}^{I} s\right)^{2}\right]-2 \pi i s \epsilon \sqrt{V_{I}^{a} T_{2}^{I}}\right\} .
$$


The ultraviolet divergence comes from the state $(r, s)=(0,0)$ [110], which we shall therefore leave out of the summation.

In summary, the regularized amplitude now reads

$$
T^{A}\left(\mathrm{D} 6_{a}, \mathrm{D} 6_{b}\right) \propto V_{I}^{a} \int_{0}^{\infty} d l \sum_{(r, s) \neq(0,0)} \exp \left\{-\frac{\pi l V_{I}^{a}}{T_{2}^{I}}\left[\left(r+T_{1}^{I} s\right)^{2}+\left(T_{2}^{I} s\right)^{2}\right]-2 \pi i s \epsilon \sqrt{V_{I}^{a} T_{2}^{I}}\right\} .
$$

It remains to bring this into a useful form. To this end, one first performs the integral over $l$ and then carries out the sums on $r$ and $s$ (in this order; using Mathematica greatly simplifies the task). Thereafter, one looks at the resulting expression in the limit of very small $\epsilon$, extracting regular and singular pieces.

The result is that in a sector preserving $\mathcal{N}=2$ supersymmetry one finds, in accordance with [110] (recall that $\epsilon=\frac{\mu}{M_{s}}$ ):

$$
T^{A}\left(\mathrm{D} 6_{a}, \mathrm{D} 6_{b}\right)=N_{b}\left|I_{a b}^{J} I_{a b}^{K}\right|\left[\log \left(\frac{M_{s}^{2}}{\mu^{2}}\right)-\log \left|\eta\left(i T_{I}^{c}\right)\right|^{4}-\log \left(T_{I} V_{I}^{a}\right)+C\right],
$$

where $I$ denotes the torus on which the branes lie on top of each other, $T_{I} \equiv T_{1}^{I}$ is the torus' Kähler modulus, and $T_{I}^{c} \equiv T_{1}^{I}+i T_{2}^{I}$ its complexification. Furthermore, we have defined $V_{I}^{a}=\left|n_{a}^{I}+i u_{I} m_{a}^{I}\right|^{2} / u_{I}$, with $u_{I}$ the complex structure modulus of the torus and $n_{a}^{I}, m_{a}^{I}$ the wrapping numbers of brane stack $a$. In the regularization scheme sketched here, the numerical constant $C=-\log 4 \pi^{2}$. However, this clearly is not universal, as for e.g. dimensional regularization [110] one obtains $C=\gamma_{E}-\log (4 \pi)$. In any case, we need not worry about this here as for our purposes later on the term of most interest in 4.46 is the moduli dependent piece $\log \left|\eta\left(i T_{I}^{c}\right)\right|^{4}$ and this clearly is independent of such issues.

\section{Discussion}

We already mentioned that for the $\mathcal{N}=1$ sectors our results are slightly different than those of [110]. In order to compare with that work, it is useful to specialize to $\theta_{a b}^{1,2}>0$, $\theta_{a b}^{3}<0$. Equation 4.28 then contains the following threshold corrections

$$
\Delta_{a}=-\frac{b_{a}}{16 \pi^{2}} \log \left[\frac{\Gamma\left(\theta_{a b}^{1}\right) \Gamma\left(\theta_{a b}^{2}\right) \Gamma\left(1+\theta_{a b}^{3}\right)}{\Gamma\left(1-\theta_{a b}^{1}\right) \Gamma\left(1-\theta_{a b}^{2}\right) \Gamma\left(-\theta_{a b}^{3}\right)}\right]
$$

$\left(b_{a}=\frac{I_{a b} N_{b}}{2}\right)$, which are to be compared with [110]

$$
\tilde{\Delta}_{a}=-\frac{b_{a}}{16 \pi^{2}} \log \left[\frac{\Gamma\left(1+\theta_{a b}^{1}\right) \Gamma\left(1+\theta_{a b}^{2}\right) \Gamma\left(1+\theta_{a b}^{3}\right)}{\Gamma\left(1-\theta_{a b}^{1}\right) \Gamma\left(1-\theta_{a b}^{2}\right) \Gamma\left(1-\theta_{a b}^{3}\right)}\right] .
$$

Clearly, $\Delta_{a}$ and $\tilde{\Delta}_{a}$ are not identical, the difference being

$$
\Delta_{a}-\tilde{\Delta}_{a}=-\frac{b_{a}}{16 \pi^{2}} \log \left[-\frac{\theta_{a b}^{3}}{\theta_{a b}^{1} \theta_{a b}^{2}}\right]
$$




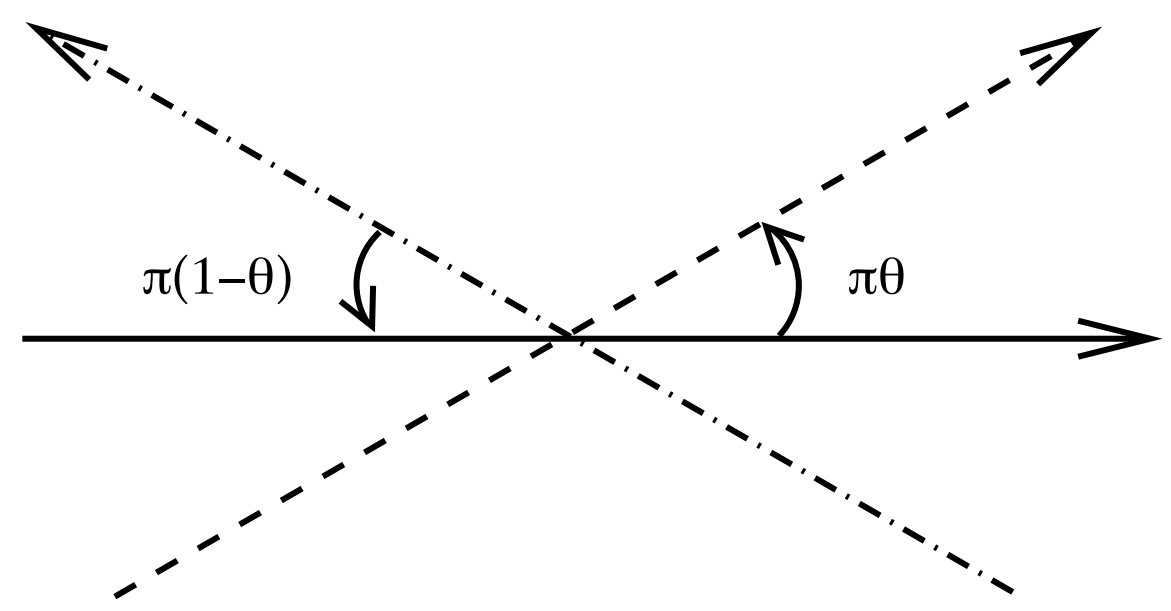

Figure 4.2: This figure shows two branes. In one configuration they intersect at an angle $\pi \theta$, while in the other they intersect at an angle $\pi(1-\theta)$. The regularized threshold corrections should respect the obvious symmetry of these situations.

This difference appears to stem from the different treatment of open string states in the threshold corrections, which are located at the intersection of two D6-branes and whose masses are proportional to an integer multiple of the intersection angle $\theta_{a b}^{I}$. These states are in fact included in the threshold corrections $\Delta_{a}$. For small intersection angles some of these states become lighter than the string scale $M_{s}$, and hence $\Delta_{a}$ logarithmically diverges for $\theta_{a b}^{I} \rightarrow 0$. On the other hand, $\tilde{\Delta}_{a}$ is completely regular for $\theta_{a b}^{I} \rightarrow 0$, because it does not contain the contribution of these states that become light when $\left.\theta_{a b}^{I} \rightarrow 0\right]^{6}$ In more technical terms, this different behavior can be traced back to how the infrared divergences were treated during the computation of the threshold corrections. In our results, the contribution of the massless modes appears in the logarithmic running of the gauge coupling constant, whereas the contribution of the modes that become light for $\theta_{a b}^{I} \rightarrow 0$ is kept in $\Delta_{a}$. This is in contrast to the infrared regularization method employed in [110] for the computation of $\tilde{\Delta}_{a}$, where also the contribution of the modes with masses proportional to $m \theta_{a b}^{I}, m \in \mathbb{N}$ is subtracted from the threshold corrections.

There is another, more intuitive, reason to have faith in the results presented here. The original, divergent expression for the annulus diagram (4.12) is formally antisymmetric under the replacement $\theta_{a b}^{I} \rightarrow 1-\theta_{a b}^{I}$. Therefore, we should expect this to be the case for the regularized result as well. In geometric terms, this antisymmetry just encodes the obvious symmetry of the situation in figure 4.2. Now, our result (4.47) shows this kind of antisymmetry (cf. figure 4.3), whereas the result (4.48) does not (cf. figure 4.4).

Finally, let us remark that the one-loop correction $\Delta_{a}$ to the gauge coupling constant is not the real part of a holomorphic function when expressed in terms of the complex structure moduli fields $U^{I}$ of the underlying torus $T^{6}$, since the intersection angles $\theta_{a b}^{I}$ are

\footnotetext{
${ }^{6}$ Note, however, that both $\Delta_{a}$ and $\tilde{\Delta}_{a}$ contain the contribution of states that become light for $\theta_{a b}^{I} \rightarrow 1$.
} 


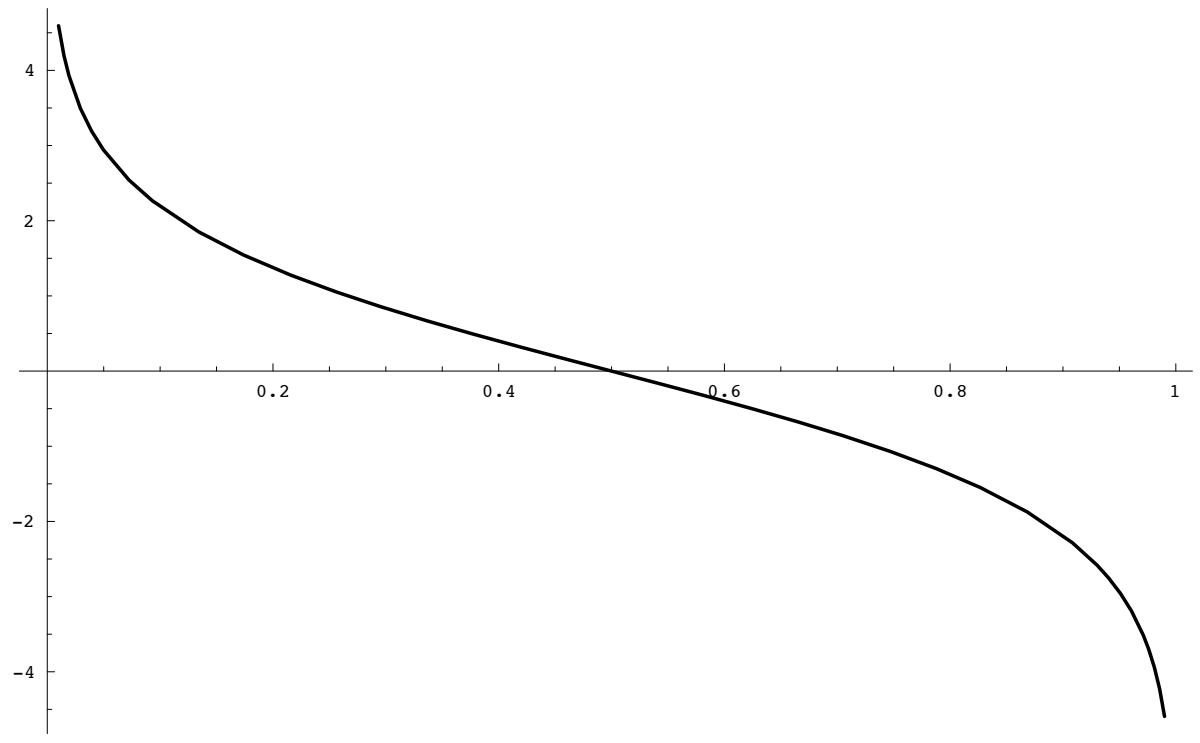

Figure 4.3: Plot of the threshold corrections $\Delta_{a}$ versus $\theta$ for the section $\theta_{a b}^{1}=\theta, 2 \theta_{a b}^{1}=$ $2 \theta_{a b}^{2}=-\theta_{a b}^{3}$.

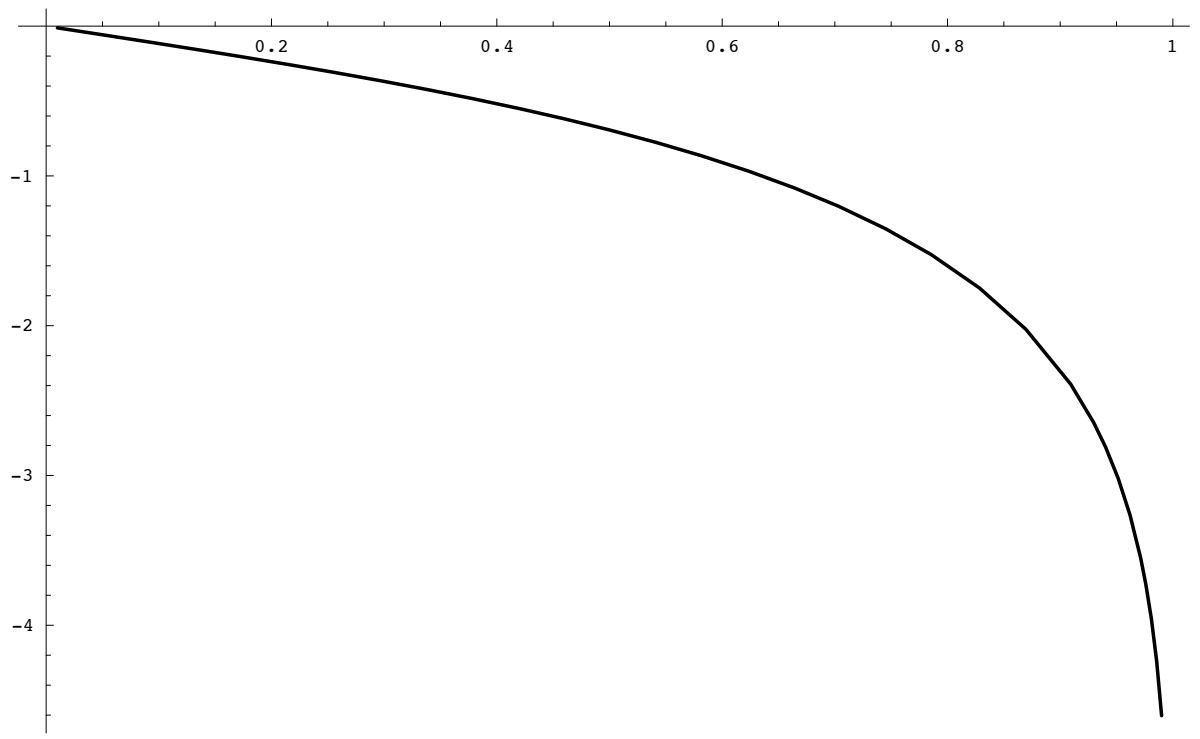

Figure 4.4: Plot of the threshold corrections $\tilde{\Delta}_{a}$ versus $\theta$ for the section $\theta_{a b}^{1}=\theta, 2 \theta_{a b}^{1}=$ $2 \theta_{a b}^{2}=-\theta_{a b}^{3}$. 
non-holomorphic functions of the $U^{I}$. We examine this further in the next section.

\subsection{Extracting $f_{a}^{1-l o o p}$ from Gauge Threshold Correc- tions}

In the previous section we saw that in general the gauge threshold corrections in intersecting D6-brane models are non-holomorphic functions of the closed string moduli. More explicitly, they are the sum of a non-holomorphic piece and the real part of a holomorphic function:

$$
\Delta_{a}=\Delta_{a}^{\text {n.h. }}+\operatorname{Re}\left(\Delta_{a}^{\text {hol. }}\right)
$$

We now take up the question how we can extract the one-loop corrections to the gauge kinetic functions from gauge threshold corrections. At first sight this problem appears illposed, as the gauge kinetic functions are holomorphic. Nevertheless, there ought to exist some such connection since the gauge kinetic functions enter into the low-enery supergravity more or less as couplings. To proceed, we must first understand how the non-holomorphic parts of the gauge threshold corrections arise.

The way to understand this is to refer to the so-called Kaplunovsky-Louis formula. Namely, in a supersymmetric gauge theory one can compute the running gauge couplings $g_{a}\left(\mu^{2}\right)$ in terms of the gauge kinetic functions $f_{a}$, the Kähler potential $\mathcal{K}$ and the Kähler metrics of the charged matter fields $K^{a b}\left(\mu^{2}\right)$ via the equation [129, 61, 60, 104, 85, 93, 94]:

$$
\begin{aligned}
\frac{8 \pi^{2}}{g_{a}^{2}\left(\mu^{2}\right)}=8 \pi^{2} \operatorname{Re}\left(f_{a}\right) & +\frac{b_{a}}{2} \log \left(\frac{M_{P}^{2}}{\mu^{2}}\right)+\frac{c_{a}}{2} \mathcal{K}+ \\
& +T\left(G_{a}\right) \log g_{a}^{-2}\left(\mu^{2}\right)-\sum_{r} T_{a}(r) \log \operatorname{det} K_{r}\left(\mu^{2}\right) .
\end{aligned}
$$

Here,

$$
b_{a}=\sum_{r} n_{r} T_{a}(r)-3 T\left(G_{a}\right), \quad c_{a}=\sum_{r} n_{r} T_{a}(r)-T\left(G_{a}\right)
$$

and $T_{a}(r)=\operatorname{Tr}\left(T_{(a)}^{2}\right)$, where the $T_{(a)}$ are the generators of the gauge group $G_{a}$. In addition, $T\left(G_{a}\right)=T_{a}\left(\operatorname{adj} G_{a}\right)$ and $n_{r}$ is the number of multiplets in the representation $r$ of the gauge group. The sums run over these representations. Note that in 4.51) the Planck scale shows up as the natural cutoff scale.

Equation (4.51) is to be understood recursively, in the sense that in order to calculate the one-loop running of the coupling one has to insert the tree-level results for the last three terms. 
Note that the right hand side of 4.51$)$ is a mix of holomorphic $\left(f_{a}\right)$ and non-holomorphic pieces $\left(\mathcal{K}, K^{a b}\right)$. The left hand side of (4.51) is given by equation (4.9), which for convenience we repeat here:

$$
\frac{8 \pi^{2}}{g_{a}^{2}(\mu)}=\frac{8 \pi^{2}}{g_{a, \text { string }}^{2}}+\frac{b_{a}}{2} \log \left(\frac{M_{s}^{2}}{\mu^{2}}\right)+\frac{\Delta_{a}}{2} .
$$

On the right hand side of (4.51), $f_{a}$ denotes the Wilsonian, i.e. holomorphic, gauge kinetic function. Comparing 4.53) and (4.51) while taking into account the non-renormalization theorem for the gauge kinetic function (4.7) suggests the relation

$$
f_{a}^{1-\text { loop }}\left(e^{-T_{i}^{c}}\right)=\Delta_{a}^{\text {hol. }} .
$$

In fact, this is the correct formula. Showing this for the example of intersecting D6-brane models on the $\mathbb{Z}_{2} \times \mathbb{Z}_{2}$ toroidal orbifold requires matching the right hand sides of (4.51) and (4.53), which we shall do in the next section. In addition, we remark that the same sort of calculation has also been carried out in [37] for intersecting D6-brane models on the $\mathbb{Z}_{2} \times \mathbb{Z}_{2}^{\prime}$ toroidal orbifold.

\subsubsection{Results for $T^{6} / \mathbb{Z}_{2} \times \mathbb{Z}_{2}$ Models}

In this section we carry out the extraction of the one-loop corrections to the gauge kinetic functions from gauge threshold corrections for $T^{6} / \mathbb{Z}_{2} \times \mathbb{Z}_{2}$ intersecting D6-brane models. However, as the formulas are slightly involved, it might be useful to summarize the results beforehand:

- One-loop corrections to the gauge kinetic function in $\mathcal{N}=1$ sectors. The gauge threshold corrections in the $\mathcal{N}=1$ sectors are (non-holomorphic) functions of the $U_{I}^{c}$ alone, cf. (4.28), 4.30), and (4.47). Therefore, by (4.54), the gauge kinetic functions receive no one-loop corrections from these sectors:

$$
\mathcal{N}=1 \text { sectors : } f_{a}^{1 \text { loop }}=0 .
$$

- One-loop corrections to the gauge kinetic function in $\mathcal{N}=2$ sectors.

For the $\mathcal{N}=2$ sectors, the gauge threshold corrections 4.46 contain the term $\log \left|\eta\left(i T_{I}^{c}\right)\right|^{4}$, and this can be written as the real part of a holomorphic function:

$$
\log \left|\eta\left(i T_{I}^{c}\right)\right|^{4}=4 \operatorname{Re}\left[\log \eta\left(i T_{I}^{c}\right)\right] .
$$

Including all prefactors gives the one-loop correction

$$
\mathcal{N}=2 \text { sectors : } \quad f_{a}^{1 \text {-loop }}=-\sum_{b} \frac{N_{b}\left|I_{a b}^{J} I_{a b}^{K}\right|}{4 \pi^{2}} \log \eta\left(i T_{I}^{c}\right),
$$

where $I \neq J \neq K \neq I$ and the sum only runs over brane stacks $b$ which lie on top of the stack $a$ in exactly one torus, denoted by $I$. 
In order to carry out the matching of the right hand sides of 4.51) and 4.53), one first has to relate the different scales $M_{P}$ and $M_{s}$ in those formulas. This is done by using

$$
\frac{M_{s}^{2}}{M_{P}^{2}} \propto \exp \left(2 \phi_{4}\right) \propto\left(S U_{1} U_{2} U_{3}\right)^{-\frac{1}{2}}
$$

where $\phi_{4}$ is the four-dimensional dilaton (see below (4.2p) and the complex structure moduli in the supergravity basis can be expressed in terms of $\phi_{4}$ and $u_{I}=R_{I, 2} / R_{I, 1}$, where the $R$ 's are the radii of the I'th torus, as [109]

$$
S=\frac{1}{2 \pi} e^{-\phi_{4}} \frac{1}{\sqrt{u_{1} u_{2} u_{3}}}, \quad U_{I}=\frac{1}{2 \pi} e^{-\phi_{4}} \sqrt{\frac{u_{J} u_{K}}{u_{I}}}, \quad \text { with } I \neq J \neq K \neq I .
$$

These fields are the real parts of complex scalars $S^{c}$ and $U_{I}^{c}$ of four dimensional chiral multiplets.

Since $\mathcal{N}=4$ super Yang-Mills theory is finite, one expects the sum of the terms in (4.51) proportional to $T\left(G_{a}\right)$ to cancel. This is because the only chiral multiplets transforming in the adjoint representation of the gauge group are the open string moduli which (on the background considered) assemble themselves into three chiral multiplets, thus forming an $\mathcal{N}=4$ sector together with the gauge fields.

To show that this cancellation indeed happens, one notices the following. Firstly, $n_{\text {adjoint }}=$ 3 , as explained, such that there is no term in $b_{a}$ proportional to $T\left(G_{a}\right)$. Secondly [12, 109],

$$
\begin{aligned}
\mathcal{K} & =-\log \left(S^{c}+\bar{S}^{c}\right)-\sum_{I=1}^{3} \log \left(U_{I}^{c}+\bar{U}_{I}^{c}\right)-\sum_{I=1}^{3} \log \left(T_{I}^{c}+\bar{T}_{I}^{c}\right) \\
g_{a, \text { tree }}^{-2} & =S \prod_{I=1}^{3} n_{a}^{I}-\sum_{I=1}^{3} U_{I} n_{a}^{I} m_{a}^{J} m_{a}^{K} \quad I \neq J \neq K \neq I,
\end{aligned}
$$

where $T_{I}$ are the Kähler moduli of the torus and $n_{a}^{I}, m_{a}^{I}$ are the wrapping numbers of the brane. Finally, one needs the matter metric for the open string moduli which reads [35] (cf. also [9])

$$
K_{i j}^{I}=\frac{\delta_{i j}}{T_{I} U_{I}}\left|\frac{\left(n_{a}^{J}+i u_{J} m_{a}^{J}\right)\left(n_{a}^{K}+i u_{K} m_{a}^{K}\right)}{\left(n_{a}^{I}+i u_{I} m_{a}^{I}\right)}\right| \quad I \neq J \neq K \neq I,
$$

for $I=1, \ldots, 3$.

Let us now turn to the fields in the fundamental representation of the gauge group $G_{a}$ on brane stack $a$, in particular to the fields arising from the intersection with one other stack of branes, denoted by $b$. For an $\mathcal{N}=1$ open string sector, the metric for these fields can be written as [109, 35] (see also [101])

$$
K_{i j}^{a b}=\delta_{i j} S^{-\alpha} \prod_{I=1}^{3} U_{I}^{-\left(\beta+\xi \theta_{a b}^{I}\right)} T_{I}^{-\left(\gamma+\zeta \theta_{a b}^{I}\right)} \sqrt{\frac{\Gamma\left(\theta_{a b}^{1}\right) \Gamma\left(\theta_{a b}^{2}\right) \Gamma\left(1+\theta_{a b}^{3}\right)}{\Gamma\left(1-\theta_{a b}^{1}\right) \Gamma\left(1-\theta_{a b}^{2}\right) \Gamma\left(-\theta_{a b}^{3}\right)}},
$$


where $\alpha, \beta, \gamma, \xi$ and $\zeta$ are undetermined constants. As $\theta_{a b}^{1,2}>0$ and $\theta_{a b}^{3}<0$, which is assumed in (4.63), the intersection number $I_{a b}$ is positive, implying that

$$
n_{f}=I_{a b} N_{b} .
$$

Using $T_{a}(f)=\frac{1}{2}$ and relations $4.52,4.58,4.60,4.63,4.64$, one finds a contribution to the right hand side of (4.51) proportional to

$$
\begin{aligned}
& \frac{I_{a b} N_{b}}{2}\left(\log \left(\frac{M_{s}^{2}}{\mu^{2}}\right)+(2 \gamma-1) \log \left(T_{1} T_{2} T_{3}\right)+\left(2 \beta-\frac{1}{2}\right) \log \left(U_{1} U_{2} U_{3}\right)+\left(2 \alpha-\frac{1}{2}\right) \log S\right. \\
& \left.+\zeta \sum_{I=1}^{3} \theta_{a b}^{I} \log T_{I}+\xi \sum_{I=1}^{3} \theta_{a b}^{I} \log U_{I}-\log \left[\frac{\Gamma\left(\theta_{a b}^{1}\right) \Gamma\left(\theta_{a b}^{2}\right) \Gamma\left(1+\theta_{a b}^{3}\right)}{\Gamma\left(1-\theta_{a b}^{1}\right) \Gamma\left(1-\theta_{a b}^{2}\right) \Gamma\left(-\theta_{a b}^{3}\right)}\right]\right) .
\end{aligned}
$$

Using 4.47) one sees that the first and the last term exactly reproduce the contribution of the last two terms in (4.53). It can be shown [9] that the terms proportional to $\zeta$ and $\xi$ constitute a universal gauge coupling correction (see below). The remaining three terms can neither be attributed to such a correction nor can they be written as the real part of a holomorphic function. Thus they cannot be the one-loop correction to the gauge kinetic function and therefore must vanish. This fixes some of the coefficients in the ansatz (4.63):

$$
\alpha=\beta=\frac{1}{4}, \quad \gamma=\frac{1}{2} .
$$

The same matching of terms appears between the Möbius diagram plus the annulus with boundaries on brane $a$ and its orientifold image and the Kähler metrics for fields in the symmetric and antisymmetric representation. Here, one has to replace $\theta_{a b}^{I}$ and $I_{a b} N_{b}$ by $\theta_{a a^{\prime}}^{I}=2 \theta_{a}^{I}$ and $I_{a a^{\prime}} N_{a}$ in (4.63) and 4.13). Apart from these replacements, the Kähler metric for matter in these representations is also given by (4.63) with the constants $\alpha, \beta$, $\gamma$ given in 4.66).

The corrections to the gauge couplings coming from $\mathcal{N}=2$ open string sectors were seen in section 4.3.1 to take on quite a different form. They contain a term,

$$
-\log \left|\eta\left(i T_{I}^{c}\right)\right|^{4}=-4 \operatorname{Re}\left[\log \eta\left(i T_{I}^{c}\right)\right]
$$

which can be written as the real part of a holomorphic function. This leads one to conclude that the gauge kinetic function receives one-loop corrections from these sectors. Inserting the correct prefactor, which from the first term in 4.46) and the corresponding one in (4.51) can be seen to be proportional to the beta function coefficient, gives

$$
f_{a}^{1 \text {-loop }}=-\frac{N_{b}\left|I_{a b}^{J} I_{a b}^{K}\right|}{4 \pi^{2}} \log \eta\left(i T_{I}^{c}\right) \quad I \neq J \neq K \neq I,
$$

where again $I$ denotes the torus in which the branes lie on top of each other and $I_{a b}^{J, K}$ are the intersection numbers on the other tori. 
The term $-\log \left(T_{I} V_{I}^{a}\right)$ in 4.46$)$ is not the real part of a holomorphic function. Proceeding as before, one finds that the Kähler metric for the hypermultiplet (or two chiral multiplets) living at an intersection of branes $a$ and $b$ preserving eight supercharges must be

$$
K_{i j}^{I}=\frac{\left|n_{a}^{I}+i u_{I} m_{a}^{I}\right|}{\left(U^{J} U^{K} T^{J} T^{K}\right)^{\frac{1}{2}}} \quad I \neq J \neq K \neq I .
$$

Apart from the factor in the numerator, this is in agreement with the form found by direct calculations [109, 35]. The appearance of the numerator is however plausible as it also appears in the open string moduli metric and the hypermultiplets under discussion should feel the $I$ 'th torus in the same way.

\section{Universal gauge coupling corrections}

We remarked above that in the process of matching (4.51) and 4.53) one finds that the treelevel gauge coupling (4.8) receives a gauge group independent correction. Such corrections also appear in the heterotic [94] and type I [11, 25] string and are related to a redefinition of the dilaton at one-loop [60, 61]. Here we shall only take note of the result, and refer to [9] for details. It is

$$
g_{a, \text { string }}^{-2}=S^{L} \prod_{I=1}^{3} n_{a}^{I}-\sum_{I=1}^{3} U_{I}^{L} n_{a}^{I} m_{a}^{J} m_{a}^{K}
$$

with redefined dilaton and complex structure moduli

$$
\begin{aligned}
S^{L} & =S-\frac{1}{8 \pi^{2}} \sum_{b} N_{b} m_{b}^{1} m_{b}^{2} m_{b}^{3} \sum_{I=1}^{3} \phi_{b}^{I} \zeta^{\prime} \log T_{I} \\
U_{J}^{L} & =U_{J}+\frac{1}{8 \pi^{2}} \sum_{b} N_{b} m_{b}^{J} n_{b}^{K} n_{b}^{L} \sum_{I=1}^{3} \phi_{b}^{I} \zeta^{\prime} \log T_{I},
\end{aligned}
$$

where $J \neq K \neq L \neq J$ and $\zeta^{\prime}= \pm \frac{1}{2}$. The appearance of these redefined fields is closely related to the cancellation of supergravity sigma-model anomalies. Incidentally, related to the universal corrections, the remaining constants $(\xi, \zeta)$ in the $\mathcal{N}=1$ matter Kähler metrics 4.63) are also fixed, so that

$$
K_{i j}^{a b}=\delta_{i j} S^{-\frac{1}{4}} \prod_{J=1}^{3} U_{J}^{-\frac{1}{4}} T_{J}^{-\left(\frac{1}{2} \pm \frac{1}{2} \operatorname{sign}\left(I_{a b}\right) \theta_{a b}^{J}\right)} \sqrt{\frac{\Gamma\left(\theta_{a b}^{1}\right) \Gamma\left(\theta_{a b}^{2}\right) \Gamma\left(1+\theta_{a b}^{3}\right)}{\Gamma\left(1-\theta_{a b}^{1}\right) \Gamma\left(1-\theta_{a b}^{2}\right) \Gamma\left(-\theta_{a b}^{3}\right)}},
$$

where $\sum_{I=1}^{3} \theta_{a b}^{I}=0$. (This result is not of the most general form as it is subject to the restriction $\theta_{a b}^{1}, \theta_{a b}^{2}>0, \theta_{a b}^{3}<0$.) 


\section{Chapter 5}

\section{D-instanton Corrections to Low Energy Effective Couplings ${ }^{1}$}

\footnotetext{
${ }^{1}$ This chapter is based, sometimes verbatim, on the papers [9, 7] together with R. Blumenhagen, D. Lüst, and M. Schmidt-Sommerfeld.
} 


\subsection{Introduction}

In chapter 4 we looked at the one-loop corrections that the gauge kinetic functions in the low-energy effective action for intersecting D6-brane models can receive. In particular, we showed how the holomorphic one-loop corrections to the gauge kinetic functions can be extracted from the non-holomorphic gauge threshold corrections.

Now, the non-renormalization theorems of that chapter left open the possibility that the gauge kinetic function can receive non-perturbative corrections. Indeed, spacetime instanton corrections in intersecting D6-brane models come from E2-instantons and we shall investigate in section 5.3 under which conditions we might expect corrections the gauge kinetic functions.

However, first we will be concerned (again) with instanton contributions to the superpotential. The structure of the charged matter field superpotential instanton calculus [34] is such that certain instantonic one-loop amplitudes appear as integrands in the contributions of E2-instantons to the couplings of the charged matter fields. Since it ought to be possible to incorporate these couplings into a superpotential, it is necessary to show precisely how to extract the holomorphic contributions from the instanton calculus. This we do in section 5.2 .

\section{$O(1)$ Instantons}

Below we will be concerned with E2-instanton corrections to the superpotential and gauge kinetic functions in intersecting D6-brane models. Recall that an E2-instanton is a (fictitious) three-dimensional brane whose world-volume is wrapped around a three-cycle in the compactification manifold. In principle, for any given compactification, there are many instantons but only those preserving half of the $\mathcal{N}=1$ supersymmetry can contribute to the holomorphic superpotential and gauge kinetic functions. For the instanton calculus zero mode measure this means that it must contain a factor $d^{4} x d^{2} \theta$ (cf. (3.10)).

As has been explained in [27, 86], an E2-instanton wrapped around a rigid special Lagrangian three-cycle generically gives rise to four bosonic zero modes $x^{\mu}$ and four fermionic ones $\theta^{1,2}, \bar{\theta}^{1,2}$. However, for an instanton wrapping a cycle invariant under the orientifold projection, the situation is different:

For so-called $O(n)$ instantons the zero modes $x^{\mu}, \theta$ are symmetrized and the mode $\bar{\theta}$ gets anti-symmetrized. For the opposite projection, i.e. for $U S p(2 n)$ instantons, the zero modes $x^{\mu}, \theta$ are anti-symmetrized and the mode $\bar{\theta}$ gets symmetrized.

Thus, the zero mode measure $d^{4} x d^{2} \theta$ can be obtained for a single $O(1)$ instanton [17]. We will restrict our attention to such instantons when looking at instanton corrections to the superpotential and gauge kinetic functions. Note, however, that $O(1)$ instantons are not necessarily the only instantons of interest [117, 54]. 


\subsection{Contributions to the Superpotential}

In this section we look again at E2-instanton contributions to the superpotential in intersecting D6-brane models, this time slightly more 'formally'. We will see that the instanton calculus proposed in 34] features manifestly non-holomorphic terms. However, the superpotential is a holomorphic quantity and thus it is clearly necessary to understand how to extract the holomorphic pieces.

We are looking at the contribution of a single E2-instanton to the superpotential. In line with the introductory remarks above, we restrict our attention to a single $O(1)$ E2instanton wrapping the cycle $\Xi$. This will give us the zero modes $x^{\mu}$ and $\theta^{\alpha}$. In order for the E2-instanton to contribute to the superpotential, we also require that in our model there do not arise any additional zero modes from strings starting and ending on it. This means that $\Xi$ must be rigid, i.e. that we have $b_{1}(\Xi)=0$.

Additional zero modes can only arise from the intersection of the instanton $\Xi$ with D6branes $\Pi_{a}$. There are $N_{a}\left[\Xi \cap \Pi_{a}\right]^{+}$chiral fermionic zero modes $\lambda_{a, I}$ and $N_{a}\left[\Xi \cap \Pi_{a}\right]^{-}$ anti-chiral ones, $\bar{\lambda}_{a, J} \stackrel{2}{2}^{2}$

In chapter 3 we computed the superpotential contribution of the E2-instanton 'directly', but here we shall look at the original proposal [34, which computes correlators of charged matter fields in the presence of the instanton. The question of holomorphy of the superpotential in this approach reduces to the holomorphy of Yukawa couplings.

For the presentation of the calculus we recall the short-hand notation

$$
\widehat{\Phi}_{a_{k}, b_{k}}\left[\vec{x}_{k}\right]=\Phi_{a_{k}, x_{k, 1}} \cdot \Phi_{x_{k, 1}, x_{k, 2}} \cdot \Phi_{x_{k, 2}, x_{k, 3}} \cdot \ldots \cdot \Phi_{x_{k, n-1}, x_{k, n}} \cdot \Phi_{x_{k, n(k)}, b_{k}}
$$

for the chain-product of open string vertex operators, with the definition $\widehat{\Phi}_{a_{k}, b_{k}}[\overrightarrow{0}]=\Phi_{a_{k}, b_{k}}$.

In the CFT which goes with our intersecting D6-brane model we can compute correlators of charged matter fields $\Phi$ in physical normalization, combining the Yukawa couplings $Y$ with the matter field Kähler potential $\mathcal{K}$ and metrics $K_{a b}$ :

$$
\left\langle\Phi_{a_{1}, b_{1}} \cdot \ldots \cdot \Phi_{a_{M}, b_{M}}\right\rangle_{\mathrm{E} 2}=\frac{e^{\frac{\mathcal{K}}{2}} Y_{\Phi_{a_{1}, b_{1}}, \ldots, \Phi_{a_{M}, b_{M}}}}{\sqrt{K_{a_{1}, b_{1}} \cdot \ldots \cdot K_{a_{M}, b_{M}}}} .
$$

In [34] a general expression for the left hand side of (5.2) was proposed, instructing us to evaluate the following zero mode integral over disk and one-loop open string CFT amplitudes

$$
\begin{gathered}
\left\langle\Phi_{a_{1}, b_{1}} \cdot \ldots \cdot \Phi_{a_{M}, b_{M}}\right\rangle_{\mathrm{E} 2}=\frac{V_{3}}{g_{s}} \int d^{4} x d^{2} \theta \sum_{\text {conf. }} \prod_{a}\left(\prod_{i=1}^{\left[\Xi \cap \Pi_{a}\right]^{+}} d \lambda_{a}^{i}\right)\left(\prod_{i=1}^{\left[\Xi \cap \Pi_{a}\right]^{-}} d \bar{\lambda}_{a}^{i}\right) \\
\exp \left(-S_{\mathrm{E} 2}\right) \exp \left(Z_{0}^{\prime}(\mathrm{E} 2)\right)\left\langle\widehat{\Phi}_{a_{1}, b_{1}}\left[\vec{x}_{1}\right]\right\rangle_{\lambda_{a_{1}}, \bar{\lambda}_{b_{1}}} \cdot \ldots \cdot\left\langle\widehat{\Phi}_{a_{L}, b_{L}}\left[\vec{x}_{L}\right]\right\rangle_{\lambda_{a_{L}}, \bar{\lambda}_{b_{L}}} .
\end{gathered}
$$

\footnotetext{
${ }^{2}$ The physical intersection number $\Pi_{a} \cap \Pi_{b}$ between two cycles $\Pi_{a}$ and $\Pi_{b}$ is the sum of positive $\left[\Pi_{a} \cap \Pi_{b}\right]^{+}$and negative $\left[\Pi_{a} \cap \Pi_{b}\right]^{-}$intersections.
} 


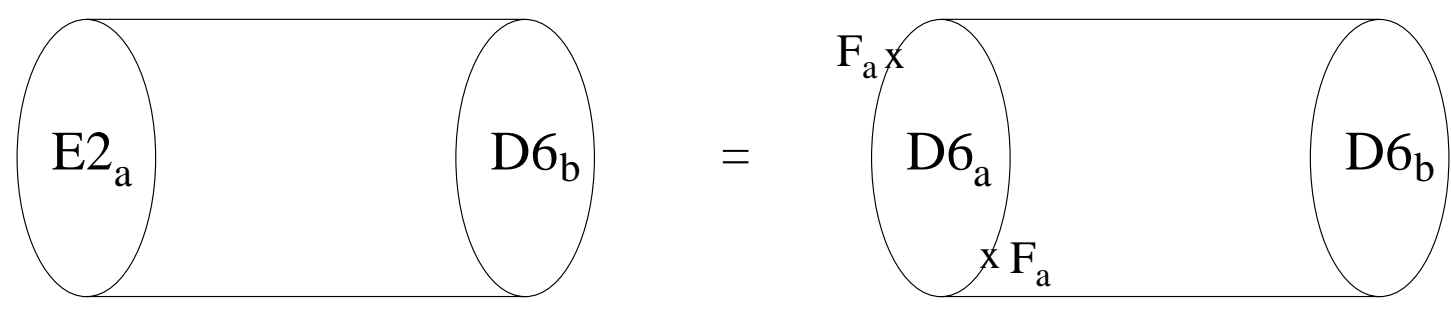

Figure 5.1: Relation between instantonic annulus amplitudes and corresponding gauge threshold corrections

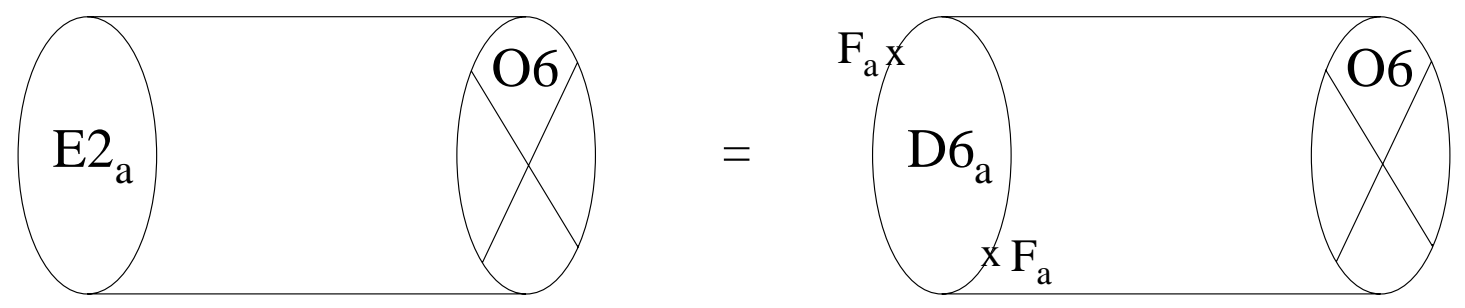

Figure 5.2: Relation between instantonic Möbius amplitude and corresponding gauge threshold corrections

For simplicity, we do not consider the case where matter fields are also assigned to string loop diagrams. Here $Z_{0}^{\prime}$ consists of annulus diagrams for open strings with one boundary on the E2-instanton and the other boundary on the various D6-branes, as well as Möbius diagrams with their boundary on the E2-instanton:

$$
Z_{0}^{\prime}(\mathrm{E} 2)=\sum_{b} Z^{\prime A}\left(\mathrm{E} 2_{a}, \mathrm{D} 6_{b}\right)+Z^{\prime M}\left(\mathrm{E} 2_{a}, \mathrm{O} 6\right)
$$

The prime is to indicate that we only sum over the massive open string states in the loop amplitude, as the zero modes are taken care of explicitly in (5.3).

The annulus and Möbius diagrams in (5.4) are equal to gauge threshold corrections, see figure 5.1 and figure 5.2, which holds for the even spin structures.

To prove the first statement, let us compute the instantonic annulus one-loop partition functions $Z^{A}\left(\mathrm{E} 2_{a}, \mathrm{D} 6_{b}\right)$ (see also [1]). These cannot be expressed in light cone gauge, but including the contributions from the ghosts can, for the even spin structures, be written as

$$
Z^{A}\left(\mathrm{E} 2_{a}, \mathrm{D} 6_{b}\right)=-\int_{0}^{\infty} \frac{d t}{t} \sum_{\alpha, \beta \neq\left(\frac{1}{2}, \frac{1}{2}\right)}(-1)^{2(\alpha+\beta)} \frac{\vartheta^{2}\left[\begin{array}{c}
\alpha \\
\beta
\end{array}\right](i t, i t / 2)}{\vartheta^{2}\left[\begin{array}{l}
1 / 2 \\
1 / 2
\end{array}\right](i t, i t / 2)} \frac{\eta^{3}(i t)}{\vartheta\left[\begin{array}{c}
\alpha \\
\beta
\end{array}\right](i t, 0)} A_{a b}\left[\begin{array}{c}
\alpha \\
\beta
\end{array}\right]
$$

with

$$
\vartheta\left[\begin{array}{l}
\alpha \\
\beta
\end{array}\right](\tau, z)=\sum_{n \in \mathbb{Z}} e^{i \pi \tau(n+\alpha)^{2}} e^{2 \pi i(n+\alpha)(z+\beta)}
$$


Moreover, $A_{a b}\left[\begin{array}{l}\alpha \\ \beta\end{array}\right]$ denotes the internal open string partition function with the respective spin-structure for open strings between branes wrapping the three-cycles $\Pi_{a}$ and $\Pi_{b}$.

Using the relations

$$
\left(\frac{\vartheta\left[\begin{array}{l}
\alpha \\
\beta
\end{array}\right](i t, z) \vartheta^{\prime}\left[\begin{array}{l}
1 / 2 \\
1 / 2
\end{array}\right](i t, 0)}{\vartheta\left[\begin{array}{l}
1 / 2 \\
1 / 2
\end{array}\right](i t, z) \vartheta\left[\begin{array}{c}
\alpha \\
\beta
\end{array}\right](i t, 0)}\right)^{2}=\frac{\vartheta^{\prime \prime}\left[\begin{array}{l}
\alpha \\
\beta
\end{array}\right](i t, 0)}{\vartheta\left[\begin{array}{c}
\alpha \\
\beta
\end{array}\right](i t, 0)}-\partial_{z}^{2} \log \vartheta\left[\begin{array}{l}
1 / 2 \\
1 / 2
\end{array}\right](i t, z),
$$

where the derivatives are taken with respect to the variable $z$, and $-2 \pi \eta^{3}(i t)=\vartheta^{\prime}\left[\begin{array}{l}1 / 2 \\ 1 / 2\end{array}\right](i t, 0)$, after a few standard manipulations we can bring the partition function into the form

$$
Z^{A}\left(\mathrm{E} 2_{a}, \mathrm{D} 6_{b}\right)=\int_{0}^{\infty} \frac{d t}{t} \sum_{\alpha, \beta \neq\left(\frac{1}{2}, \frac{1}{2}\right)}(-1)^{2(\alpha+\beta)} \frac{\left.\Gamma_{\beta}^{\alpha}\right](i t)}{\eta^{3}(i t)} A_{a b}\left[\begin{array}{l}
\alpha \\
\beta
\end{array}\right]
$$

The $\Gamma$ 's are nothing else than the derivatives of $\vartheta$-functions with respect to the variable $t$

$$
\Gamma\left[\begin{array}{l}
\alpha \\
\beta
\end{array}\right](i t)=-\frac{1}{\pi} \frac{\partial}{\partial t} \vartheta\left[\begin{array}{l}
\alpha \\
\beta
\end{array}\right](i t, 0)=\sum_{n \in \mathbb{Z}}(n+\alpha)^{2} e^{-\pi t(n+\alpha)^{2}} e^{2 \pi i(n+\alpha) \beta} .
$$

Written still differently, we have

$$
Z^{A}\left(\mathrm{E} 2_{a}, \mathrm{D} 6_{b}\right)=\int_{0}^{\infty} \frac{d t}{t} \sum_{\alpha, \beta \neq\left(\frac{1}{2}, \frac{1}{2}\right)}(-1)^{2(\alpha+\beta)} \frac{\vartheta^{\prime \prime}\left[\begin{array}{l}
\alpha \\
\beta
\end{array}\right](i t)}{\eta^{3}(i t)} A_{a b}\left[\begin{array}{l}
\alpha \\
\beta
\end{array}\right](i t)
$$

We immediately recognize the right hand side as an annulus gauge threshold correction, cf. 4.10.

Similarly, for the Möbius strip amplitude for the instanton, which as we explained we take to be invariant under the orientifold projection, it can be shown that

$$
Z^{M}\left(\mathrm{E} 2_{a}, \mathrm{O} 6\right)= \pm \int_{0}^{\infty} \frac{d t}{t} \sum_{\alpha, \beta \neq\left(\frac{1}{2}, \frac{1}{2}\right)}(-1)^{2(\alpha+\beta)} \frac{\vartheta^{\prime \prime}\left[\begin{array}{c}
\alpha \\
\beta
\end{array}\right]\left(i t+\frac{1}{2}\right)}{\eta^{3}\left(i t+\frac{1}{2}\right)} A_{a a}\left[\begin{array}{l}
\alpha \\
\beta
\end{array}\right]\left(i t+\frac{1}{2}\right)
$$

The overall plus sign is for $O(1)$ instantons, reflecting the fact that only for these the $x^{\mu}$ and $\theta^{\alpha}$ zero modes survive the orientifold projection.

In chaper 4 we saw that typically these stringy threshold corrections are non-holomorphic functions of the closed string moduli. Therefore, it is not immediately obvious in which sense the expression (5.3) is meant and how one can extract the holomorphic superpotential 
part $Y$ from it. The situation is quite similar to what we had in chapter 4 for gauge kinetic functions and gauge threshold corrections.

The CFT disk amplitudes in (5.3) are also not holomorphic but combine non-holomorphic Kähler potential contributions and holomorphic superpotential contributions in the usual way [48, 52, 2, 49] (note the similarity to $(5.2)$ ):

$$
\begin{aligned}
\left\langle\widehat{\Phi}_{a, b}[\vec{x}]\right\rangle_{\lambda_{a}, \bar{\lambda}_{b}} & =\frac{e^{\frac{\mathcal{K}}{2}} Y_{\lambda_{a} \Phi_{a, x_{1}} \Phi_{x_{1}, x_{2} \ldots \Phi_{x_{N}, b} \bar{\lambda}_{b}}}}{\sqrt{K_{\lambda_{a}, a} K_{a, x_{1}} \ldots K_{x_{n}, b} K_{b, \bar{\lambda}_{b}}}} \\
& =\frac{e^{\frac{\mathcal{K}}{2} Y_{\lambda_{a} \widehat{\Phi}_{a, b}[x] \bar{\lambda}_{b}}}}{\sqrt{K_{\lambda_{a}, a} \widehat{K}_{a, b}[x] K_{b, \bar{\lambda}_{b}}}}
\end{aligned}
$$

In chapter 4, in connection with the the Kaplunovsky-Louis formula (4.51), we saw that the stringy one-loop amplitudes include a holomorphic Wilsonian part but also nonholomorphic pieces. There we used the Kaplunovsky-Louis formula to extract gauge kinetic functions from gauge threshold corrections. Here we follow a similar strategy.

Applying the Kaplunovsky-Louis formula (4.51) to the instanton one-loop amplitudes appearing in $Z_{0}\left(\mathrm{E} 2_{a}\right)$, we write

$$
\begin{aligned}
Z_{0}\left(\mathrm{E} 2_{a}\right)=-8 & \pi^{2} \operatorname{Re}\left(f_{a}^{1-\text { loop }}\right)-\frac{b_{a}}{2} \log \left(\frac{M_{p}^{2}}{\mu^{2}}\right)-\frac{c_{a}}{2} \mathcal{K}_{\text {tree }} \\
& -\log \left(\frac{V_{3}}{g_{s}}\right)_{\text {tree }}+\sum_{b} \frac{\left|I_{a b} N_{b}\right|}{2} \log \left[\operatorname{det} K^{a b}\right]_{\text {tree }},
\end{aligned}
$$

where for the brane and instanton configuration in question the coefficients are

$$
b_{a}=\sum_{b} \frac{\left|I_{a b} N_{b}\right|}{2}-3, \quad c_{a}=\sum_{b} \frac{\left|I_{a b} N_{b}\right|}{2}-1 .
$$

The constant contributions come from the Möbius amplitude. Inserting (5.12) and (5.14) in (5.3), one sees that the (non-holomorphic) Kähler metrics involving an instanton zero mode and a matter field precisely cancel out, so that only the matter metrics survive, as required by the general form $(5.2)$. Moreover, the term $\exp (\mathcal{K} / 2)$ comes out just right due to the rule that each disk contains precisely two instanton zero modes. The holomorphic piece in 5.2 can therefore be expressed entirely in terms of other holomorphic quantities like holomorphic Yukawa couplings, the holomorphic instanton action and the one-loop holomorphic Wilsonian gauge kinetic function on the E2-instanton:

$$
\begin{aligned}
& Y_{\Phi_{a_{1}, b_{1}}, \ldots, \Phi_{a_{M}, b_{M}}}=\sum_{\text {conf. }} \operatorname{sign}_{\text {conf }} \exp \left(-S_{\mathrm{E} 2}\right)_{\text {tree }} \exp \left(-f_{a}^{1-\text { loop }}\right)
\end{aligned}
$$

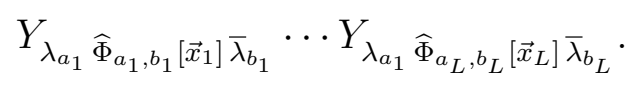


This explicitly shows that knowing the tree-level Kähler potentials, computing the matter field correlator in the instanton background up to one-loop order in $g_{s}$ is sufficient to deduce the Wilsonian holomorphic instanton generated superpotential. Higher order corrections in $g_{s}$ only come from loop corrections to the Kähler potentials.

\subsection{Corrections to Gauge Kinetic Functions}

Having discussed how to infer superpotential corrections from E2-instantons, we now consider corrections to gauge kinetic functions.

Indeed, by applying S- and T-dualities to the story of world-sheet instanton corrections in the heterotic string [66, 67, we expect that there can also be E2-instanton corrections to the holomorphic gauge kinetic functions. In the heterotic case, such corrections arise from string world-sheets of Euler characteristic zero, i.e. here from world-sheets with two boundaries. Therefore, we expect such corrections to appear for E2-instantons admitting one complex open string modulus, i.e. for those wrapping a three-cycle with Betti number $b_{1}(\Xi)=1$.

Let us discuss the instanton zero mode structure for such a cycle. First we provide the form of the vertex operators. The bosonic fields in the $(-1)$-ghost picture are

$$
V_{y}^{(-1)}(z)=y e^{-\varphi(z)} \Sigma_{h=\frac{1}{2}, q= \pm 1}(z)
$$

which, before the orientifold projection, are accompanied by the two pairs of fermionic zero modes

$$
V_{\mu}^{(-1 / 2)}(z)=\mu_{\alpha} e^{-\frac{\varphi(z)}{2}} S^{\alpha}(z) \Sigma_{h=\frac{3}{8}, q=-\frac{1}{2}}(z)
$$

and

$$
V_{\bar{\mu}}^{(-1 / 2)}(z)=\bar{\mu}_{\dot{\alpha}} e^{-\frac{\varphi(z)}{2}} S^{\dot{\alpha}}(z) \Sigma_{h=\frac{3}{8}, q=+\frac{1}{2}}(z) .
$$

Now one has to distinguish two cases depending on how the anti-holomorphic involution $\bar{\sigma}$ acts on the open string modulus $y$

$$
\bar{\sigma}: y \rightarrow \pm y .
$$

In the case that $y$ is invariant under $\bar{\sigma}$, called first kind in the following, the orientifold projection acts in the same way as for the $4 \mathrm{D}$ fields $X^{\mu}$, i.e. the two bosonic zero modes $y$ and the two fermionic zero modes $\bar{\mu}$ survive. In the other case, dubbed second kind, the bosonic zero mode is projected out and only the fermionic modulino zero mode $\mu$ survives. Therefore, in the absence of any additional zero modes, for instance from E2-D6 intersections, the zero mode measure in any instanton amplitude assumes the following forms:

$$
\int d^{4} x d^{2} \theta d^{2} y d^{2} \bar{\mu} e^{-S_{\mathrm{E} 2}} \ldots, \quad \text { for } \bar{\sigma}: y \rightarrow y
$$




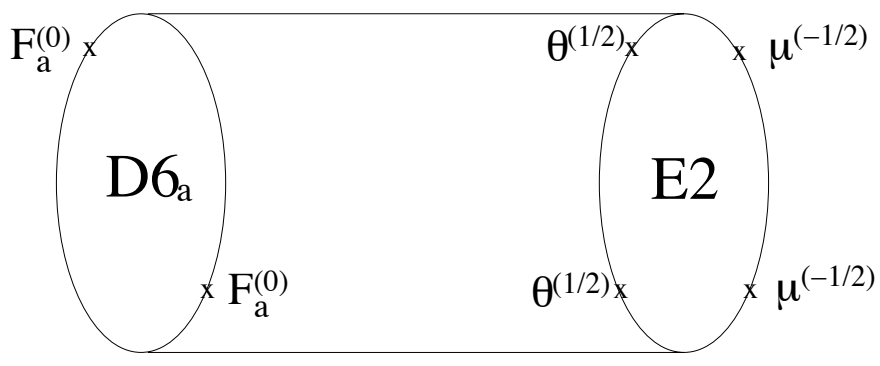

Figure 5.3: Annulus diagram for E2-instanton correction to the gauge kinetic function. The upper indices give the ghost number of the vertex operators.

and

$$
\int d^{4} x d^{2} \theta d^{2} \mu e^{-S_{\mathrm{E} 2}} \ldots, \quad \text { for } \bar{\sigma}: y \rightarrow-y
$$

Now, it is clear that an instanton with precisely one set of fermionic zero modes of the second kind and no additional zero modes can generate a correction to the $S U\left(N_{a}\right)$ gauge kinetic function. The correlator for two gauge field vertex operators in the instanton background then takes the form

$$
\left\langle F_{a}\left(p_{1}\right) F_{a}\left(p_{2}\right)\right\rangle_{\mathrm{E} 2}=\int d^{4} x d^{2} \theta d^{2} \mu \exp \left(-S_{\mathrm{E} 2}\right) \exp \left(Z_{0}^{\prime}(\mathrm{E} 2)\right) A_{F_{a}^{2}}\left(\mathrm{E} 2, D 6_{a}\right)
$$

where $A_{F_{a}^{2}}\left(\mathrm{E} 2, D 6_{a}\right)$ is the annulus diagram in figure 5.3, which absorbs all the appearing fermionic zero modes and where the gauge boson vertex operators in the (0)-ghost picture have the form

$$
V_{A}^{(0)}(z)=\epsilon^{\mu}\left(\partial_{\mu} X(z)+i(p \cdot \psi) \psi_{\mu}(z)\right) e^{i p \cdot X(z)}
$$

For further developments concerning instanton corrections to the gauge kinetic functions and an explication of how to actually perform the calculations suggested by the formulas of this section (for type I models), we refer to [38.

\subsection{Consequences for Toroidal Orbifold Models}

Let us draw some consequences for intersecting D6-brane models on toroidal orbifolds from the results of chapters 4 and 5 .

First, from the results of this chapter, there are no corrections to gauge kinetic functions for intersecting D6-brane models on $T^{6} / \mathbb{Z}_{2} \times \mathbb{Z}_{2}$, as $b_{1}(\Xi) \neq 1$ for all three-cycles $\Xi \subset$ $T^{6} / \mathbb{Z}_{2} \times \mathbb{Z}_{2}$. 
Therefore, the one-loop corrections to the gauge-kinetic functions for such models derived in chapter 4 are quantum mechanically exact, except for possible multi-instanton corrections. Second, for these models, single $O(1)$ instantons will not contribute to the superpotential, as $b_{1}(\Xi) \neq 0$, for all $\Xi$.

Thus, if we wanted to study stability questions related to single instantons, we would have to turn to models containing rigid cycles $\left(b_{1}=0\right)$ and cycles with $b_{1}=1$. One such class are models on the $T^{6} / \mathbb{Z}_{2} \times \mathbb{Z}_{2}^{\prime}$ toroidal orbifold. For this case the one-loop effects have already been worked out in [37. 
Chapter 6

Conclusions 
In this thesis we have been concerned with certain aspects of D-instantons and effective couplings such as the gauge kinetic functions and the charged matter superpotential in intersecting D6-brane models.

More specifically, after a general introduction covering the question of why to go beyond the standard model, and after giving some background of field theory instantons, in chapter 3 we showed how the non-perturbative ADS superpotential of supersymmetric QCD (SQCD) can be derived in detail as the effect of a single E2-instanton in an intersecting D6-brane setup realizing SQCD. In order to do so we first constructed such a setup and then calculated in detail the superpotential using (a slight extension of) the charged matter field instanton calculus [34], carrying out all the necessary integrations with great care. We closed with some remarks on the relation between field theory instantons and D-instantons.

Then, in chapter 4 , we looked at threshold corrections to the gauge couplings in $\mathbb{Z}_{2} \times \mathbb{Z}_{2}$ toroidal orbifold models, improving on the calculations previously found in the literature. We then went on to extract the one-loop corrections to gauge kinetic functions from our results using the field theoretic Kaplunovsky-Louis formula. By virtue of a nonrenormalization theorem, these corrections are the only perturbative corrections. In the course of the calculations it was also possible to constrain numerical constants in the Kähler metric for $\mathcal{N}=1$ chiral matter fields. Furthermore, we wrote down the universal gauge coupling corrections.

Finally, in chapter 5, we considered in greater depth the instanton calculus already used in chapter 3, showing how to extract the holomorphic superpotential in detail. The result is that knowledge of the tree level Kähler metrics is enough to infer the holomorphic Yukawa couplings from the instanton calculus. We then went on to describe which kind of instanton corrections one might expect for the gauge kinetic functions, with the result that, specializing to single $O(1)$ instantons, only instantons wrapping cycles with first Betti number $b_{1}=1$ can potentially give corrections to the gauge kinetic functions. Also, the superpotential can only receive corrections from instantons wrapping rigid cycles. As a consequence, for $\mathbb{Z}_{2} \times \mathbb{Z}_{2}$ toroidal orbifolds there are no such instanton corrections to these quantities.

We now break away slightly from the work of this thesis and describe some related ideas and how some of the material discussed here has been applied and extended. We do not intend to give a comprehensive survey, but only to present a small selection.

There are a number of topics which we have not touched upon, such as the generation of phenomenologically interesting but perturbatively forbidden couplings, as for instance Majorana mass terms, the generation of hierarchies, as well as gaugino condensation. In this connection we would like to cite [34, 87, 1, 82, 76, 33, 86, 88, 53, 13, 157].

We feel that we ought to give an impression of how some of the results discussed in this thesis have been extended or developed 11 Let us first focus on research related to the results

\footnotetext{
${ }^{1}$ Note that we certainly do not make the ridiculous claim that [7, 10, 9] were in any way 'role models'
} 
of chapter 3, i.e. to [7]. In [28] the ADS-like superpotential for gauge group $S O\left(2 N_{c}\right)$ with $N_{f}=N_{c}$ was derived in a type I setup with branes at singularities using holomorphy and various other ingredients. However, as the authors point out, the superpotential was not calculated in a full-fledged string computation of the kind presented here. In [16] instanton contributions to the superpotential and prepotential were studied in the type IIB setting with branes at singularities in an approach similar to that of chapter 3. General conditions for the generation of ADS-like superpotentials in models with D3-branes at $\mathbb{Z}_{3}$-orientifold singularities were studied in [27]. The generation of the ADS (or ADS/TVY) superpotential in locally consistent setups with magnetized D9-branes was studied in [30]. The paper [117] showed how to get non-perturbative superpotentials without introducing orientifold planes.

We now turn to papers related to chapter $4([9,7])$. Recall that in that chapter certain numerical constants in the Kähler metric for $\mathcal{N}=1$ chiral matter fields could be fixed by demanding holomorphy of the (Wilsonian) gauge kinetic functions. Similar reasoning was applied in [30] in the context of type IIB models with D9-branes. Also, in [37] a program similar to chapter 4 was carried out, i.e. gauge threshold corrections were calculated for intersecting D6-brane models on $\mathbb{Z}_{2} \times \mathbb{Z}_{2}^{\prime}$ toroidal orbifolds from which gauge kinetic functions and universal gauge coupling corrections were then extracted.

Finally, let us mention two publications related to chapter 5 ([9, 4]). In [31, 30] holomorphy issues of the instanton calculus for type IIB D-brane models were considered, very much in the spirit of our discussion. In particular, the holomorphy of the instanton induced superpotential in such setups was discussed.

Another line of research, closely related to the instanton corrections to the gauge kinetic functions considered in chapter 5, has been initiated in [38. A way to arrive at the socalled poly-instantons of that paper is the observation that there ought to be instanton corrections to the instanton corrections (and so on) to the gauge kinetic functions.

From the above (surely incomplete) discussion of research related to the topics of this thesis it easy to see that there is a lot of activity in connection with D-instantons. In particular, by now it seems to be an established principle (modeled on [7]) that new ideas for D-instanton calculations in D-brane models have to be checked against known instanton effects, such as the ADS superpotential.

Where are we headed? Even though prediction is very difficult, especially about the future (Niels Bohr) it is nevertheless possible to discern at least two possible avenues for future research. A rather obvious one would be to look at instanton corrections to the superpotential and gauge kinetic functions in models with rigid cycles and cycles with $b_{1}=1$. The simplest such models would be $\mathbb{Z}_{2} \times \mathbb{Z}_{2}^{\prime}$ toroidal orbifolds. We already remarked on this in chapter 5 and drew attention to [37] where the one-loop corrections to the gauge kinetic functions have been worked out. A detailed knowledge of instanton corrections could perhaps lead to an improved understanding of whether particular models really are stable beyond tree-level.

for the papers to be mentioned. 
Somewhat related to this is the question of whether spacetime instantons have the potential to stabilize moduli in type IIB models. One knows, or expects, this to be the case but we feel it's fair to say that a great deal remains to be understood in detail, in particular beyond single instanton effects.

Time will tell which of these ideas turn out to be fruitful. 
Appendix A

Four Dimensions of Spacetime 
When seriously considering string theory as a theory of nature, one is immediately faced with the problem that string theory requires too many dimensions of spacetime: Critical superstrings live in ten dimensions and M-theory requires one more, namely eleven. Of course, the unwanted dimensions of space can be compactified away but this is, in a way, an unjustified act of violence. It immediately provokes the question why one should leave precisely $3+1$ dimensions large $\bigsqcup^{1}$ The usual reply is that observation tells us so. This is indeed true but, as already stressed in the introduction, our aim in theoretical physics is to explain as much as we can - we hasten to add, though, that currently we cannot answer the question why spacetime is $3+1$-dimensional in a truly satisfactory way. The best understanding at current of this fact is that it is necessary on anthropic grounds: the existence of life seems to require three large dimensions of space and one large dimension of time. To our knowledge, anthropic reasons do not restrict the number of small extra dimensions.

The aim of this appendix is to summarize some of the known anthropic arguments for the dimensionality of spacetime. There do exist some genuinely 'stringy' ideas to dynamically fix the number of large dimensions of space, e.g. [40], but to our knowledge they all have their fair share of problems; for instance in the case at hand, the dimensionality of space, $d$, comes out only as $d \leq 3$.

The question about the dimensionality of space has been asked quite early on in the history of physics, even before the advent of General Relativity, notably by Immanuel Kant but surely it must have worried scientists in even earlier times. A remarkable paper on the subject by Ehrenfest, "Welche Rolle spielt die Dreidimensionalität des Raumes in den Grundgesetzen der Physik?", dates back to 1917 [73]. In this paper, Ehrenfest argued basically on anthropic grounds that space should be three-dimensional. One particular argument he gave was that in more than three dimensions of space, there are no stable planetary orbits. Let us give a freshman mechanics derivation of this.

No stable planetary orbits in $d>3$ dimensions of space. First, experimentally we only know the laws of gravity in three dimensions of space and thus some guesswork necessarily is included in extending them to higher dimensions. In the context of string theory this is no problem as we are presented with a higher dimensional theory from the outset and can infer what the higher dimensional physics will be. It is very useful in this context that gravity is one of the presumably robust predictions of string theory. In particular, at low energies we expect to get Einstein gravity and this reduces to Newtonian gravity in the weak field, low velocity limit.

If we have $d$ large dimensions of space, the gravitational potential of a point mass $M$ ('star')

\footnotetext{
${ }^{1}$ Note, incidentally, that it does not solve the problem to formulate string theory in four dimensions from the outset by replacing part of the world-sheet sigma-model by an internal CFT affording no geometric interpretation since it is obvious that this approach also does not address the question of arbitrariness.

${ }^{2}$ More precisely, he spoke of 'singular properties of $\Re_{3}$.'
} 
is

$$
\phi=-\frac{G^{(d)}}{d-2} \frac{M}{r^{d-2}} .
$$

It can be shown that the motion of a point mass $m$ ('planet') in this potential will take place in a plane containing $M$ (cf. [114]). We choose polar coordinates $r, \theta$ in that plane. Then the Lagrangian for the problem is

$$
\mathcal{L}=\frac{m}{2}\left(\dot{r}^{2}+r^{2} \dot{\theta}^{2}\right)-m \phi(r) .
$$

The variable $\theta$ is cyclic, giving us the conserved angular momentum

$$
m r^{2} \dot{\theta}=L=\text { const. }
$$

Deriving the Euler-Lagrange equation for $r$, eliminating $\dot{\theta}$, and rearranging gives

$$
\ddot{r}=-\partial_{r}\left(\frac{L}{2 m^{2} r^{2}}+\phi(r)\right)=-\partial_{r}\left(\frac{L}{2 m^{2} r^{2}}-\frac{G^{(d)}}{d-2} \frac{M}{r^{d-2}}\right) .
$$

The term in parentheses is the effective potenital. From this we can study the characteristics of the motion. A glance at the plots A.1 and A.2 convinces us that for $d \geq 5$ there are no stable orbits. For $d=4$ one has to distinguish three cases according to the value of the angular momentum. ${ }^{3}$ No surprises happen. Only the case where the angular momentum $L$ has precisely the value which makes the effective potential vanish identically merits the remark that any small perturbation, say by friction, will destroy this finetuning.

Thus, for $d>3$ dimensions of space there are no stable planetary orbits, presumably rendering life impossible. Let us mention that Ehrenfest also gave several other arguments in this direction and that an analysis of the stability of the hydrogen atom also vindicates the conclusion. A variety of arguments (lack of complexity, 'design problems' for organims, ... ) furthermore seem to indicate that for $d \leq 2$ dimensions of space life is impossible.

Therefore, the existence of life seems to require precisely three (large) dimensions of space.

Arguments for precisely one direction of time. Breaking away a little from the confines of string theory, one can also ask what would happen if time had more than one direction. We shall rely on Tegmark [138] for this question.

Even if time had more than one direction it would still be possible for an observer to perceive time as one-dimensional. This is because, if the observer is more or less pointlike,

\footnotetext{
${ }^{3}$ The angular momentum for $d=4$ can be less, equal to, or greater than the critical value which makes the effective potential vanish identically.
} 


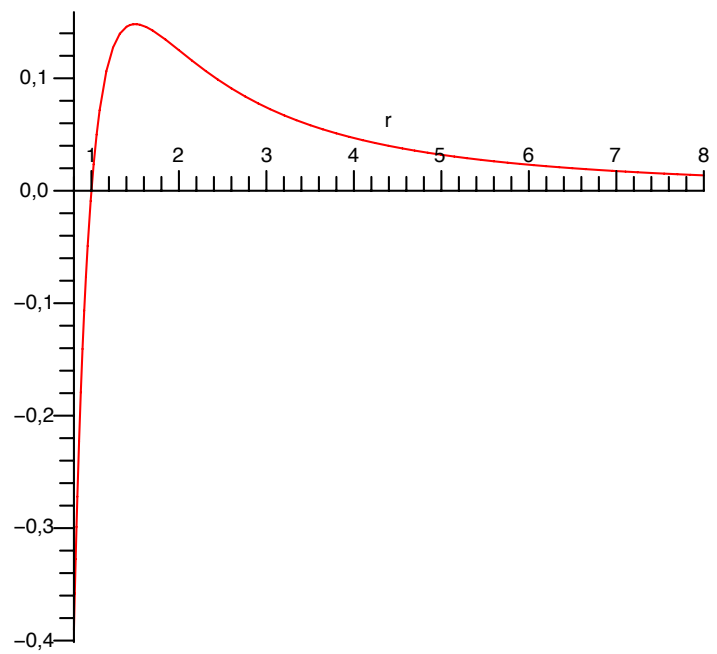

Figure A.1: Effective potential for $d=5$, arbitrary units.

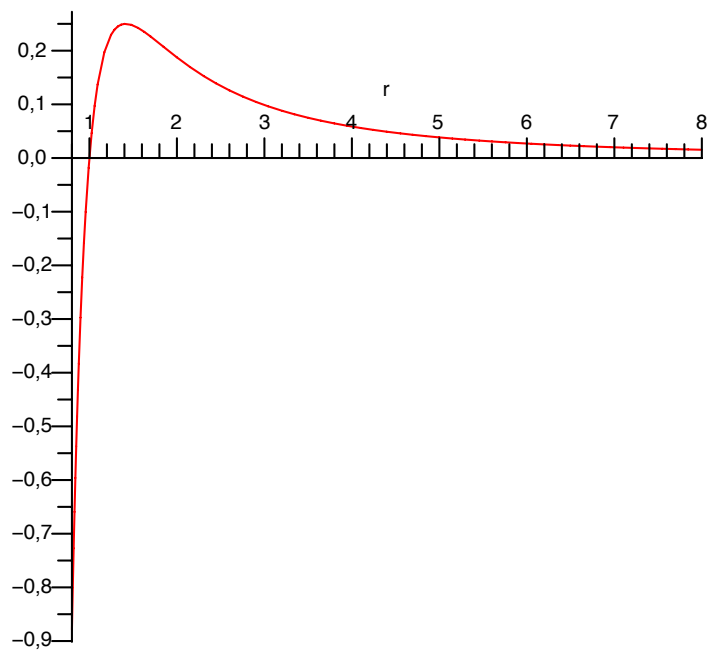

Figure A.2: Effective potential for $d=6$, arbitrary units. 


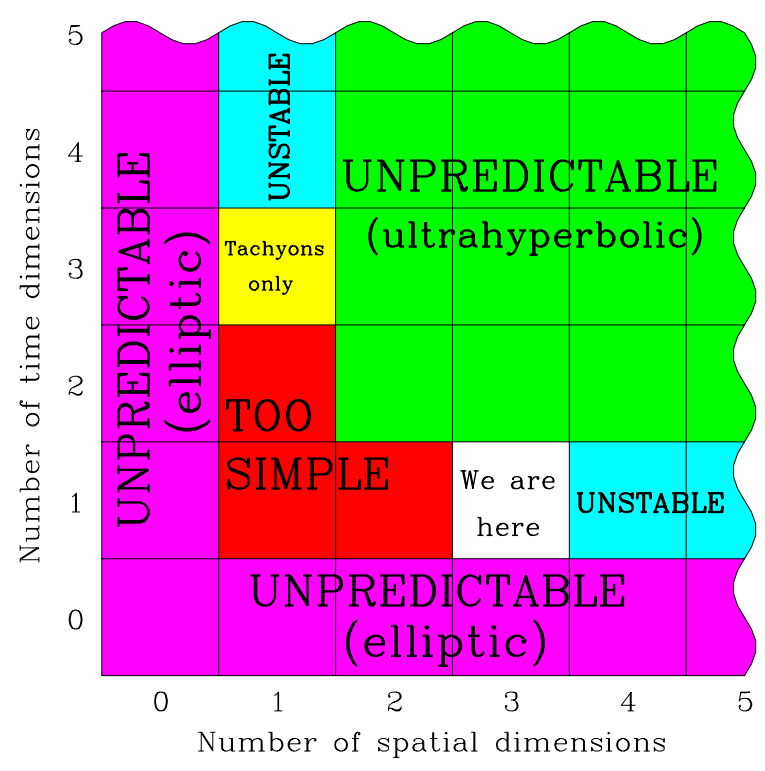

Figure A.3: Tegmark's results on why spacetime is $3+1$-dimensional. Taken from [138].

she will move on a (timelike) worldline. Since the relativity notion of proper time $(d \tau=$ $\left.\sqrt{-g_{\mu \nu} d x^{\mu} d x^{\nu}}\right)$ also applies in the case with more than one direction of time, the observer can order encountered events in linear order. Therefore, we might expect this proper time to be the notion of time our observer has. (However, such a world would be a little different than ours, as, for instance, energy becomes an $m$-vector, where $m$ is the number of time directions.)

Another interesting fact: Particles tend to become less stable when there is more than one direction of time. When there is only one time direction, a particle can only decay when its mass is greater than the sum of the masses of the particles it decays to, regardless of its kinetic energy. For more than one time direction this is no longer true. For instance,

$$
\text { proton } \rightarrow \text { neutron }+ \text { positron }+ \text { neutrino }
$$

would not a priori be ruled out for sufficiently high proton energies, since it respects all relevant quantum numbers. To reiterate: If time had more than one direction, particles would be more unstable, or only stable if they had sufficiently low energy. For the last reason, however, life could still be possible in sufficiently cold regions of that universe.

Tegmark goes on to analyze what kind of field equations are possible in a spacetime with metric of general signature and concludes that well-posed, or generally, predictive boundary problems only exist for spacetimes with signature $(-+++)$ (or the negative of this). Since, he argues, a requirement for life is being able to predict the outcomes of experiments (in the broadest sense), life requires precisely such a spacetime, i.e. three dimensions of space and one dimension of time. The situation is summarized in figure A.3. 
Comment. We have seen that there exist anthropic arguments suggesting that there should be precisely three large dimensions of space and one large dimension of time. As there are, to our knowledge, no entirely satisfactory ideas for mechanisms fixing these numbers, we have here a situation which Wilczek [147] refers to as 'Temptation.' It may well be that the observed dimensionality of spacetime simply has no deeper explanation but, in the string theory context, is only one possibility in a landscape of possibilities and we happen to observe precisely a $3+1$-dimensional spacetime because if it were different, we would not exist. However, the problem with such anthropic reasoning is that it gives no indication how to decide whether this is indeed the correct explanation and there really is no mechanism to fix the dimensionality of spacetime. 
Appendix B

Contributions of Instantons 
Any kind of perturbation theory, be it in classical or quantum physics, consists of finding a small, dimensionless parameter $\alpha$ and Taylor expanding the physical observable $A(\alpha)$ one is interested in in terms of $\alpha$. In symbols, one thus stipulates a relation of the form

$$
A(\alpha)=a_{1}+a_{2} \alpha+a_{3} \alpha^{2}+\cdots,
$$

where one has some means of calculating the $a_{\nu}$.

Now, the usefulness of such a relation depends on whether one can terminate the expansion after a few terms and obtain a good approximation to $A(\alpha)$. Quite often, say in the 'practical' applications of quantum field theory in elementary particle physics, this is indeed the case and this is the reason why there perturbation theory is a deservedly cherished tool.

However, even leaving aside questions of convergence, i.e. whether to interpret it as a power series or asymptotic expansion, there is no a priori reason why a relation such as (B.1) should hold. Indeed, suppose we have $A(0)=0$ and $A(\alpha)=e^{-1 / \alpha^{2}}$ for $\alpha \neq 0$. Then $A(\alpha)$ is infinitely often differentiable. In particular, $A(\alpha)$ has a Taylor series $T A(\alpha)=$ $\sum_{\nu} \frac{d^{\nu} A(\alpha) / d \alpha^{\nu}}{\nu !} \alpha^{\nu}$, but

$$
T A(\alpha)=0+0 \cdot \alpha+0 \cdot \alpha^{2}+\cdots \neq A(\alpha) \text { for } \alpha \neq 0 .
$$

The general, well-known lesson is that an infinitely often differentiable function can fail to be analytic. Phrased differently, our $A(\alpha)$ cannot be calculated perturbatively $\left.\right|^{1}$ and it is precisely such contributions which instantons give to physical observables.

\footnotetext{
${ }^{1}$ It is trivially clear that $A(\alpha)$ also has no useful asymptotic expansion in powers of $\alpha$. Indeed, $\mathrm{B} .2$ informs us that $A(\alpha)=\sum_{\nu=0}^{n} 0 \cdot \alpha^{\nu}+R_{n}(\alpha)$, viz. $R_{n}(\alpha)=A(\alpha)$ as $\alpha \rightarrow 0$ for all $n \geq 0$, a kind of remainder $R_{n}(\alpha)$ which can hardly be called useful.
} 
Appendix C

Euclidean Field Theory 
In this appendix we state and explain the procedures which are necessary to transfer a field theory defined in Minkowski space to the corresponding theory defined in Euclidean space 1 Since instantons are finite action solutions of Euclidean field theories, such tranfer is a necessary first step before looking for instanton solutions.

In $D$-dimensional Minkowski space the metric is $\eta_{\mu \nu}=\operatorname{diag}(1,-1, \ldots,-1) 2^{2}$ and for each contravariant vector $a^{\mu}(\mu=0,1, \ldots, D-1)$ we have the dual covector $a_{\mu}=\eta_{\mu \nu} a^{\nu}$, i.e. $a_{0}=+a^{0}, a_{1}=-a^{1}, \ldots, a_{D-1}=-a^{D-1}$.

A contravariant vector $a^{\mu}$ and a covariant vector $b_{\mu}$ can be contracted to give their Lorentzinvariant scalar product $a^{\mu} b_{\mu}$. Of particular vectors we only mention the radius vector $x^{\mu}=\left(x^{0}=t, x^{1}, \ldots, x^{D-1}\right)$ and the derivative covector $\partial_{\mu}=\left(\frac{\partial}{\partial x^{0}}, \frac{\partial}{\partial x^{1}}, \ldots, \frac{\partial}{\partial x^{D-1}}\right)$ (note the absence of minus signs here). The first of these is particularly important, since Lorentztransformations are defined as those transformations of the spacetime coordinates which leave invariant the fundamental quadratic form

$$
\left(x^{\mu} x_{\mu}\right)_{\operatorname{Min}}=+\left(x^{0}\right)^{2}-\left(x^{1}\right)^{2}-\cdots-\left(x^{D-1}\right)^{2} .
$$

In $D$-dimensional Euclidean space the metric is $\delta_{\mu \nu}=\operatorname{diag}(1, \ldots, 1)$ leading to the situation that we do not have to distinguish between co- and contravariant vectors, since $a_{\mu}=\delta_{\mu \nu} a^{\nu}$ gives $a_{\mu}=a^{\mu}$ for $\mu=1, \ldots, D$. In particular

$$
\left(x^{\mu} x_{\mu}\right)_{\mathrm{Euc}}=\left(x^{\mu} x^{\mu}\right)_{\mathrm{Euc}}=\left(x^{1}\right)^{2}+\cdots+\left(x^{D}\right)^{2},
$$

which is invariant precisely under $O(D)$ transformations of the coordinates $x^{\mu}$.

Now, passing from the Minkowskian version of a field theory to its Euclidean counterpart simply amounts to replacing any Minkowki vector (and tensor) by its Euclidean equivalent. Note that the Euclidean theory is a completely different theory because Euclidean $O(D)$ rotations are quite different from Lorentz transformations. However, there is a formal device which makes it possible to think of the Euclidean theory as a kind of analytic continuation of the theory in Minkowski space. This consists of taking the time coordinate $x^{0}=t$ of the Minkowskian theory and formally continuing it to imaginary values, whereafter $\left(x^{D}\right)_{\text {Euc }}=i t$ can serve as the real $D$-th coordinate, while all other coordinates remain unchanged.

After this, we have

$$
\left(x^{\mu} x^{\mu}\right)_{\mathrm{Euc}}=-\left(x^{\mu} x_{\mu}\right)_{\mathrm{Min}}
$$

(note the minus sign), and a similar procedure has to be applied to any Lorentz vector and tensor.

To obtain the Euclidean action $S_{\text {Euc }}$ from the Minkowskian $S_{\text {Min }}$, we perform the procedure just outlined on $S_{\text {Min }}$ and afterwards multiply it by $-i$, so that

$$
S_{\text {Euc }}=-i\left(S_{\text {Min }}\right)_{x^{0}=i t \text { etc. }} .
$$

\footnotetext{
${ }^{1}$ We have borrowed extensively from 123 for the preparation of this section.

${ }^{2}$ For field theory we mostly use the mostly minus convention.
} 
The multiplication by $-i$ is convenient in the quantum theory.

Let us illustrate this procedure first on a simple example. Consider the Klein-Gordon system in Minkowski space with action

$$
S_{\text {Min }}=\int d x^{0} \int d x^{1}\left[\frac{1}{2}\left(\frac{\partial \phi}{\partial x^{0}}\right)^{2}-\frac{1}{2}\left(\frac{\partial \phi}{\partial x^{1}}\right)^{2}-m^{2} \phi\right] .
$$

The field equation derived from this action is

$$
\left[\left(\frac{\partial}{\partial x^{0}}\right)^{2}-\left(\frac{\partial}{\partial x^{1}}\right)^{2}\right] \phi+m^{2} \phi=0 \quad \text { (Minkowskian) }
$$

If we carry out the replacement $\left(\partial^{\mu} \partial_{\mu}\right)_{\text {Min }}=-\left(\partial^{\mu} \partial^{\mu}\right)_{\text {Euc }}$ in the Minkowskian field equation (C.6), we get the Euclidean field equation

$$
\left.-\left[\left(\frac{\partial}{\partial x^{2}}\right)^{2}+\left(\frac{\partial}{\partial x^{1}}\right)^{2}\right] \phi+m^{2} \phi=0 \quad \text { (Euclidean }\right) .
$$

If we carry out the procedure outlined above for the Minkowskian action (C.5), we obtain the Euclidean action

$$
S_{\mathrm{Euc}}=\int d x^{2} \int d x^{1}\left[\frac{1}{2}\left(\frac{\partial \phi}{\partial x^{2}}\right)^{2}+\frac{1}{2}\left(\frac{\partial \phi}{\partial x^{1}}\right)^{2}+m^{2} \phi\right],
$$

from which follows the same field equation as in (C.7).

Note that we can also go from the Euclidean theory to the Minkowskian theory by putting $x^{D}=i x^{0}$ etc.

As another example we take pure Yang-Mills theory with semi-simple gauge group $G$, at first in Minkowski space. Choose some representation $T^{a}(a=1, \ldots, N)$ of (the Lie algebra of) $G$.

For example, if $G$ were $S U(2)$ and we chose the fundamental (=isospinor) representation of $S U(2)$ we then would have $T^{a}=-i \sigma^{a} / 2$ with

$$
\sigma^{1}=\left(\begin{array}{cc}
0 & 1 \\
1 & 0
\end{array}\right), \quad \sigma^{2}=\left(\begin{array}{cc}
0 & -i \\
i & 0
\end{array}\right), \quad \sigma^{3}=\left(\begin{array}{cc}
1 & 0 \\
0 & -1
\end{array}\right)
$$

the familiar Pauli-matrices.

The basic dynamical variables in the theory are the gauge fields $A_{\mu}^{a}(x)(a=1, \ldots, N)$, but it is convenient to also define a single matrix valued gauge field

$$
A_{\mu}(x)=g \sum_{a} T^{a} A_{\mu}^{a}(x),
$$


with $g$ the coupling constant.

Gauge transformations

$$
U(x)=\exp \left(\theta^{a}(x) T^{a}\right)
$$

act on the gauge field, respectively the field strength, as

$$
A_{\mu} \rightarrow U A_{\mu} U^{-1}+U \partial_{\mu} U^{-1}
$$

and

$$
F_{\mu \nu} \rightarrow U F_{\mu \nu} U^{-1}
$$

The gauge invariant Minkowskian action is

$$
S_{\mathrm{Min}}=\frac{1}{2 g^{2}} \int d^{4} x \operatorname{tr} F_{\mu \nu} F^{\mu \nu} .
$$

Applying the procedure for continuation to Euclidean space in this case, i.e. continuing $x^{0}, A_{0}(x)$ etc. to imaginary values and multiplying the action by $-i$ gives the Euclidean action (see, e.g. [140])

$$
S_{\mathrm{Euc}}=-\frac{1}{2 g^{2}} \int d^{4} x \operatorname{tr} F_{\mu \nu} F_{\mu \nu},
$$

from which we get the Euclidean Yang-Mills equations

$$
D_{\lambda} F_{\mu \nu} \equiv \partial_{\lambda} F_{\mu \nu}+\left[A_{\lambda}, F_{\mu \nu}\right]=0 \quad \text { (Euclidean YM) }
$$


Appendix D

Some Relations in Linear Algebra 
In this appendix we gather some results in linear algebra necessary for the calculations in chapter 3. They are all well known but it might be helpful to have them in one place.

\section{General formulas}

- The derivative of the determinant of a matrix $A$ with respect to the matrix element $A_{i, j}$ is (Jacobi)

$$
\frac{\partial}{\partial A_{i, j}} \operatorname{det} A=\operatorname{det} A \cdot\left(A^{-1}\right)_{j, i}
$$

- The determinant of a block-matrix with mutually commuting matrices $A, B, C, D$ can be computed using the formula

$$
\begin{aligned}
& {\left[\begin{array}{ll}
A & B \\
C & D
\end{array}\right]^{-1}} \\
& \quad=\left[\begin{array}{cc}
A^{-1}+A^{-1} B\left(D-C A^{-1} B\right)^{-1} C A^{-1} & -A^{-1} B\left(D-C A^{-1} B\right)^{-1} \\
-\left(D-C A^{-1} B\right)^{-1} C A^{-1} & \left(D-C A^{-1} B\right)^{-1}
\end{array}\right] .
\end{aligned}
$$

- If the matrices $A, B, C, D$ mutually commute, the following holds

$$
\operatorname{det}\left[\begin{array}{ll}
A & B \\
C & D
\end{array}\right]=\operatorname{det}(A D-B C) \text {. }
$$

- For an $N \times N$ matrix the following relation holds, where, as in chapter $3,\left.A\right|_{k, i}$ denotes the matrix $A$ with row $k$ and column $i$ deleted

$$
\operatorname{det} A \delta_{i, j}=\left.\sum_{k=1}^{N}(-1)^{i+k} A_{k, j} \operatorname{det} A\right|_{k, i} .
$$

- For an $N \times(N-1)$ matrix $A$ and an $(N-1) \times N$ matrix $B$ it is clear that the determinant of the $N \times N$ matrix $A B$ vanishes:

$$
\operatorname{det} A B=0 .
$$

- The $(i, j)$ th element of the inverse matrix $A^{-1}$ is

$$
A_{i, j}^{-1}=(-1)^{i+j} \frac{\left.\operatorname{det} A\right|_{j, i}}{\operatorname{det} A} .
$$

- For $N \times N$ matrices $A$ and $B$ we have

$$
\left.\left.\sum_{k} \operatorname{det} A\right|_{i, k} \cdot \operatorname{det} B\right|_{k, j}=\left.\operatorname{det} A B\right|_{i, j} .
$$




\section{A determinant formula}

Let $A$ be an $N \times(N-1)$ matrix and $B$ an $(N-1) \times N$ matrix.

Define

$$
\widetilde{A}=\left[A_{N,(N-1)}, 0_{N, 1}\right] \quad \text { and } \quad \widetilde{B}=\left[\begin{array}{c}
B_{(N-1), N} \\
0_{1, N}
\end{array}\right],
$$

and note that $A B=\widetilde{A} \widetilde{B}$.

Then one finds using (D.7)

$$
\begin{aligned}
& \left.\sum_{i=1}^{N} \operatorname{det}\left(A B\left((A B+\alpha \mathbb{1})^{2}+\beta^{2} \mathbb{1}\right)\right)\right|_{i, i} \\
= & \left.\sum_{i=1}^{N} \operatorname{det}\left(A B\left((A B)^{2}+2 \alpha A B+\alpha^{2} \mathbb{1}+\beta^{2} \mathbb{1}\right)\right)\right|_{i, i} \\
= & \left.\left.\sum_{i, j=1}^{N} \operatorname{det} A B\right|_{i, j} \operatorname{det}\left((A B)^{2}+2 \alpha A B+\alpha^{2} \mathbb{1}+\beta^{2} \mathbb{1}\right)\right|_{j, i} \\
= & \left.\left.\sum_{i, j=1}^{N} \operatorname{det} \widetilde{A} \widetilde{B}\right|_{i, j} \operatorname{det}\left((\widetilde{A} \widetilde{B})^{2}+2 \alpha \widetilde{A} \widetilde{B}+\alpha^{2} \mathbb{1}+\beta^{2} \mathbb{1}\right)\right|_{j, i} \\
= & \left.\left.\left.\sum_{i, j, k=1}^{N} \operatorname{det} \widetilde{A}\right|_{i, k} \operatorname{det} \widetilde{B}\right|_{k, j} \operatorname{det}\left((\widetilde{A} \widetilde{B})^{2}+2 \alpha \widetilde{A} \widetilde{B}+\alpha^{2} \mathbb{1}+\beta^{2} \mathbb{1}\right)\right|_{j, i} .
\end{aligned}
$$

Now note that $\left.\operatorname{det} \widetilde{A}\right|_{i, k}$ and $\left.\operatorname{det} \widetilde{B}\right|_{k, j}$ are only nonzero for $k=N$. Therefore, one can write

$$
\begin{aligned}
& \left.\left.\left.\sum_{i, j=1}^{N} \operatorname{det} \widetilde{B}\right|_{N, j} \operatorname{det}\left((\widetilde{A} \widetilde{B})^{2}+2 \alpha \widetilde{A} \widetilde{B}+\alpha^{2} \mathbb{1}+\beta^{2} \mathbb{1}\right)\right|_{j, i} \operatorname{det} \widetilde{A}\right|_{i, N} \\
= & \left.\operatorname{det}\left(\widetilde{B}\left((\widetilde{A} \widetilde{B})^{2}+2 \alpha \widetilde{A} \widetilde{B}+\alpha^{2} \mathbb{1}+\beta^{2} \mathbb{1}\right) \widetilde{A}\right)\right|_{N, N} \\
= & \left.\operatorname{det}\left((\widetilde{B} \widetilde{A})^{3}+2 \alpha(\widetilde{B} \widetilde{A})^{2}+\left(\alpha^{2}+\beta^{2}\right) \widetilde{B} \widetilde{A}\right)\right|_{N, N} .
\end{aligned}
$$

Finally, observe that

$$
\widetilde{B} \widetilde{A}=\left[\begin{array}{cc}
B A & 0 \\
0 & 0
\end{array}\right]
$$

which then implies that (D.10) is equal to

$$
\begin{aligned}
& \operatorname{det}\left((B A)^{3}+2 \alpha(B A)^{2}+\left(\alpha^{2}+\beta^{2}\right) B A\right) \\
= & \operatorname{det}\left(B A\left((B A+\alpha \mathbb{1})^{2}+\beta^{2} \mathbb{1}\right)\right) .
\end{aligned}
$$


This computation shows that (D.9) is equal to (D.11).

\section{A formula concerning characteristic polynomials}

Let $A$ be an $N \times(N-k)$ matrix and $B$ an $(N-k) \times N$ matrix for $k=1, \ldots,(N-1)$.

Then define

$$
\widetilde{A}=\left[A_{N,(N-k)}, 0_{N, k}\right] \quad \text { and } \quad \widetilde{B}=\left[\begin{array}{c}
B_{(N-k), N} \\
0_{k, N}
\end{array}\right]
$$

and note that $A B=\widetilde{A} \widetilde{B}$. Further, denote the characteristic polynomial of a matrix $M$ as $\chi_{M}(\sigma)$ and recall that $\chi_{M N}(\sigma)=\chi_{N M}(\sigma)$ for square matrices $M, N$.

Then it is easy to see that

$$
\begin{aligned}
\operatorname{det}[A B+\lambda \mathbb{1}] & =\operatorname{det}[\widetilde{A} \widetilde{B}+\lambda \mathbb{1}] \\
& =\chi_{\widetilde{A} \widetilde{B}}(-\lambda) \\
& =\chi_{\widetilde{B} \widetilde{A}}(-\lambda) \\
& =\operatorname{det}[\widetilde{B} \widetilde{A}+\lambda \mathbb{1}] \\
& =\operatorname{det}\left[\begin{array}{llll}
B A+\lambda & & & \\
& \lambda & & \\
& & \ddots & \\
& & & \lambda
\end{array}\right] \\
& =\lambda^{k} \operatorname{det}[B A+\lambda \mathbb{1}] .
\end{aligned}
$$




\section{Acknowledgements}

There are a great many people and institutions to whom I am very much indebted and it is a pleasure to express my gratitude towards them here.

First of all, I want to thank PD Dr. Ralph Blumenhagen for supervising this thesis and thus allowing me to learn from him how a practicing string theorist approaches the subject. I am enormously indebted to him for all the support he gave, including all the times he pulled me through the difficult and frustrating parts of research.

Likewise, I thank Prof. Dr. Dieter Lüst for agreeing to serve as the second referee to this thesis and for his constant and crucial support and encouragement. He too deserves a fair share of the credit for introducing me to string theory. Also, by far-sight and hard work he has created a very stimulating scientific environment in the string theory groups at the Max-Planck-Institute for Physics and the University of Munich from which I could benefit.

I have also drawn up a list of people from all of whom I have learned a lot of physics and who all have helped me in one way or another during my PhD studies (I apologize in advance for inadvertently leaving anyone out). They are, in alphabetical order: Hendrik Adorf, Murad Alim, Otmar Biebel, Gabriel Lopes Cardoso, Michael Flohr, Florian Gmeiner, Carsten Grabow, Michael Haack, Robert Helling, Philipp Höffer von Loewenfeld, Stefan Höhne, Matthias Kaminski, Michael Köhn, Sebastian Moster, Jan Perz, Erik Plauschinn, Michael Plümacher, Robert Richter, Christian Römelsberger, Felix Rust, Christian Sämann, Jochen Schieck, Maximilian Schmidt-Sommerfeld, Frank Steffen, Johannes Oberreuter, Timo Weigand, and Marco Zagermann. Thank you!

Two names from the preceding list rightly deserve to be mentioned again separately because I owe such an inordinate amount of physics insights and deep discussions to them. They are (again in alphabetical order): Christian Sämann and Max Schmidt-Sommerfeld.

Collaboration with Ralph Blumenhagen, Dieter Lüst, Maximilian Schmidt-Sommerfeld and Erik Plauschinn is gratefully acknowledged.

I extend my thanks to the Max-Planck-Institute for Physics and the IMPRS program of the Max-Planck-Society for financial support. Also, thanks are due to the current, respectively the former coordinator of the IMPRS for Particle Physics, Frank Steffen and Jochen Schieck, as well as to the secretaries Franziska Rudert (IMPRS), Rosita Jurgeleit, 
and Carola Reinke (both of the theory group at the MPI for Physics).

An undertaking such as a $\mathrm{PhD}$ is greatly alleviated if one can rely on friends and family and thus I warmly thank my parents and grandparents as well as the Paul and Scheibenzuber families for continued support.

Finally, I thank my beloved wife Heike: I would not have made it without you! 


\section{Bibliography}

[1] S. A. Abel and M. D. Goodsell. Realistic Yukawa couplings through instantons in intersecting brane worlds. JHEP, 10:034, 2007, hep-th/0612110.

[2] S. A. Abel and A. W. Owen. Interactions in intersecting brane models. Nucl. Phys., B663:197-214, 2003, hep-th/0303124.

[3] I. Affleck. On constrained instantons. Nucl. Phys., B191:429, 1981.

[4] I. Affleck, M. Dine, and N. Seiberg. Supersymmetry Breaking by Instantons. Phys. Rev. Lett., 51:1026, 1983.

[5] I. Affleck, M. Dine, and N. Seiberg. Dynamical Supersymmetry Breaking in Supersymmetric QCD. Nucl. Phys., B241:493-534, 1984.

[6] O. Aharony, S. S. Gubser, J. M. Maldacena, H. Ooguri, and Y. Oz. Large N field theories, string theory and gravity. Phys. Rept., 323:183-386, 2000, hep-th/9905111.

[7] N. Akerblom, R. Blumenhagen, D. Lüst, E. Plauschinn, and M. Schmidt-Sommerfeld. Non-perturbative sqcd superpotentials from string instantons. JHEP, 04:076, 2007, hep-th/0612132.

[8] N. Akerblom, R. Blumenhagen, D. Lüst, and M. Schmidt-Sommerfeld. D-brane Instantons in 4D Supersymmetric String Vacua. 2007, arXiv:0712.1793 [hep-th].

[9] N. Akerblom, R. Blumenhagen, D. Lüst, and M. Schmidt-Sommerfeld. Instantons and Holomorphic Couplings in Intersecting D- brane Models. JHEP, 08:044, 2007, arXiv:0705.2366 [hep-th].

[10] N. Akerblom, R. Blumenhagen, D. Lüst, and M. Schmidt-Sommerfeld. Thresholds for intersecting D-branes revisited. Phys. Lett., B652:53-59, 2007, arXiv:0705.2150 [hep-th].

[11] I. Antoniadis, C. Bachas, and E. Dudas. Gauge couplings in four-dimensional type i string orbifolds. Nucl. Phys., B560:93-134, 1999, hep-th/9906039.

[12] I. Antoniadis, C. Bachas, C. Fabre, H. Partouche, and T. R. Taylor. Aspects of type I - type II - heterotic triality in four dimensions. Nucl. Phys., B489:160-178, 1997, hep-th/9608012. 
[13] S. Antusch, L. E. Ibanez, and T. Macri. Neutrino Masses and Mixings from String Theory Instantons. JHEP, 09:087, 2007, 0706.2132.

[14] T. Aoyama, M. Hayakawa, T. Kinoshita, and M. Nio. Revised value of the eighthorder electron g-2. Phys. Rev. Lett., 99:110406, 2007, arXiv:0706.3496 [hep-ph].

[15] R. Argurio, M. Bertolini, G. Ferretti, A. Lerda, and C. Petersson. Stringy Instantons at Orbifold Singularities. JHEP, 06:067, 2007, 0704.0262.

[16] R. Argurio, M. Bertolini, G. Ferretti, A. Lerda, and C. Petersson. Stringy Instantons at Orbifold Singularities. JHEP, 06:067, 2007, 0704.0262.

[17] R. Argurio, M. Bertolini, S. Franco, and S. Kachru. Metastable vacua and D-branes at the conifold. JHEP, 06:017, 2007, hep-th/0703236.

[18] M. F. Atiyah, N. J. Hitchin, V. G. Drinfeld, and Y. I. Manin. Construction of instantons. Phys. Lett., A65:185-187, 1978.

[19] C. Bachas and C. Fabre. Threshold Effects in Open-String Theory. Nucl. Phys., B476:418-436, 1996, hep-th/9605028.

[20] C. Beasley and E. Witten. Residues and world-sheet instantons. JHEP, 10:065, 2003, hep-th/0304115.

[21] C. Beasley and E. Witten. New instanton effects in string theory. JHEP, 02:060, 2006, hep-th/0512039.

[22] K. Becker, M. Becker, and J. H. Schwarz. String theory and M-theory: A modern introduction. Cambridge, UK: Cambridge Univ. Pr. (2007) 739 p.

[23] K. Becker, M. Becker, and A. Strominger. Five-branes, membranes and nonperturbative string theory. Nucl. Phys., B456:130-152, 1995, hep-th/9507158.

[24] A. A. Belavin, A. M. Polyakov, A. S. Shvarts, and Y. S. Tyupkin. Pseudoparticle solutions of the Yang-Mills equations. Phys. Lett., B59:85-87, 1975.

[25] M. Berg, M. Haack, and B. Kors. Loop corrections to volume moduli and inflation in string theory. Phys. Rev., D71:026005, 2005, hep-th/0404087.

[26] M. Bertolini, M. Billo, A. Lerda, J. F. Morales, and R. Russo. Brane world effective actions for d-branes with fluxes. Nucl. Phys., B743:1-40, 2006, hep-th/0512067.

[27] M. Bianchi, F. Fucito, and J. F. Morales. D-brane instantons on the t6/z3 orientifold. 2007, arXiv:0704.0784 [hep-th].

[28] M. Bianchi and E. Kiritsis. Non-perturbative and flux superpotentials for type i strings on the z3 orbifold. 2007, hep-th/0702015.

[29] M. Billo et al. Classical gauge instantons from open strings. JHEP, 02:045, 2003, hep-th/0211250. 
[30] M. Billo et al. Instanton effects in $\mathrm{N}=1$ brane models and the Kahler metric of twisted matter. JHEP, 12:051, 2007, 0709.0245.

[31] M. Billo et al. Instantons in N=2 magnetized D-brane worlds. JHEP, 10:091, 2007, 0708.3806.

[32] R. Blumenhagen, M. Cvetic, P. Langacker, and G. Shiu. Toward realistic intersecting D-brane models. Ann. Rev. Nucl. Part. Sci., 55:71-139, 2005, hep-th/0502005.

[33] R. Blumenhagen, M. Cvetic, D. Lüst, R. Richter, and T. Weigand. Non-perturbative Yukawa Couplings from String Instantons. Phys. Rev. Lett., 100:061602, 2008, 0707.1871.

[34] R. Blumenhagen, M. Cvetic, and T. Weigand. Spacetime instanton corrections in 4d string vacua - the seesaw mechanism for d-brane models. Nucl. Phys., B771:113-142, 2007, hep-th/0609191.

[35] R. Blumenhagen, B. Körs, D. Lüst, and S. Stieberger. Four-dimensional String Compactifications with D-Branes, Orientifolds and Fluxes. Phys. Rept., 445:1-193, 2007, hep-th/0610327.

[36] R. Blumenhagen, D. Lüst, and S. Stieberger. Gauge unification in supersymmetric intersecting brane worlds. JHEP, 07:036, 2003, hep-th/0305146.

[37] R. Blumenhagen and M. Schmidt-Sommerfeld. Gauge Thresholds and Kaehler Metrics for Rigid Intersecting D-brane Models. JHEP, 12:072, 2007, arXiv:0711.0866 [hep-th].

[38] R. Blumenhagen and M. Schmidt-Sommerfeld. Power Towers of String Instantons for $\mathrm{N}=1$ Vacua. 2008, 0803.1562.

[39] R. Bousso and J. Polchinski. Quantization of four-form fluxes and dynamical neutralization of the cosmological constant. JHEP, 06:006, 2000, hep-th/0004134.

[40] R. H. Brandenberger and C. Vafa. Superstrings in the Early Universe. Nucl. Phys., B316:391, 1989.

[41] E. I. Buchbinder, R. Donagi, and B. A. Ovrut. Superpotentials for vector bundle moduli. Nucl. Phys., B653:400-420, 2003, hep-th/0205190.

[42] J. Callan, Curtis G., R. F. Dashen, and D. J. Gross. A Mechanism for Quark Confinement. Phys. Lett., B66:375, 1977.

[43] S. M. Carroll. The cosmological constant. Living Rev. Rel., 4:1, 2001, astro$\mathrm{ph} / 0004075$.

[44] T. P. Cheng and L. F. Li. Gauge Theory of Elementary Particle Physics. Oxford, Uk: Clarendon (1984) 536 p. (Oxford Science Publications). 
[45] G. A. Christos. Some Aspects of the U(1) Problem and the Pseudoscalar Mass Spectrum. Austral. J. Phys., 37:241-254, 1984.

[46] S. R. Coleman. The uses of instantons. Subnucl. Ser., 15:805, 1979.

[47] S. F. Cordes. The Instanton Induced Superpotential in Supersymmetric QCD. Nucl. Phys., B273:629, 1986.

[48] D. Cremades, L. E. Ibanez, and F. Marchesano. Yukawa couplings in intersecting d-brane models. JHEP, 07:038, 2003, hep-th/0302105.

[49] D. Cremades, L. E. Ibanez, and F. Marchesano. Computing yukawa couplings from magnetized extra dimensions. JHEP, 05:079, 2004, hep-th/0404229.

[50] R. J. Crewther. Chirality Selection Rules and the U(1) Problem. Phys. Lett., B70:349, 1977.

[51] R. J. Crewther, P. Di Vecchia, G. Veneziano, and E. Witten. Chiral Estimate of the Electric Dipole Moment of the Neutron in Quantum Chromodynamics. Phys. Lett., B88:123, 1979.

[52] M. Cvetic and I. Papadimitriou. Conformal field theory couplings for intersecting d-branes on orientifolds. Phys. Rev., D68:046001, 2003, hep-th/0303083.

[53] M. Cvetic, R. Richter, and T. Weigand. Computation of d-brane instanton induced superpotential couplings: Majorana masses from string theory. 2007, hepth/0703028.

[54] M. Cvetic, R. Richter, and T. Weigand. (Non-)BPS bound states and D-brane instantons. 2008, arXiv:0803.2513 [hep-th].

[55] M. Cvetic, G. Shiu, and A. M. Uranga. Chiral four-dimensional N = 1 supersymmetric type IIA orientifolds from intersecting D6-branes. Nucl. Phys., B615:3-32, 2001, hep-th/0107166.

[56] M. Cvetic, G. Shiu, and A. M. Uranga. Three-family supersymmetric standard like models from intersecting brane worlds. Phys. Rev. Lett., 87:201801, 2001, hepth/0107143.

[57] M. Cvetic and T. Weigand. Hierarchies from D-brane instantons in globally defined Calabi-Yau Orientifolds. 2007, 0711.0209.

[58] C. T. H. Davies et al. High-precision lattice QCD confronts experiment. Phys. Rev. Lett., 92:022001, 2004, hep-lat/0304004.

[59] A. C. Davis, M. Dine, and N. Seiberg. The Massless Limit of Supersymmetric QCD. Phys. Lett., B125:487, 1983. 
[60] J.-P. Derendinger, S. Ferrara, C. Kounnas, and F. Zwirner. All loop gauge couplings from anomaly cancellation in string effective theories. Phys. Lett., B271:307-313, 1991.

[61] J. P. Derendinger, S. Ferrara, C. Kounnas, and F. Zwirner. On loop corrections to string effective field theories: Field dependent gauge couplings and sigma model anomalies. Nucl. Phys., B372:145-188, 1992.

[62] B. S. DeWitt. Quantum Theory of Gravity. 1. The Canonical Theory. Phys. Rev., 160:1113-1148, 1967.

[63] B. S. DeWitt. Quantum theory of gravity. II. The manifestly covariant theory. Phys. Rev., 162:1195-1239, 1967.

[64] B. S. DeWitt. Quantum theory of gravity. III. Applications of the covariant theory. Phys. Rev., 162:1239-1256, 1967.

[65] M. Dine. Supersymmetry and string theory: Beyond the standard model. Cambridge, UK: Cambridge Univ. Pr. (2007) 515 p.

[66] M. Dine, N. Seiberg, X. G. Wen, and E. Witten. Nonperturbative Effects on the String World Sheet. Nucl. Phys., B278:769, 1986.

[67] M. Dine, N. Seiberg, X. G. Wen, and E. Witten. Nonperturbative Effects on the String World Sheet. 2. Nucl. Phys., B289:319, 1987.

[68] J. F. Donoghue. General relativity as an effective field theory: The leading quantum corrections. Phys. Rev., D50:3874-3888, 1994, gr-qc/9405057.

[69] N. Dorey, T. J. Hollowood, and V. V. Khoze. A brief history of the stringy instanton. 2000, hep-th/0010015.

[70] N. Dorey, T. J. Hollowood, V. V. Khoze, and M. P. Mattis. The calculus of many instantons. Phys. Rept., 371:231-459, 2002, hep-th/0206063.

[71] M. R. Douglas. Branes within branes. 1995, hep-th/9512077.

[72] M. R. Douglas. Gauge Fields and D-branes. J. Geom. Phys., 28:255-262, 1998, hep-th/9604198.

[73] P. Ehrenfest. Welche Rolle spielt die Dreidimensionalität des Raumes in den Grundgesetzen der Physik? Annalen der Physik, 366:440-446, 1920.

[74] R. P. Feynman. Quantum theory of gravitation. Acta Phys. Polon., 24:697-722, 1963.

[75] D. Finnell and P. Pouliot. Instanton calculations versus exact results in four- dimensional SUSY gauge theories. Nucl. Phys., B453:225-239, 1995, hep-th/9503115.

[76] B. Florea, S. Kachru, J. McGreevy, and N. Saulina. Stringy instantons and quiver gauge theories. JHEP, 05:024, 2007, hep-th/0610003. 
[77] S. Förste, G. Honecker, and R. Schreyer. Supersymmetric Z(N) x Z(M) orientifolds in 4D with D- branes at angles. Nucl. Phys., B593:127-154, 2001, hep-th/0008250.

[78] O. J. Ganor. A note on zeroes of superpotentials in F-theory. Nucl. Phys., B499:5566, 1997, hep-th/9612077.

[79] M. B. Green and M. Gutperle. Effects of D-instantons. Nucl. Phys., B498:195-227, 1997, hep-th/9701093.

[80] M. B. Green, J. H. Schwarz, and E. Witten. Superstring Theory, vol. 1: Introduction. Cambridge, UK: Univ. Pr. (1987) 469 p. (Cambridge Monographs On Mathematical Physics).

[81] M. B. Green, J. H. Schwarz, and E. Witten. Superstring Theory, vol. 2: Loop Amplitudes, Anomalies and Phenomenology. Cambridge, UK: Univ. Pr. (1987) 596 p. (Cambridge Monographs On Mathematical Physics).

[82] M. Haack, D. Krefl, D. Lüst, A. Van Proeyen, and M. Zagermann. Gaugino condensates and D-terms from D7-branes. JHEP, 01:078, 2007, hep-th/0609211.

[83] J. A. Harvey and G. W. Moore. Superpotentials and membrane instantons. 1999, hep-th/9907026.

[84] E. Hobson. The Theory of Functions of a Real Variable and the Theory of Fourier's Series, Vol. 2. New York: Dover Publications (1957) 2nd edition.

[85] L. E. Ibanez and D. Lüst. Duality anomaly cancellation, minimal string unification and the effective low-energy lagrangian of 4-d strings. Nucl. Phys., B382:305-364, 1992, hep-th/9202046.

[86] L. E. Ibanez, A. N. Schellekens, and A. M. Uranga. Instanton Induced Neutrino Majorana Masses in CFT Orientifolds with MSSM-like spectra. JHEP, 06:011, 2007, arXiv:0704.1079 [hep-th].

[87] L. E. Ibanez and A. M. Uranga. Neutrino majorana masses from string theory instanton effects. JHEP, 03:052, 2007, hep-th/0609213.

[88] L. E. Ibanez and A. M. Uranga. Instanton Induced Open String Superpotentials and Branes at Singularities. JHEP, 02:103, 2008, 0711.1316.

[89] C. J. Isham. Prima facie questions in quantum gravity. 1993, gr-qc/9310031.

[90] C. V. Johnson. D-branes. Cambridge, USA: Univ. Pr. (2003) 548 p.

[91] S. Kachru, R. Kallosh, A. Linde, and S. P. Trivedi. De Sitter vacua in string theory. Phys. Rev., D68:046005, 2003, hep-th/0301240.

[92] M. Kaku. Strings, conformal fields, and M-theory. New York, USA: Springer (2000) $531 \mathrm{p}$. 
[93] V. Kaplunovsky and J. Louis. Field dependent gauge couplings in locally supersymmetric effective quantum field theories. Nucl. Phys., B422:57-124, 1994, hepth/9402005.

[94] V. Kaplunovsky and J. Louis. On gauge couplings in string theory. Nucl. Phys., B444:191-244, 1995, hep-th/9502077.

[95] V. S. Kaplunovsky. One loop threshold effects in string unification. 1992, hepth/9205070.

[96] S. H. Katz, A. Klemm, and C. Vafa. Geometric engineering of quantum field theories. Nucl. Phys., B497:173-195, 1997, hep-th/9609239.

[97] S. H. Katz and C. Vafa. Geometric engineering of $\mathrm{N}=1$ quantum field theories. Nucl. Phys., B497:196-204, 1997, hep-th/9611090.

[98] V. V. Khoze, M. P. Mattis, and M. J. Slater. The instanton hunter's guide to supersymmetric SU(N) gauge theory. Nucl. Phys., B536:69-109, 1998, hep-th/9804009.

[99] E. Kiritsis. String theory in a nutshell. Princeton, USA: Univ. Pr. (2007) 588 p.

[100] E. Kiritsis and C. Kounnas. One Loop Corrections to Coupling Constants in IR-regulated String Theory. Nucl. Phys. Proc. Suppl., 45BC:207-216, 1996, hepth/9509017.

[101] B. Körs and P. Nath. Effective action and soft supersymmetry breaking for intersecting d-brane models. Nucl. Phys., B681:77-119, 2004, hep-th/0309167.

[102] D. Krefl. A gauge theory analog of some 'stringy' D-instantons. 2008, arXiv:0803.2829 [hep-th].

[103] M. Kuster, G. Raffelt, and B. Beltran. Axions: Theory, cosmology, and experimental searches. Proceedings, 1st Joint ILIAS-CERN-CAST axion training, Geneva, Switzerland, November 30-December 2, 2005.

[104] G. Lopes Cardoso and B. A. Ovrut. A green-schwarz mechanism for $\mathrm{d}=4, \mathrm{n}=1$ supergravity anomalies. Nucl. Phys., B369:351-372, 1992.

[105] J. Louis, T. Mohaupt, and S. Theisen. String theory: An overview. Lect. Notes Phys., 721:289-323, 2007.

[106] M. Luscher. Lattice QCD: From quark confinement to asymptotic freedom. Annales Henri Poincare, 4:S197-S210, 2003, hep-ph/0211220.

[107] D. Lüst. Lectures on monopoles and instantons. Munich U, Winter term 2006/07.

[108] D. Lüst. Intersecting brane worlds: A path to the standard model? Class. Quant. Grav., 21:S1399-1424, 2004, hep-th/0401156. 
[109] D. Lüst, P. Mayr, R. Richter, and S. Stieberger. Scattering of gauge, matter, and moduli fields from intersecting branes. Nucl. Phys., B696:205-250, 2004, hepth/0404134.

[110] D. Lüst and S. Stieberger. Gauge threshold corrections in intersecting brane world models. Fortsch. Phys., 55:427-465, 2007, hep-th/0302221.

[111] D. Lüst and S. Theisen. Lectures on string theory. Lect. Notes Phys., 346:1-346, 1989.

[112] V. Mukhanov. Physical foundations of cosmology. Cambridge, UK: Univ. Pr. (2005) $421 \mathrm{p}$.

[113] M. Nakahara. Geometry, topology and physics. Boca Raton, USA: Taylor \& Francis (2003) 573 p.

[114] M. Önder and A. Verçin. Orbits of the n-dimensional kepler-coulomb problem and universality of the kepler laws. European Journal of Physics, 27(1):49-55, 2006.

[115] R. D. Peccei and H. R. Quinn. CP Conservation in the Presence of Instantons. Phys. Rev. Lett., 38:1440-1443, 1977.

[116] M. Peskin. Instantons and nonperturbative QCD. Retrieved from the World Wide Web at http://www.slac.stanford.edu/ ${ }^{2}$ mpeskin/QFT.html.

[117] C. Petersson. Superpotentials From Stringy Instantons Without Orientifolds. 2007, arXiv:0711.1837 [hep-th].

[118] J. Polchinski. String theory. Vol. 1: An introduction to the bosonic string. Cambridge, UK: Univ. Pr. (1998) 402 p.

[119] J. Polchinski. String theory. Vol. 2: Superstring theory and beyond. Cambridge, UK: Univ. Pr. (1998) 531 p.

[120] J. Polchinski. Combinatorics of boundaries in string theory. Phys. Rev., D50:60416045, 1994, hep-th/9407031.

[121] J. Polchinski. Open heterotic strings. JHEP, 09:082, 2006, hep-th/0510033.

[122] J. Polchinski. The cosmological constant and the string landscape. 2006, hepth/0603249.

[123] R. Rajaraman. Solitons and Instantons. An Introduction to Solitons and Instantons in Quantum Field Theory. Amsterdam, Netherlands: North-Holland (1982) 409p.

[124] L. H. Ryder. Quantum Field Theory. Cambridge, Uk: Univ. Pr. (1985) 443p.

[125] A. N. Schellekens. The landscape 'avant la lettre'. 2006, physics/0604134.

[126] A. M. Semikhatov. Supersymmetric Instanton. JETP Lett., 35:560-563, 1982. 
[127] S. H. Shenker. The Strength of nonperturbative effects in string theory. Presented at the Cargese Workshop on Random Surfaces, Quantum Gravity and Strings, Cargese, France, May 28 - Jun 1, 1990.

[128] M. A. Shifman. Instantons in gauge theories. Singapore, Singapore: World Scientific (1994) $488 \mathrm{p}$.

[129] M. A. Shifman and A. I. Vainshtein. Solution of the anomaly puzzle in susy gauge theories and the wilson operator expansion. Nucl. Phys., B277:456, 1986.

[130] D. T. Son and A. O. Starinets. Viscosity, Black Holes, and Quantum Field Theory. Ann. Rev. Nucl. Part. Sci., 57:95-118, 2007, 0704.0240.

[131] D. N. Spergel et al. Wilkinson Microwave Anisotropy Probe (WMAP) three year results: Implications for cosmology. Astrophys. J. Suppl., 170:377, 2007, astro$\mathrm{ph} / 0603449$.

[132] L. Susskind. The anthropic landscape of string theory. 2003, hep-th/0302219.

[133] R. J. Szabo. An Introduction to String Theory and D-Brane Dynamics. Imperial College Press, 2004. ISBN 1-86094-427-2. 140p.

[134] G. 't Hooft. Computation of the quantum effects due to a four- dimensional pseudoparticle. Phys. Rev., D14:3432-3450, 1976.

[135] G. 't Hooft. Symmetry breaking through Bell-Jackiw anomalies. Phys. Rev. Lett., 37:8-11, 1976.

[136] G. 't Hooft. How Instantons Solve the U(1) Problem. Phys. Rept., 142:357-387, 1986.

[137] G. 't Hooft and M. J. G. Veltman. One loop divergencies in the theory of gravitation. Annales Poincare Phys. Theor., A20:69-94, 1974.

[138] M. Tegmark. On the dimensionality of spacetime. Class. Quant. Grav., 14:L69-L75, 1997, gr-qc/9702052.

[139] J. Terning. Modern supersymmetry: Dynamics and duality. Oxford, UK: Clarendon (2006) $324 \mathrm{p}$.

[140] A. I. Vainshtein, V. I. Zakharov, V. A. Novikov, and M. A. Shifman. ABC of instantons. Sov. Phys. Usp., 25:195, 1982.

[141] S. Vandoren and P. van Nieuwenhuizen. Lectures on instantons. 2008, arXiv:0802.1862 [hep-th].

[142] I. V. Volovich. Supersymmetric Yang-Mills Theories and Twistors. Phys. Lett., B129:429-431, 1983.

[143] S. Weinberg. The Quantum theory of fields. Vol. 1: Foundations. Cambridge, UK: Univ. Pr. (1995) 609 p. 
[144] S. Weinberg. The quantum theory of fields. Vol. 2: Modern applications. Cambridge, UK: Univ. Pr. (1996) 489 p.

[145] S. Weinberg. The U(1) Problem. Phys. Rev., D11:3583-3593, 1975.

[146] S. Weinberg. Anthropic Bound on the Cosmological Constant. Phys. Rev. Lett., 59:2607, 1987.

[147] F. Wilczek. Enlightenment, knowledge, ignorance, temptation. 2005, hep$\mathrm{ph} / 0512187$.

[148] E. Witten. Dynamical Breaking of Supersymmetry. Nucl. Phys., B188:513, 1981.

[149] E. Witten. Bound states of strings and p-branes. Nucl. Phys., B460:335-350, 1996, hep-th/9510135.

[150] E. Witten. Non-Perturbative Superpotentials In String Theory. Nucl. Phys., B474:343-360, 1996, hep-th/9604030.

[151] E. Witten. Reflections on the fate of space-time. Phys. Today, 49N4:24-30, 1996.

[152] E. Witten. World-sheet corrections via d-instantons. JHEP, 02:030, 2000, hepth/9907041.

[153] W. M. Yao et al. Review of particle physics. J. Phys., G33:1-1232, 2006.

[154] B. Zwiebach. A first course in string theory. Cambridge, UK: Univ. Pr. (2004) 558 p. 


\section{Lebenslauf}

Dipl.-Phys. James Nikolas Akerblom, verh. mit Heike Akerblom, geb. Scheibenzuber

12.11.1979 Geburt in Jönköping/Schweden

1986-1987 Besuch der Grundschule am Sandberge in Hannover

1987 Besuch der Calusa Elementary School in Boca Raton, Florida/USA

1987-1990 Besuch der Grundschule am Sandberge in Hannover

1990-1992 Besuch der Carl-Friedrich-Gauss-Schule in Hemmingen

1992-1999 Besuch der Humboldtschule Hannover, Abschluss 1999 mit dem Abitur

1999-2004 Studium der Physik an der Universität Hannover

2004 Abschluss an der Universität Hannover mit dem Diplom in Physik

seit 2005 Promotionsstudent in Physik an der Universität München

seit 2005 Angestellt als Promotionstudent am MPI für Physik in München 